\title{
O RESULTADO DE AX E KOCHEN \\ SOBRE UMA CONJECTURA DE ARTIN
}

STAVROS CHRISTODOULOU

DISSERTAÇÃO APRESENTADA AO

INSTITUTO DE MATEMÁTICA E ESTATÍSTICA

DA UNIVERSIDADE DE SÃO PAULO

PARA OBTENÇÃO DO GRAU DE MESTRE

EM MATEMÃTICA

ORIENTADOR: PROF. DR. ALFREDO ROSALIO JONES

SÃo PAULO, Setembro de 1978 


\section{PRE F $\vec{A} C I O$}

Sabe-se que em alguns corpos, se um polinômio homogêneo sobre o corpo tiver um nümero de variäveis suficientemente grande em relação ao seu grau, então ele admitiră automaticamente uma raiz não trivial no corpo.

Usa-se a nomenclatura $C_{i}(d)$ para designar um corpo no qual todo polinômio homogeneo de grau d em $n>d^{i}$ variáveis admite uma raiz não trivial. E diz-se que $K$ e e $C_{i}$ se for $C_{i}(d)$ para todo inteiro positivo d.

Chevaliey demonstrou que todo corpo finito $\vec{e} C_{1}$ (veja [Gr] cap.2). Juntando-se este resultado com o fato que se $K$ é $C_{i}$, então $K((X)$ ) ( o corpo das sêries formais com coeficientes em $K) \vec{e} C_{i+1}$, obtêm-se que os corpos $\mathbb{Z}_{p}((X))$ são $C_{2}$ para todo primo p (veja [Gr] teor.4.8 e corol.)

Artin conjecturou que os corpos dos nümeros p-ädicos $Q_{p}$-que são os completados de $Q$ com relação à valoração $p$ ädica (veja def. de valoração p-ädica na pag.8 e de $Q_{p} p g$. 45) - também seriam $C_{2}$; devido às semelhanças que estes corpos tem com os $\mathbb{Z}_{\mathrm{p}}((\mathrm{X}))$.

Sabe-se hoje que $Q_{p} \quad \bar{e} \quad C_{2}(2)$ (Hasse) e $C_{2}(3)$ (Demy anov para $p \neq 3$ e Lewis para todo $p$ ) para todo $p$; mas Terjanian apresentóu un exemplo de um polinômio homogeneo de grau 4 em 18 variảveis que não admite raizes não triviais em $Q_{2}$, mostrando assim que $Q_{2}$ não ê $C_{2}(4)$ (reproduzimos este ex. na $\$ 0$ do Cap.III). Posteriormente Schanuel mostrou que $Q_{p}$ não $\vec{e} \quad C_{2}(p(p-1))$ para todo primo impar p. (veja o cap.7 de [Gr] para todos estes resultados).

Ax e Kochen [A.K I] mostraram que dado d o conjunto $I(d)$ de todos os primos p tais que $Q_{p}$ não $\vec{e} \quad C_{2}(d) \quad \vec{e}$ fi nito (mostramos isto no corol.2 do teor.III 3.1). Este re- 
sultado e um caso particular de um resultado mais geral: que se A è uma förmula da linguagem de $1^{\mathrm{a}}$. ordem para cor pos valorados, então o conjunto dos primos $p$ tais que A vale em $\mathbb{Z}_{p}((X))$ e não vale em $Q_{p}$ e o conjunto dos pri mos $p$ tais que $A$ vale em $Q_{p}$ e não vale em $\mathbb{Z}_{p}((X))$ são ambos finitos (corol.1 do teor.III3.1). E este resulado é. consequência do fato que os ultraprodutos de $Q_{p}$ e $\mathbb{Z}_{p}((X))$ por qualquer ultrafiltro não principal do conjunto dos primos são isomorfos (teor.III3.1). E este fato segue de um teorema de isomorfismo entre corpos valorados que eles tambëm demonstraram (teor.II.2).

Neste trabaiho apresentamos uma introdução aos corpos valorados (no $1^{\circ}$ cap.; seguindo essencialmente [Rb] para as primeiras seções, e [Kp] e [Ko] para a 5a.); o teorema de $A x$ e Kochen (no 2 cap., seguindo [Ko]); e a aplicação à Conjectura de Artin (no $3^{\circ}$ ) . Lâ fazemos tambèm uma breve introdução à teoria dos modelos, uma vez que esta $\vec{a}$ a forma mais natural de trabalhar com linguagens formais e u1 traprodutos. 


\section{N D I C E}

CAPITULO I - UMA INTRODUÇÃO AOS CORPOS VALORADOS . ... 1

$\$ 1$ - Generalidades sobre corpos valorados . . . . . 1

$\$ 2$ - Comparação de valorações e construção de valora ções mais finas ............. 12

$\$ 3$ - Extensão de valorações . . . . . . . . 24

$\$ 4$ - Corpos valorados completos . . . . . . 35

$\$ 5$ - Corpos valorados pseudocompletos . . . . . 46

CAPITULO II - UM TEOREMA DE ISOMORFISMO ENTRE CORPOS VALORADOS . . . . . . . . . . . 73

CAPITULO III - O RESULTADO DE AX E KOCHEN SOBRE UMA CONJECTURA DE ARTIN •. . . . . . . . 95

$\S 0$ - Um contraexemplo à conjectura de Artin . . . 95

$\S 1$ - Uma introdução à teoria dos modelos . . . . 98

$\$ 2$ - U1traprodutos . . . . . . . . . . . 118

$\$ 3$ - 0 resultado de Ax e Kochen . . . . . . . 129

BIBLIOGRAFIA . . . . . . . . . . . . 146 
CAPITULO I

UMA INTRODUÇAO AOS CORPOS VALORADOS

\section{\$1. GENERALIDADES SOBRE CORPOS VALORADOS}

Nesta seção começamos definindo o que é uma valoração de um corpo, para logo a seguir introduzir os conceitos de anel e de corpo das classes residuais de uma valoração, que são fundamentais no estudo de corpos valorados. Finalizamos introduzindo mais algumas definições gerais:a de seção transversal de um corpo valorado (cross-section em Inglês), e de isomorfismo entre dois corpos valorados; e apresentando alguns exemplos de corpos valorados.

DEFINIÇÃO I1.1 - Seja K um corpo. Uma aplicação $v$ definida no conjunto $K^{*}$ dos elementos não nulos de $K$ e tomando valores num grupo aditivo ordenado $\Gamma$ se diz uma valoração de $K$, se:

(i) $v \vec{e}$ um homomorfismo do grupo multiplicativo $K^{*}$ no gru po aditivo $\Gamma$; isțo $\vec{e}$ se $a, b \in K^{*}$, então $v(a \cdot b)=v(a)+v(b)$.

(ii) se $a, b, a+b \in K^{*}$, então $v(a+b) \geq \min \{v(a), v(b)\}$ 
O grupo (abeliano) ordenado $G=v\left(K^{*}\right)$ - que escreveremos $v(K)$ para simplificar a notação - se diz grupo de valores de $K$ pela valoração $v$.

O par $\langle K, v\rangle-$ ou a tripla $\langle K, v, G\rangle$ conforme queiramos ou não realçar o grupo de valores G - se diz um corpo valorado. Convencionando $v(0)=\infty$ e $x<\infty, \quad x+\infty=\infty+x=\infty+\infty=\infty$ para todo $x \in G$, extendemos $v$ a todo $K$ de modo que as expressões de (i) e (ii) passam a valer para todos os elementos de K.

Vamos reunir num lema algumas consequências imediatas da definição, que serão frequentemente usadas nas manipulações com valorações:

LEMA I1.1 - Seja v uma valoração. Então:

(i) $v(1)=0, v\left(a^{-1}\right)=-v($ a $)$ para todo a $\neq 0$.

(ii) $v(-1)=0, v(-a)=v($ a) para todo $a \in K$.

(iii) Se $v(a) \neq t v(b)$, então $a+b \neq 0$ e $v(a+b)=$

$=\min \{v(a), v(b)\} \quad$ (e portanto $a+b=0$ ou $v(a+b)>$

$>\min \{v(a), v(b)\}$ implica que $v(a)=v(b))$.

(iv) $v\left(a_{1}+\ldots+a_{n}\right) \geq \min \left\{v\left(a_{1}\right), \ldots, v\left(a_{n}\right)\right\}$ e se existe

$i$ tal que $v\left(a_{i}\right)<v\left(a_{j}\right)$ para todo $j \neq i$, então

$a_{1}+\ldots+a_{n} \neq 0$ e $v\left(a_{1}+\ldots+a_{n}\right)=v\left(a_{i}\right)=\min \left\{v\left(a_{1}\right), \ldots\right.$

., v $\left.\left(a_{n}\right)\right\}$.

(e portanto $a_{1}+\ldots+a_{n}=0$ ou $v\left(a_{1}+\ldots+a_{n}\right)>$

$>\min \left\{v\left(a_{1}\right), \ldots, v\left(a_{n}\right)\right\}$ implica que existem $i \neq j$

tais que $\left.v\left(a_{i}\right)=v\left(a_{j}\right)\right)$. 
OBSERVAÇĀO: Estä sendo usado o 0 tanto para designar o elemento neutro do corpo K quanto o elemento neutro do grupo G, o que não deverả causar confusão.

\section{DEMONSTRAÇAO}

As conclusões de (i) são consequencias do fato de $v$ ser um homomorfismo de grupos.

Quanto a (ii) tem-se: $0=v(1)=v((-1) \cdot(-1))=v(-1)+$

$$
+v(-1) \text {. }
$$

Se $v(-1)<0$, então $v(-1)+v(-1)<0+0=0$ e se $v(-1)>0$, en tão $v(-1)+v(-1)>0+0=0, \operatorname{logo} v(-1)=0$, do que segue também que $v(-a)=v((-1) a)=v(-1)+v(a)=0+v(a)=v(a)$.

(iii) Seja $v($ a $) \neq v(b)$. Então é imediato, pelo item (ii), que $a+b \neq 0$ (se não $a=-b$ e $v(a)=v(-b)=v(b))$.

Sem prejuizo para a generalidade, seja $v(a)<v(b)$. Então: $v(a)=v((a+b)+(-b)) \geq \min \{v(a+b), \quad v(-b)\}=\min \{v(a+b), \quad v(b)\}=$ $=v(a+b)$ (não se pode ter $\min \{v(a+b), v(b)\}=v(b)$ senão $v(a) \geq v($ b) contra a suposição) .

Por outro lado $v(a+b) \geq \min \{v(a), v(b)\}=v(a)$. Juntando as desigualdades segue que: $v(a) \geq v(a+b) \geq v(a)$ e portanto $v(a+b)=v(a)=\min \{v(a), v(b)\}$.

(iv) A primeira parte deste item demonstra-se, por indu ção finita, a partir da condição (ii) da definição de valoração.

A segunda aplicando o item (iii) deste Lema com $a=a_{i}$ e 
$b=\left(a_{1}+\ldots+a_{n}\right)-a_{i}$ (e a primeira parte deste item para con cluir que $\left.v(b)=v\left(\sum_{j \neq i} a_{j}\right) \geq \min \left\{v\left(a_{j}\right): j \neq i\right\}>v\left(a_{i}\right)=v(a) \cdot\right)$.

PROPOSIÇAO I1.1 - Seja <K, v>um corpo valorado e sejam:

$\mathrm{R}_{\langle K, v>}=\{\mathrm{a} \in \mathrm{K}: \mathrm{v}(\mathrm{a}) \geq 0\}$ e $\mathrm{I}_{<\mathrm{K}, \mathrm{v}>}=\{\mathrm{a} \in \mathrm{K}: \mathrm{v}(\mathrm{a})>0\}$.

Então:

(i) $R_{\langle K ; v\rangle} \vec{e}$ um subanel unitärio de $K$, cujo corpo de frações é $K$.

(ii) $\mathrm{I}_{\langle K, v\rangle} \overrightarrow{\mathrm{e}} \mathrm{o}$ único ideal maximal de $\left.\mathrm{R}_{\langle K}, v\right\rangle$

\section{DEMONSTRAÇĀO}

(i) Sejam $a, b \in R<K, v>$. Então:

$v(a \pm b) \geq \min \{v(a), v( \pm b)\} \geq 0$ e $v(a \cdot b)=v(a)+v(b) \geq 0+0=0$, $\log 0 \mathrm{a} \pm \mathrm{b}, \mathrm{a} \cdot \mathrm{b} \in \mathrm{R}_{\langle K, v>}$, o que mostra que $\mathrm{R}_{<K}, v_{>} \overrightarrow{\mathrm{e}}$ um subane 1

de $K$. Também, como $v(1)=0$, segue que $1 \in R<K, v>$.

Seja agora $r \in K, r \notin R_{<} K, v>$. Então $v(r)<0$ e portanto $v\left(r^{-1}\right)=$ $=-v(r)>0$, donde $r-{ }^{1} \in R_{<K, v>}$ e comor $=\frac{1}{r-1}$ segue que $r$ es tá no corpo de frações de $R<K, v>$.

(ii) Sejam a, be $I_{<K, v>}$, então:

$v(a+b) \geq \min \{v(a), v(b)\}>0$, e portanto $a \pm b \in I<K, v>$

Se $a \in_{R}<K, v>$ e b€I $<K, v>$, então

$\mathrm{v}(\mathrm{a} \cdot \mathrm{b})=\mathrm{v}(\mathrm{a})+\mathrm{v}(\mathrm{b}) \geq 0+\mathrm{v}(\mathrm{b})>0+0=0$, e portanto

a.b€I $<K, v\rangle^{\cdot} \operatorname{Logo} I_{<K, v>} \vec{e}$ um ideal de $R_{<K, v>} \cdot$

o fato que $I_{\langle K, v\rangle} \vec{e}$ o ünico ideal maximal de $R_{\langle K, v\rangle}$ seguirả de (e é equivalente a): 
$\mathrm{R}_{\langle K, v>}>^{-\mathrm{I}}\langle\mathrm{K}, \mathrm{v}\rangle=\mathrm{U}\left(\mathrm{R}_{\langle\mathrm{K}, \mathrm{v}\rangle}\right):$ o conjunto dos elementos inversíveis de $R_{\langle K, v\rangle}$. Pois, uma vez que nenhum ideal pröprio pode con ter elementos inversíveis, todos os ideais próprios de $R_{<K, V>}$ estarão contidos em $R_{<K, v>}-U\left(R_{<K, V>}\right)=I_{<K, v>}$.

E de fato:

$a \in U\left(R_{\langle K, v>}\right) \Longleftrightarrow a \in R_{\langle K, v>}$ e $a^{1} \in R_{\langle K, v>} \Longleftrightarrow v(a) \geq 0$ e $v\left(a^{-1}\right) \geq$ $\geq 0 \Longleftrightarrow v(a) \geq 0$ e $v(a)=-v\left(a^{-1}\right) \geq 0 \Longleftrightarrow v(a)=0 \Longleftrightarrow v(a) \geq 0$ e nãc $v(a)>0 \Longleftrightarrow a \in R_{<K, v>}{ }^{e} a \in I_{<K, v>} \Leftrightarrow a \in R_{<K, v>}-I_{<K, v>}$

0 ane $1 R_{\langle K, v>} \vec{e}$ chamado anel da valoração $v$, o ideal $I_{K, v}>$ e chamado ideal da valoração $v$ e o corpo $\mathrm{R}_{\langle\mathrm{K}, \mathrm{v}\rangle} / \mathrm{I}_{\langle\mathrm{K}, \mathrm{v}\rangle}=$ $=\left\{a+I_{\langle K, v\rangle}: a \in R_{\langle K, v\rangle}\right\}$ (ê corpo uma vez que $I_{<K, v\rangle} \vec{e}$ ideal maximal de $R_{\langle K, v\rangle}$ ) $\vec{e}$ chamado corpo das classes residuais da valoração $v$, e serä denotado por $\vec{k}_{V}$.

Observe que em $\overline{\mathrm{K}}_{v}$ temos $\mathrm{a}=\mathrm{b}$ se e sö se $\mathrm{a}-\mathrm{b} \in \mathrm{I}_{<K, v>} \mathrm{e}$ portanto se e sö se $v(a-b)>0 ; e$ a $a b$ se e sö se $a-b \in$ $\mathrm{U}\left(\mathrm{R}_{\langle K, \mathrm{~K}\rangle}\right)$ e portanto se e só se $\mathrm{v}(\mathrm{a}-\mathrm{b})=0$. Em particular $\overline{\mathrm{a}}=$ $=\overline{0}$ se e só $\operatorname{se} v(a)>0$ e $a \not z 0$ se e sö se $v(a)=0$.

Vale também que se car $K=p \neq 0$, então car $K_{V}=p$,pois : $p \cdot 1=p\left(1+I_{\langle K, V\rangle}\right)=p \cdot 1+I_{\langle K, V\rangle}=0+I_{\langle K, V\rangle}=\overline{0}$ em $\bar{K}_{V}$, donde $\mathrm{p} \mid \operatorname{car} K_{\mathrm{V}}$ e portanto $\operatorname{car} \mathrm{K}_{\mathrm{V}}=\mathrm{p}$ (uma vez que $\overline{\mathrm{K}}_{\mathrm{V}} \neq\{0\}$. E que se car $\bar{K}_{V}=0$, então $\operatorname{car} K=0$ (consequência lögica da anterior); mas, como veremos pelos exemplos, $\vec{e}$ possivel que car $K=0$ e 
$\operatorname{car} K_{V}=p \neq 0$. Neste caso é claro que $v(p)>0$ e $v(n)=0$ para todo inteiro $1_{\leq} n<p$; do que segue que se $p \mid n$, então $v(n)=0$, pois: $n=q \cdot p+r$ com $o<r<p$ e portanto $v(r)=0$ enquanto que $v(p q)=v(p)+v(q) \geq v(p)+0>0$, donde pe1o Lema I1.1(iii) $v(n)=$ $=v(p q+r)=\min \{v(p q), v(r)\}=v(r)=0 ;$ e mais genericamente $v(n)=r \cdot v(p)$ se $n=p^{r} \cdot q$ com $p / q$

DEFINICAO II.2 - Seja $\langle K, v, G\rangle$ um corpo valorado. Uma aplica ção $\pi: G \longrightarrow K^{*}$ se diz uma seção transversal (ou $x$-seção) se i’ é um homomorfismo do grupo aditivo G no grupo multiplicativo $K^{*}$ tal que vom $: G \longrightarrow G$ e a identidade de $G$ - isto $\vec{e}:$ $\pi\left(g_{1}+g_{2}\right)=\pi\left(g_{1}\right) \cdot \pi\left(g_{2}\right)$ e $v(\pi(g))=g$ para todos $g_{1}, g_{2}, g \in G-$. As vezes denotaremos por $\pi^{g}$ o elemento $\pi(g)$, que é uma nota ção muito sugestiva para a igualdade: $\pi^{g_{1}+g_{2}}=\pi^{g_{1}} \cdot \pi^{g_{2}}$.

DEFINICAO II.3 - Sejam $\langle K, v, G\rangle$ e $\langle K, V, G\rangle$ dois corpos valora dos, e sejam $\phi: K \rightarrow K^{\prime}$ um isomorfismo de corpos entre $K$ e $K^{\prime}$ e $\mu: G \longrightarrow G^{\prime}$ um isomorfismo de grupos ordenados (isto $\vec{e}$ um isomorfismo de grupos que tambëm preserva a ordem) entre G e G'; tais que $\mu \circ v=V^{\prime} \circ \phi$ isto é: o seguinte diagrama é co mutativo:

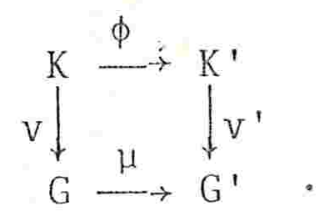


Nestas condições dizemos que a aplicação:

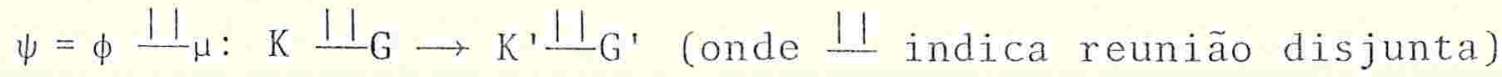
$\overrightarrow{\mathrm{e}}$ um isomorfismo de corpos valorados entre $\langle\mathrm{K}, \mathrm{v}, \mathrm{G}\rangle \mathrm{e}\left\langle\mathrm{K}^{\prime}, \mathrm{V}^{\prime}, \mathrm{G}^{\prime}\right\rangle$.

Esta è a definição geral que corresponde à definição de isomorfismo para estruturas similares (do mesmo tipo). Mas amiüde estaremos interessados mais nos isomorfismos entre os corpos $K_{\text {e }} K^{\prime}$, mesmo porque frequentemente teremos $G^{\prime}=\mathrm{G} . \mathrm{Pa}$ ra esta situação definiremos:

Um isomorfismo (algébrico) $\phi: K \longrightarrow \mathrm{K}^{\prime}$ se diz um isomorfismo analitico entre os corpos valorados $\langle\mathrm{K}, \mathrm{v}, \mathrm{G}\rangle$ e $\left\langle\mathrm{K}^{\prime}, \mathrm{v}^{\prime}, \mathrm{G}\right\rangle$ se $v=v^{\prime} \circ \phi$, isto é o seguinte diagrama é comutativo:

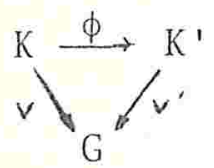

Se os corpos $\langle K, V, G\rangle$ e $\left\langle K^{\prime}, V^{\prime}, G\right\rangle$ admitirem $x$-seções $\pi$ e $\pi^{\prime}$, então um isomorfismo analïtico $\phi$ será dito um isomorfismo $x$-analitico se $\phi \circ \pi=\pi^{\prime}$, isto $\vec{e}$ o seguinte diagrama é comutativo:

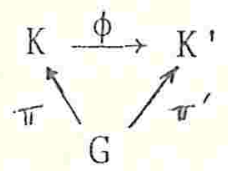


Vamos, para finalizar esta seção, ver alguns exemplos de corpos valorados.

$1^{\circ}\left\langle Q, v_{p}, \mathbb{Z}>\right.$ : Seja p um nümero inteiro primo. Dado $0 \neq \frac{a}{b} \in Q$ existe um ünico $r \in \mathbb{Z}$ tal que $\frac{a}{b}=p^{r} \frac{c}{d}$ com $p / c \cdot d$. Definindo $v_{p}(a / b)=r$ temos uma valoração do corpo dos racionais com grupo de valores $\mathbb{Z}$. Esta valoração chama-se valoração p-ädica dos racionais e demonstra-se que estas valorações (variando o primo p) são as ünicas valorações de Q com grupo de valores $\mathbb{Z}$.

0 ane $1 R_{\left\langle Q, v_{p}>\right.} \hat{e}\left\{\frac{a}{b} \epsilon Q: p \mid b\right\}$ e seu ideal maximal $I_{\left.<Q, v_{p}\right\rangle}=\left\{\frac{a}{b} \in Q: p \mid b\right.$ e p $\left.\mid a\right\}$. A aplicação:

$\phi: \mathrm{R}_{\left\langle\mathrm{Q}, v_{\mathrm{p}}>\right.} \rightarrow \mathbb{Z} / \mathrm{p} \cdot \mathbb{Z}=\mathbb{Z}_{\mathrm{p}}$ (onde $\mathbb{Z} / \mathrm{p} \cdot \mathbb{Z}$ e o corpo das classes de equivalência dos inteiros módulo p) definida por $\phi(a / b)=$ $=(\mathrm{a}+\mathrm{p} \cdot \mathbb{Z}) \cdot(\mathrm{b}+\mathrm{p} \cdot \mathbb{Z})^{-1}$ è um epimorfismo de anëis com núcleo igual a $I_{\left.<Q, v_{p}\right\rangle} \cdot \operatorname{Logo} \bar{Q}_{v_{p}}=R_{\left\langle Q, v_{p}\right\rangle} / I_{\left.<Q, v_{p}\right\rangle}=\mathbb{Z}_{p}$.

A aplicação $\pi: \mathbb{Z} \rightarrow$ Q definida por $\pi(n)=p^{n} \vec{e}$ uma $x-$ seção do corpo valorado $\left\langle\mathrm{Q}, \mathrm{v}_{\mathrm{p}}, \mathbb{Z}\right\rangle$.

$2^{\circ}\left\langle K(X), v_{p}, \mathbb{Z}\right\rangle$ : Seja $K$ um corpo, $K(X)$ seu corpo de fra ções racionais e p€K[X] um polinômio irredutîvel sobre $K$. Dado $0 \neq \frac{f}{g} \in K(X)$ existe um ünico $r \in \mathbb{Z}$ tal que $\frac{f}{g}=p^{r} \cdot \frac{f^{\prime}}{g}$, com $p \mid f^{\prime} \cdot g{ }^{\prime}$. Definindo $v_{p}(f / g)=r$ temos uma valoração de $K(x)$ com grupo de valores $\mathbb{Z}$ que é trivial em $K$. 
$R_{<K(x), v_{p}>}=\left\{\frac{f}{g} \in K(X): p \mid g\right\}$ e $I_{<K(x), v_{p}>}=\left\{\frac{f}{g} \in K(X): p|g, p| f\right\} \cdot D e-$ finindo $\phi: R_{<K(X), v_{p}>} \rightarrow K[X] / p \cdot K[X]=\{f+P \cdot K[X]: f \in K[X]\}$ por $\phi\left(\frac{\mathrm{f}}{\mathrm{g}}\right)=(\mathrm{f}+\mathrm{P} \cdot \mathrm{K}[\mathrm{X}]) \cdot(\mathrm{g}+\mathrm{p} \cdot \mathrm{K}[\mathrm{X}])^{-1}$, teremos um epimorfismo de anëis com nücleo $I_{<K(X), v_{p}>} \cdot$ E portanto:

$\overline{K(X)_{v}}=R_{<K}(X), v_{p}>/ I_{<K}(X), v_{p}>\approx K[X] / p \cdot K[X] \simeq K(\alpha)$ onde $\alpha \vec{e}$ uma raiz qualquer de $p$ (em alguma extensão de $K$ ).

$A$ aplicação $\pi: \mathbb{Z} \rightarrow K(x)$ definida por $\pi(n)=p^{n}$ e uma $x-$ se ção do corpo $<K(x), v_{p} \cdot \mathbb{Z}>$.

$3^{\circ}<\mathrm{K}(\mathrm{X}), \mathrm{V}_{\infty}, \mathbb{Z}>:$ Alêm das valorações dadas pelo exemplo ante rior $K(x)$ admite (apenas) mais uma valoração com grupo de va lores $\mathbb{Z}$ e trivial em $K$, definida por $v_{\infty}(f / g)=g r(g)-g r(f)$. $R_{\langle K(X), v>}=\left\{\frac{f}{g} \in K(X): \operatorname{grf} \leq \operatorname{grg}\right\}, I_{<K(X), v \infty\rangle}=\left\{\frac{f}{g} \in K(X): \operatorname{grf}<\operatorname{grg}\right\} . A$ aplicação $\left.\phi: R_{\langle K}(x), v_{\infty}\right\rangle \rightarrow K$ definida por $\phi\left(\frac{f}{g}\right)=\frac{a_{m}}{b_{n_{t}}}$ onde $m=\operatorname{grg}, \quad f=\sum_{i=0}^{n} a_{i} x^{i}, \quad g=\sum_{j=0}^{m} b_{j} x^{j} \quad\left(e a_{m}=0 \quad\right.$ se $\left.g x f<m\right) \vec{e}$ um epi morfismo de anëis com nücleo $I_{<K(X), v_{\infty}>} \cdot \operatorname{Logo} \overline{K(X)} v_{\infty} \simeq K$.

A aplicação $\pi: \mathbb{Z} \rightarrow K(X)$ definida por $\pi(n)=\left(\frac{1}{X}\right)^{n} \vec{e}$ uma $x-\sec \tilde{a} o$ de $<K(X), v,. \mathbb{Z}>$.

$4^{\circ}\langle K((X))$, ord, $\mathbb{Z}>$ : $K((X)) \vec{e}$ o corpo das séries formais com 
coeficientes em $K=\left\{\sum_{i=n}^{\infty} a_{i} x^{i}: n \in \mathbb{Z} ; a_{i} \in K\right\}$, e a valoração è defi nida por ord $\left(\sum_{i=n}^{\infty} a_{i} x^{i}\right)=n=\min \left\{i \in \mathbb{Z}: a_{i} \neq 0\right\}$

$\mathrm{R}_{\langle K((X:)), \text { ord }>}=\left\{\sum_{i=0}^{\infty} \mathrm{a}_{i} x^{i}: a_{i} \in K\right\}=K[[X]]$

$I_{<K}((X)), o r d>=\left\{\sum_{i=1}^{\infty} a_{i} x^{i}: a_{i} \in K\right\}$. A aplicação

$\phi: R_{<K}((X))$, ord $>\rightarrow K$ definida por $\phi\left(\sum_{i=0}^{\infty} a_{i} X^{i}\right)=a_{0} \vec{e}$ um ep $\underline{i}$ morfismo de anẹis com nücleo $\left.I_{<K(}(x)\right)$, ord $>$ de modo que: $K((X))_{\text {ord }} \simeq K$.

A aplicação $\pi: \mathbb{Z} \rightarrow K((X))$ definida por $\pi(n)=X^{n}$ eे uma $x-$ seção de $<K((X))$, ord, $\mathbb{Z}>$.

Observe que esta valoração è uma extensão da valoração $v_{x}$ de $K(X)$ do $2^{\circ}$ exemplo (com $\left.p=X \in K[X]\right)$, uma vez que se identifique $K(X)$ canonicamente como um subcorpo de $K((X))$.

5 $<K\left(\left(X^{g}\right)\right)$, ord, $G>$ : Este exemplo ê uma generalização do anterior para o caso em que G é um grupo abeliano ordenado qualquer. $K\left(\left(X^{g}\right)\right)$ é o corpo das sëries formais generalizadas com coeficientes em $\mathrm{K}$ e expoentes em G (conhecidas também como séries de potencias generalizadas de Hahn):

$K\left(\left(X^{g}\right)\right)=\left\{a=\sum_{\alpha \in G} a_{\alpha} X^{\alpha}: a_{\alpha} \in K\right.$ e $\sup (a)=\left\{\alpha \in G: a_{\alpha} \neq 0\right\} \hat{e}$ um conjunto 
bem ordenado\}.

A adição de duas tais séries se faz componente a componente e não oferece nenhum problema. A multiplicação tambêm se faz de forma usual: se $a=\sum a_{\alpha} x^{\alpha}, b=\sum b_{\beta} X^{\beta} \in K\left(\left(X^{g}\right)\right)$, então de$\alpha \in G \quad B \in G$

finimos $a b=\sum c_{\gamma} X^{\gamma}$ onde $c_{\gamma}=\sum a_{\alpha} b_{\beta}$. Só que agora temos que verificar se esta definição realmente é possível(isto é se para cada $\gamma \in G$ existe um número finito de pares $(\alpha, \beta)$ com $\alpha \in \sup (a), \beta \in \sup (b)$ tais que $\alpha+\beta=\gamma$, para que seja possíve1 fazer $\left(c_{\gamma}=\sum_{\alpha+\beta=\gamma} a_{\alpha} b_{\beta}\right)$ e se a sërie formal resultante de fato pertence a $K\left(\left(X^{g}\right)\right)$ isto è se $\sup (c)=\left\{\gamma \in G: c_{\gamma} \neq 0\right\}$ é bem ordenado:

Seja $\gamma \in G$; se existisse um número infinito de pares $(\alpha, \beta)$ com $\alpha \in \sup (a), \beta E \sup (b)$ tais que $\alpha+\beta=\gamma$, então poderíamos escolher uma sequência $\left(\alpha_{n}, \beta_{n}\right)$ entre estes pares tal que

$\alpha_{1}<\alpha_{2}<\ldots<\alpha_{n}<\ldots$ pois $\sup (a) \vec{e}$ bem ordenado. Como $\alpha_{n}+\beta_{n}=\gamma$, se guiria que $\beta_{1}>\beta_{2}>\ldots>\beta_{n}>\ldots$ contra a boa ordem de sup(b).

Para mostrar que sup(c) è bem ordenado é suficiente mos trar que $S=\{\alpha+\beta: \alpha \in \sup (a), \beta \in \sup (b)\} \vec{e}$ bem ordenado, pois $\sup (\mathrm{c}) \mathrm{C}$.

De fato se $S$ não fosse bem ordenado, existiria uma sequência $\gamma_{n}=\alpha_{n}+\beta_{n}$ de $S$ estritamente decrescente. Se existisse 
um nümero infinito de indices $n_{k}$ tais que $\alpha_{n_{k}}=\min \left\{\alpha_{n}: n \in N\right\}$, en tão a sequência $\beta_{n_{k}}$ seria estritamente decrescente contra a boa ordem de sup(b). Logo há um nümero finito de tais índices. Seja portanto $i_{0}=\max \left\{k \in N: \alpha_{k}=\min \left\{\alpha_{n}: n \in N\right\}\right\}$. Seguindo $o$ mesmo argumento podemosdefinir $i_{\ell+1}=\max \left\{k \in N: \alpha_{k}=\min \left\{\alpha_{n}: n>i_{\ell}\right\}\right\}$ e obter uma seqüência infinita $i_{0}<i_{1}<\ldots<i_{\ell}<\ldots$ de Indices tais que $\alpha_{i_{1}}<\alpha_{i_{2}}<\ldots<\alpha_{i_{\ell}}<\ldots$ donde $\beta_{i_{1}}>\beta_{i_{2}}>\ldots>\beta_{i_{l}}>\ldots$ contra a boa ordem de sup(b).

Com estas operações $K\left(\left(X^{g}\right)\right)$ è um corpo e definindo:

$\operatorname{ord}\left(\sum_{\alpha \in G} a_{\alpha} x^{\alpha}\right)=\min \left\{\alpha \in G: a_{\alpha} \neq 0\right\}$ temos que ord $\vec{e}$ uma valoraçãode $K\left(\left(X^{g}\right)\right)$ com grupo de valores $G$ e corpo das classes residuais isomorfo a $\mathrm{K}$.

\$2. COMPARAÇÃO DE VALORAÇÕES E CONSTRUÇÃO DE VALORAÇÕES MAIS FINAS

Nesta seção definiremos uma ordem entre valorações a par tir da relação de inclusão entre anéis de valoração e veremos como construir valoraçõescujo ideal seja um ideal primo dado do anel de alguma valoração. Isto se fará mostrando que existe uma correspondência bijetora entre os ideais primos do anel de uma valoração e os subgrupos convexos do grupo de valores daquela valoração, e associando então uma valoração 
a um subgrupo convexo dado. Finalmente aplicaremos esta construção aos corpos finitamente ramificados preparando-nos pa ra a situação que teremos no $2^{\circ}$ Capítulo.

LEMA 12.1 - Sejam $v$ e $v^{\prime}$ duas valorações de um corpo K. São equivalentes:

(i) $R_{\langle K, v\rangle}{ }^{\subset R}\left\langle K, v^{\prime}\right\rangle \quad$ (isțo é se $v(a) \geq 0$, então $v^{\prime}($ a $) \geq 0$ )

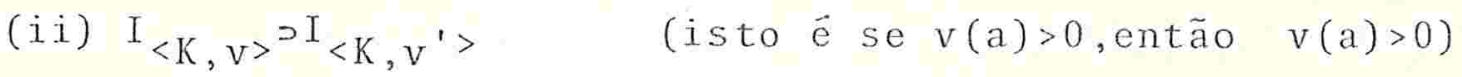

(iii) $U\left(R_{\langle K, v\rangle}\right) \subset U\left(R_{\left\langle K, v^{\prime}\right\rangle}\right)$ (isto é se $v(a)=0$, então $v^{\prime}(a)=0$ )

\section{Demonstração}

(i) $\Rightarrow$ (ii): Seja aEI $<K, v^{\prime}>$, isto $\overrightarrow{\mathrm{e}} v^{\prime}(\mathrm{a})>0$, então $v^{\prime}(1 / \mathrm{a})<0$

e por (i) (contrapositiva de i) $v(1 / a)<0$, donde $v(a)>0$, isto $\bar{e} \mathrm{a} \in I_{<K}, v>$

(ii) $\Rightarrow\left(\right.$ iii): Seja $a \in U\left(R_{\langle K, v>}\right)$, isto $\vec{e} v(a)=0$ e $v(1 / a)=0$. Se $v^{\prime}(a) \neq 0$, então $v^{\prime}(a)>0$ ou $v^{\prime}(1 / a)>0$ e por (ii) $v(a)>0$ ou $v(1 / a)>0 . \quad \log 0 v^{\prime}(a)=0$.

(iii) $\Rightarrow$ (i): Seja $a \in R_{<K, v>}$, isto $\vec{e} v(a) \geq 0$. Se $v(a)=0$, então por (iii) $v^{\prime}(a)=0$ e, portanto $a \in R_{<K, v>}$. Se $v(a)>0$, então pelo Le ma I1.1(iii) $v(1+a)=0$ e por (iii) $v^{\prime}(1+a)=0$, donde $v^{\prime}(a)=$ $=v^{\prime}((1+a)-1) \geq \min v^{\prime}(1+a), v^{\prime}(1)=0$ e portanto $\left.a \in R_{\langle K}, v^{\prime}\right\rangle \cdot$ Lo- 
go em qualquer caso se $a \in R_{\langle K}, v^{\prime}$, então $\left.a \in R_{\langle K}, v^{\prime}\right\rangle^{*}$

E imediato, a partir deste Lema, o seguinte:

COROLARIO - Sejam v e $v^{\prime}$ duas valorações de um corpo K. São equivalentes:

(i) $\mathrm{R}_{\langle K, v\rangle}=\mathrm{R}_{\left\langle K, \mathrm{~V}^{\prime}\right\rangle}$ (isto é v(a) 0 se e só se $v^{\prime}$ (a) 0)

(ii) $\mathrm{I}_{\langle\mathrm{K}, \mathrm{V}\rangle}=\mathrm{I}_{\left\langle\mathrm{K}, \mathrm{V}^{\prime}\right\rangle}$ (isto é v(a) 0 se e só se $v^{\prime}($ a) 0 ) $U\left(R_{<K, v\rangle}\right)=U\left(R_{\left\langle K, v^{\prime}\right\rangle}\right) \quad\left(\right.$ isto $\vec{e} v(a)=0$ se e só se $v^{\prime}(a)=0$ )

DEFINIÇAO I2.1 - Sejam v e V' duas valoraçōes de K.

(i) dizemos que $v$ é mais fina que $v^{\prime}$ e denotamos isto por $v \leq V^{\prime}$, quando $R_{\langle K, V}>^{R}\left\langle K, V^{\prime}\right\rangle^{\circ}$

(ii) dizemos que $v$ e $v^{\prime}$ não são comparáveis se não ocorre $v \leq v^{\prime}$ e nem $v \leq v$.

(iii) dizemos que $v$ e $v^{\prime}$ são equivalentes se $v \leq v^{\prime}$ e $v \leq v$ (isto $e$ quando $\left.R_{\langle K, v\rangle}=R_{\left\langle K, v^{\prime}\right\rangle}\right)$

$\vec{E}$ claro que a relação $\leq \vec{e}$ uma pre-ordem no conjunto das valorações (isto ê, ë reflexiva e transitiva); e a relação "ser equivalente" é de fato uma relação de equivalencia. Identificando as valorações equivalentes a relação $\leq$ induzido no quociente passa a ser uma ordem. 
E de fato duas valorações equivalentes são bastante parecidas, conforme pode se ver do seguinte resultado.

PROPOSIÇAO I2.1 - Duas valorações v e v' são equivalentes se e so se existe um isomorfismo de grupos ordenados $\mu$ entre $v(K)$ e $v^{\prime}(K)$, tal que $v^{\prime}=\mu \circ v$ (isto $\vec{e}$, comuta o diagrama

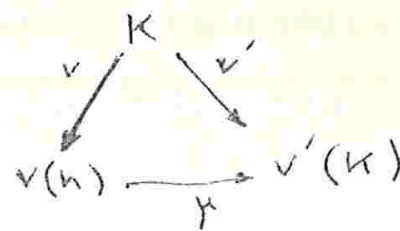

) .

\section{Demonstração}

Se existe um tal isomorfismo $\mu$ então $v(a) \geq 0$ se e só se $v^{\prime}(a)=\mu(v(a)) \geq \mu(0)=0$ e portanto $R_{\langle K, v\rangle}=R_{\left\langle K, v^{\prime}\right\rangle}$ o que mostra que $v$ e $v^{\prime}$ são equivalentes.

Reciprocamente se $v$ e $v^{\prime}$ são equivalentes e $v(a)=v(b)$, então $v(a / b)=v(a)-v(b)=0$ e portanto, pelo item (iii) do Corolário, $v^{\prime}(a / b)=0$, donde $v^{\prime}(a)=v^{\prime}(b)$.

Isto permite que se defina uma aplicação $\mu: v(K) \rightarrow v^{\prime}(K)$ que associa a $\alpha \in v(K), v^{\prime}(a) \in V^{\prime}(K)$ onde $a \in K$ e um elemento qualquer tal que $\alpha=v(a)$.

Se $\alpha=v(a)$ e $\beta=v(b)$, então $\mu(\alpha)=v^{\prime}(a), \mu(\beta)=v^{\prime}(b)$ e $v(a b)=v(a)+v(b)=\alpha+\beta$, donde $\mu(\alpha+\beta)=v^{\prime}(a b)=v^{\prime}(a)+v^{\prime}(b)=\mu(\alpha)+m(\beta)$, que mostra que $\mu$ é homomorfismo de grupos.

Se $0 \leqslant \alpha=v(a)$, entäo pelo item (ii) do Corolário, $0<v^{\prime}(a)=$ 
$=\mu(\alpha)$, o. que mostra que $\mu$ preserva ordem e é injetora.

Finalmente se $\alpha^{\prime} \in v^{\prime}(K)$, então existe a€K tal que $\alpha^{\prime}=v^{\prime}(a)=\mu(\alpha)$ onde $\alpha=v(a) \in v(K)$, o que mostra que $\mu$ é sobrejetora.

Se $I_{\left\langle K, v^{\prime}\right\rangle^{c I}\langle K, v\rangle}$ (isto $\left.\vec{e} v^{\prime} \leq v\right)$, então $I_{\left\langle K, V^{\prime}\right\rangle} \vec{e}$ um ideal primo de $R_{<K, v>}$. Estaremos interessados na situação contrâria: dado um ideal primo $\mathrm{P}$ de $\mathrm{R}_{\langle K, \mathrm{~K}, \mathrm{~V}}$, como construir (se pos sível) uma valoração $V^{\prime}$ tal que $\left.P=I_{\langle K}, v^{\prime}\right\rangle$. Para isto teremos que ver antes algumas definições em grupos ordenados.

DEFINIÇAO I2.2 - Un subgrupo H de um grupo abeliano ordenado G é um subgrupo convexo (ou isolado) de G, se gEG, hEH com $0<\mathrm{g} \leq \mathrm{h}$ implicam $\mathrm{g} \in \mathrm{H}$.

LEMA I2.2 - Sejam H um subgrupo convexo de G e geG.São equi valentes:

(i) $g>h$ para todo $h \in H$

(ii) $0<\mathrm{g}$ e $\mathrm{g} \notin \mathrm{H}$

(ii.i) $0<\mathrm{g}$ e $\overline{0}=0+\mathrm{H} \neq \mathrm{g}+\mathrm{H}=\overline{\mathrm{g}}$ em $\mathrm{G} / \mathrm{H}$.

\section{Demonstração}

(i) $\Rightarrow$ (ii): è óbvio

(ii) $\Leftrightarrow$ (iii): pela definição de G/H

(iii) $\Rightarrow$ (i): Se vale (ii) e $g \leq h$ para algum $h \in H$,então $0<\mathrm{g} \leq \mathrm{h}$ e portanto g pertenceria a $H$ por definição de subgrupo convexo. Logo g>h para todo hEH. 
PROPOSIÇÃO $12.2-$

(i) Se H è um subgrupo convexo de G, então G/H é um grupo ordenado pela relação $\bar{g}_{1}<\bar{g}_{2} \Leftrightarrow \bar{g}_{1} \bar{g}_{2}$ e $g_{1}<g_{2}$. A projeção ca nônica $\operatorname{pr}_{G, G / H}: G \rightarrow G / H$ é um epimorfismo de grupos ordenados.

(ii) Reciprocamente, se $\mu: G \rightarrow M$ è um homomorfismo de grupos ordenados, então $H=k$ er $\mu$ é um subgrupo convexo de G e G/H e Imu são isomorfos como grupos ordenados.

\section{Demonstração -}

(i) Precisamos inicialmente verificar que esta relação estä bem definida; isto ê se $\bar{g}_{1}^{\prime}=\bar{g}_{1}$ e $\bar{g}_{2}^{\prime}=\bar{g}_{2}$, então $g_{1}^{\prime}<g_{2}^{\prime}$. De fato, sejam $\bar{g}_{1}^{\prime}=\bar{g}_{1}$ e $\bar{g}_{2}^{\prime}=\bar{g}_{2}$, então $g_{1}^{\prime}=g_{1}+h_{1}$ e $g_{2}^{\prime}=g_{2}+h_{2}$ com $h_{1}, h_{2} \in H$ e $g_{2}^{\prime}-g_{1}^{\prime}=g_{2}-g_{1}+h_{2}-h_{1}=g_{2}-g_{1}+h$ com $h \in H$. Agora de $g_{1} \neq g_{2}$ e $g_{1}<g_{2}$ segue que $g_{2}-g_{1} \notin H$ e $0<g_{2}-g_{1}$ e pelo Lema an terior que $g_{2}-g_{1}>h$ para todo $h \in H$. Logo $g_{2}^{\prime}-g_{1}^{\prime}=g_{2}-g_{1}+h>0$ (uma vez que $g_{2}-g_{1}>-h \in H$ ).

A verificação de que esta relação é de ordem total e com pativel com a operação de $G / H$ e de que preserva a ordem de G se faz agora de modo natural.

(ii) Sejam gEG e $h \in H=$ ker $\mu$ tais que $0<g \leq h$. $\mu$ preserva a ordem, $\log 0$ :

$0=\mu(0) \leq \mu(g) \leq \mu(h)=0$ e portanto $\mu(g)=0$ e geker $\mu=H$. Isto mos tra que o subgrupo $\mathrm{H}$ é convexo e portanto G/H é um grupo or- 
denado e o isomorfismo de grupos entre G/N e Imu (que a $\bar{g}$ as socia $\mu(g) \in I m \mu$ ) também preserva a ordem, pois $\bar{g}_{1} \leq \bar{g}_{2} \Rightarrow$ $\Rightarrow g_{1} \leq g_{2} \Rightarrow \mu\left(g_{1}\right) \leq \mu\left(g_{2}\right)$.

LEMA I2.3 - Sejam <K, v, G>um corpo valorado.H um subgrupo con vexo de $G$ e $\operatorname{pr}_{G, G / H}: G \rightarrow G / N$ a projeção canônica. Então:

$V_{H}=p_{G, G / H}{ }^{\circ} \quad \vec{e}$ uma valoração de $K$ com grupo de valores $\mathrm{G} / \mathrm{H}$, anel de valoração $\mathrm{R}_{<\mathrm{K}, v_{\mathrm{H}}}=\{\mathrm{a} \in \mathrm{K}: v(\mathrm{a}) \Theta \mathrm{H}$ ou $v(\mathrm{a})>\mathrm{h}$ para to do $h \in H\}>R_{<K, v>}\left(\right.$ e portanto $\left.v_{H} \leq v\right), I_{<K, v_{H}>}=\{a \in K: v(a)>h$ para to do $h \in H\} \subset I_{<K, v>} e U\left(R_{<K, v_{H}>}\right)=\{a \in K: v(a) \in H\} \supset U\left(R_{<K, v>}\right)$.

\section{Demonstração -}

Pela definição $v_{H}(a)=\operatorname{pr}_{G, G / H}(v(a))=v(a)+H=\overline{v(a)} \in G / N \cdot C o-$ mo a projeção canonica $\operatorname{pr}_{G, G / H}: G \rightarrow G / H$ è um homomorfismo de grupos que preserva a ordem, tem-se:

$\overline{v(a \cdot b)}=\overline{v(a)+v(b)}=\overline{v(a)}+\overline{v(b)} \quad e$ $\overline{v(a+b)} \geq \overline{m i n}\{v(a), v(b)\}=\min \{\overline{v(a)}, \overline{v(b)}\}$ e portanto $v_{H} \vec{e}$ uma va loração de $K$ com grupo de valores G/H.

$a \in R_{<K, v_{H}>} \Longleftrightarrow v_{H}(a) \geq 0{ }_{G / H} \Longleftrightarrow \bar{v}(a) \geq \overline{0} \Longleftrightarrow \bar{v}(a)=\overline{0}$ ou $\overline{v(a)}>\overline{0}$ $\Longleftrightarrow v($ a $) E H$ ou $v(a)>h$ para todo $h \in H$.

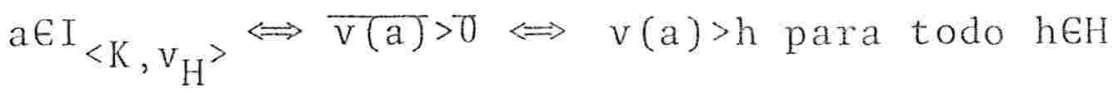

$$
\begin{aligned}
& \mathrm{a} \in U\left(\mathrm{R}_{<\mathrm{K}, \mathrm{v}_{\mathrm{H}}>}\right) \Longleftrightarrow \overline{v(\mathrm{a})}=\overline{0} \Longleftrightarrow v(\mathrm{a}) \in \mathrm{H}
\end{aligned}
$$


PROPOSIÇÃO I2.3 - Seja $\langle K, v, G\rangle$ um corpo valorado $\left.\mathrm{e}_{\varsubsetneqq} \mathrm{P} \mathrm{R}<\mathrm{K}, \mathrm{v}\right\rangle$ um ideal primo de $R_{<K, v>}$. Então o conjunto $H=\{h \in G:|h|<v(a)$ para todo aEP\} é um subgrupo convexo de $G$ e $P=I_{<K, V_{H}}$.

\section{Demonstração -}

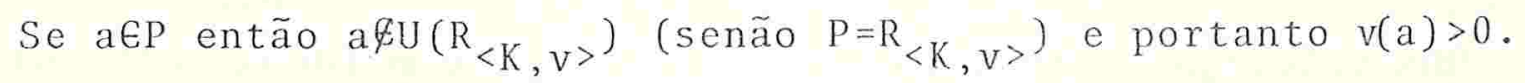
Logo $0 € H$.

, Sejam $h_{1}, h_{2} \in H$. Se $h_{1}-h_{2} \notin H$, então $\left|h_{1}\right|+\left|h_{2}\right| \geq\left|h_{1}-h_{2}\right| \geq v(a)$ para algum a€P.

Sejam $a_{1}, a_{2} \in K$ tais que $v\left(a_{1}\right)=\left|h_{1}\right|, v\left(a_{2}\right)=\left|h_{2}\right|$, então $v\left(a_{1} \cdot a_{2}\right)=v\left(a_{1}\right)+v\left(a_{2}\right)=\left|h_{1}\right|+\left|h_{2}\right| \geq v(a)$ e portanto $v\left(\frac{a_{1} \cdot a_{2}}{a}\right)=v\left(a_{1}\right)+v\left(a_{2}\right)-v(a) \geq 0$, donde $\frac{a_{1} \cdot a_{2}}{a}=r \in R R_{<K, v>}$ e $a_{1} \cdot a_{2}=r \cdot a \in P$ uma vez que $P$ é ideal.

De $v\left(a_{1}\right)=\left|h_{1}\right| \in H$ e $v\left(a_{2}\right)=\left|h_{2}\right| \in H$ segue que $a_{1} \notin A$ e $a_{2} \notin A$, o que contradiz o fato de ser $\mathrm{P}$ ideal primo de $\mathrm{R}_{<\mathrm{K}, \mathrm{V}\rangle}$; pois $a_{1}, a_{2} \in R_{<K, v>}$ (uma vez que $v\left(a_{1}\right)=\left|h_{1}\right| \geq 0$ e $v\left(a_{2}\right)=\left|h_{2}\right| \geq 0$ ) $\mathrm{a}_{1} \cdot \mathrm{a}_{2} \in \mathrm{P}$ mas $\mathrm{a}_{1} \notin \mathrm{P}$ e $\mathrm{a}_{2} \notin \mathrm{P}$.

Logo $h_{1}-h_{2} \in H$ e portanto $H$ è subgrupo de $G$.

Seja $0<\mathrm{g} \leq \mathrm{h} \in H$, então $|\mathrm{g}|=\mathrm{g} \leq \mathrm{h}=|\mathrm{h}|<\mathrm{V}(\mathrm{a})$ para todo a $\in \mathrm{P}$ e por tanto gEH, o que mostra que $H$ è subgrupo convexo de G. Se a€P e $h \in H$, então $v(a)>|h|, \operatorname{logo} v(a)$ satisfaz: $v(a)>h$ para todo $h \in H$ e portanto $a \in I_{<K, v_{H}>}$.

Reciprocamente se $a \in I_{<K, v_{H}}$, então $v(a)>h$ para todo $h \in H \quad e$ 
portanto $v(a) \geq v(b)$ para algum beP (senão $|v(a)|=v(a)<v(b)$ pa ra todo bEP e $v(a) \in H)$, donde $v\left(\frac{a}{b}\right) \geq 0$ e $\frac{a}{b}=r \in R_{<K, v>}$ e $a=r . b \in P$. $\mathrm{E}$ portanto $\mathrm{P}=\mathrm{I}<\mathrm{K}, \mathrm{V}_{\mathrm{H}}>^{\cdot}$

Vimos então que dado um ideal primo $\mathrm{P}$ de $\mathrm{R}_{\langle\mathrm{K}, \mathrm{V}\rangle}$ existe uma valoração mais fina que $v$ da qual $p \vec{e}$ o ideal. Pelo Corolário do Lema I1.2 e pela definição I1.2, todas as valora ções que tem por ideal P são equivalentes entre si.

E portanto estabelecemos que existem correspondências bi jetoras entre os ideais primos de $\mathrm{R}_{\langle\mathrm{K}, \mathrm{V}\rangle}$, os subgrupos convexos de $G=v(K)$ e as classes de equivalencia de valorações mais finas que $v$. Às vezes denota-se a valoração $v_{H}$ por $v_{p}$ onde $\mathrm{P}$ é o ideal que corresponde ao subgrupo H. (notação de $[\mathrm{Rb}]$; veja Caps. C e F).

Podemos ainda prosseguir e definir uma valoração no cor po $\bar{K}_{v_{H}}$ das classes residuais de $K$ pela valoração $v_{H}$. Isto se deve ao seguinte fato: Se $\overline{0} \neq \ddot{a}=a+I_{<K, v_{H}}=b+I_{<K, v_{H}>}=\bar{b} \in \bar{K}_{v_{H}}$, en tão $a \notin I_{<K, v_{H}>}$ e b-a€I $<K, v_{H^{\prime}}$, donde $v(a) \in H$ e $v(b-a)>h$ para todo hEH e portanto $v(a)<v(b-a)$ e pelo Lema I $1.1($ iii $) \quad v(b)=$ $=v(b-a+a)=\min \{v(b-a), v(a)\}=v(a) E H$. O qual mostra que pode mos definir uma aplicação $v / H(o u v / P)$ em $\bar{K}_{v_{H}}^{*}$ tal que se $\overline{0} \neq \bar{a}=a+I_{<K, v_{h}}>\in \bar{K}_{v_{H}^{*}}$, então $v / H\left(a+I_{<K, v_{H}}\right)=v(a) \in H$ (pois não depende do representante a). E facil de ver agora que $v / H$ e 
uma valoração de $\bar{K}_{V_{H}}$ com grupo de valores $H$.

LEMA I2.4 - o corpo $\left(\overline{\overline{\mathrm{K}}_{V_{H}}}\right)_{\mathrm{V} / \mathrm{H}}$ das classes residuais de $\overline{\mathrm{K}}_{V_{\mathrm{H}}}$ por $v / H$ e isomorfo a $\bar{K}_{V}$.

\section{DEMONSTRAÇÃO}

Vamos durante esta demonstração usar a notação $\bar{a}$ apenas para os elementos de $\bar{K}_{V_{H}}$ (isto è a $\in R_{<K, v_{H}>}$ e $\bar{a}=a+I_{<K, v_{H}>} \in \bar{K}_{V_{H}}$ ). Para os outros dois, $\overline{\mathrm{K}}_{V}$ e $\left(\overline{\overline{\mathrm{K}}_{V_{H}}}\right)_{V / H}$, escreveremos "explicitamente": $a+I_{\langle K, v>} \in \bar{K}_{V}$ onde $a \in R_{\langle K, v>} e \bar{a}+I_{<\bar{K}_{V_{H}}, v / H>} \in \bar{K}_{V_{H V / H}}$

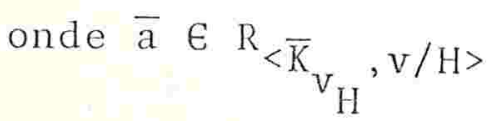

Sejam $\bar{a}+I_{<\bar{K}_{v_{H}}, v / H>}=\bar{b}+I_{<\bar{K}_{V_{H}}, v / H>} \in\left(\overline{\left(\bar{K}_{v_{H}}\right.}\right)_{v / H}$, então:

(i) $\overline{\mathrm{a}} \in \mathrm{R}_{\left\langle\overline{\mathrm{K}}_{v_{\mathrm{H}}}, \mathrm{v} / \mathrm{H}>\right.}$ e portanto $\mathrm{v} / \mathrm{H}(\overline{\mathrm{a}}) \geq 0$; mas $\mathrm{v} / \mathrm{H}(\overline{\mathrm{a}})=$ $=v(a) \geq 0$ e portanto a $\in R_{\langle K, v>}$. Analogamente $b \in R_{\langle K, v\rangle} e$ faz sentido $a+I_{\langle K, v\rangle}, b+I_{\langle K, v\rangle} \in \bar{K}_{V}$.

(ii) $\bar{b}-\bar{a} \in I_{<\bar{K}_{V_{H}}, v / H>}$ e portanto $v / H(\bar{b}-\bar{a})>0$, mas $v / H(\bar{b}-\bar{a})=v / H(\overline{b-a})=v(b-a)>0$ e portanto $b-a \in I_{<K, v>}$ $e \mathrm{~b}+\mathrm{I}_{<\mathrm{K}, \mathrm{v}\rangle}=\mathrm{a}+\mathrm{I}_{<\mathrm{K}, \mathrm{v}\rangle} \in \overline{\mathrm{K}}_{\mathrm{V}}$. 
Isto mostrou que é possível definir-se uma aplicação de
$\left(\overline{\mathrm{K}}_{V_{H}}\right)_{V / H}$ em $\bar{K}_{V}$ que a $\bar{a}+I_{\left.<\bar{K}_{V}, v / H\right\rangle}$ associa $a+I_{<K, v>}$ E é uma questão retineira agora verificar que esta aplicação é um isomorfismo entre os dois corpos.

Costuma-se dizer que v é composição das valorações $v_{H} e$ $\mathrm{v} / \mathrm{H}$.

Vamos agora ver esta construção aplicada à uma situação que ocorrerá no Cap. II:

DEFINICÃO I2.3 - Seja $\langle\mathrm{K}, \mathrm{V}, \mathrm{G}\rangle$ um corpo valorado. Definimos indice de ramificação (absoluto) de $\langle\mathrm{K}, \mathrm{v}, \mathrm{G}\rangle$ como sendo $\mathrm{e}=0$ se $\operatorname{car} \bar{K}_{V}=0$ e como sendo $e=$ cardinalidade do conjunto dos $x \in G$ tais que $0<x \leq v(p)=\{x \in G: 0<x \leq v(p)\}$ se $\operatorname{car} \bar{K}_{V}=p \neq 0$

Observe que se $\operatorname{cark}=p \neq 0$, então $v(p)=v(0)=\infty$ e $x<v(p)$ para todo $x \in G$, donde $\{x \in G: 0<x \leq v(p)\} \vec{e}$ inf $\underline{i}$ nito (pois não existe grupo ordenado, não trivial, finito). Logo se $\langle K, v, G\rangle$ é finitamente ramificado, então $\operatorname{carK}=0$. Se $\mathrm{e}=0$ ou e $=1$, dizemos que $\langle\mathrm{K}, \mathrm{v}, \mathrm{G}\rangle \overrightarrow{\mathrm{e}}$ não ramificado,

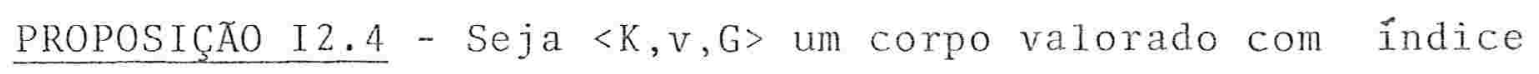
de ramificação e $>0$ e finito. Então existe $\min \{x \in G: 0<x\}=\gamma_{1}$, $\mathrm{v}(\mathrm{p})=\mathrm{e} \cdot \gamma_{1} \quad \mathrm{v}(\mathrm{n})=\mathrm{r} \cdot \mathrm{e} \cdot \gamma_{1}$ onde $0 \neq \mathrm{n}=\mathrm{p}^{\mathrm{r}} \cdot \mathrm{q} \quad \mathrm{com} \quad \mathrm{p} \not \mathrm{q} e$ 
$\{x \in G: 0<x \leq v(n)\} \vec{e}$ finito para todo $n \neq 0$ inteiro. 0 sub grupo $H=\mathbb{Z} \cdot \gamma_{1}$ de $G$, gerado por $\gamma_{1}$, é um subgrupo convexo de $\mathrm{G}$ e $\operatorname{car} \overline{\mathrm{K}}_{\mathrm{V}_{\mathrm{H}}}=0$.

\section{DEMONSTRAÇO}

O conjunto: $\{x \in G: 0<x \leq v(p)\}$ é finito e totalmente ordenado. Logo ele pode ser bem ordenado: $\left\{\gamma_{1}<\gamma_{2}<\ldots<\gamma_{e}=v(p)\right\}$.

E öbvio então que $\gamma_{1}$, o mínimo daquele conjunto, ê o mïnimo de $\{x \in G: 0<x\}$; bem como que cada $\gamma_{i+1}$ para $i=1, \ldots, e-1$, que o mínimo de $\left\{x \in G: \gamma_{i}<x \leq v(p)\right\}$, $\vec{e}$ o mínimo de $\left\{x \in G: \gamma_{i}<x\right\}$.

$\operatorname{Logo}$, como $\quad \gamma_{1}<\gamma_{1}+\gamma_{1}, \quad$ tem-se $\gamma_{2}=\min \left\{x \in G: \gamma_{1}<x\right\}<\gamma_{1}+\gamma_{1}$. Se $\gamma_{1}<\gamma_{2}<\gamma_{1}+\gamma_{1}$, então $0<\gamma_{2}-\gamma_{1}<\gamma_{1}$ contra a minimalidade de $\gamma_{1} \cdot \operatorname{Logo} \gamma_{2}=\gamma_{1}+\gamma_{1}=2 \gamma_{1}$. Analogamente e por indução $\gamma_{i}=$ i $\gamma_{1}$ pa ra $i=1, \ldots$, e. E portanto $v(p)=\gamma_{e}=e \gamma_{1}$.

Vamos agora mostrar que $\mathbb{Z}_{\gamma_{1}}$ e um subgrupo convexo de G:

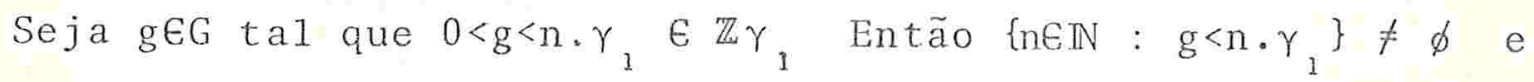
existe $m=\min \left\{n \in \mathbb{N}: g<n \gamma_{1}\right\}$. E claro que $m \geq 1$ e que $(m-1) \gamma_{1} \leq g$. $\operatorname{Logo}(m-1) \gamma_{1} \leq \mathrm{g}<m \cdot \gamma_{1}$ e $0 \leq \mathrm{g}-(\mathrm{m}-1) \gamma_{1}<\gamma_{1}-(m-1) \gamma_{1}=\gamma_{1}$ e pela minimalidade de $\gamma_{1}, 0=g-(m-1) \gamma_{1}$, ou $g=(m-1) \gamma_{1} \in \mathbb{Z}_{\gamma_{1}}$ o que mostra que $H=\mathbb{Z}_{\gamma_{1}} \vec{e}$ um subgrupo convexo de $G$.

Se $n$ è um inteiro (não nulo), então $v(n)=r \cdot v(p)$ onde $n=p^{r} \cdot q$ com $p \nmid q$ e portanto $v(n)=r \cdot e \cdot \gamma_{1} \in \mathbb{Z}_{\gamma_{1}} \cdot$ Se $0<x \leq v(n)$, 
então $x \in \mathbb{Z} \gamma_{1}$ (pois $\mathbb{Z}_{\gamma_{1}}$ é convexo) e $x=m \gamma_{1}$ com $0<m \leq r . e \cdot 1$ Logo $\{x \in G: 0<x \leq v(n)\}=\left\{m \gamma_{1}: 0<m \leq r . e\right\}$ tem $r . e$ elementos.

Finalmente, como $V(n)=r \cdot e \gamma_{1} \in \mathbb{Z}_{\gamma_{1}}=H$ (para $n \neq 0$ inteiro), segue pelo Lema I1.4 que $\left.n \in U\left(R_{<K}, v_{H}\right\rangle\right)$ e portanto $\overline{0} \neq \bar{n}=n \cdot \bar{I}$ em $\overline{\mathrm{K}}_{\mathrm{v}_{\mathrm{H}}}$, o que mostra que $\operatorname{car} \overline{\mathrm{K}}_{\mathrm{v}_{\mathrm{H}}}=0$.

\section{$\S 3$ EXTENST̃O DE VALORAÇÕES}

Seja $\langle K, v\rangle$ um corpo valorado e F um corpo que extende K. Krull mostrou (veja $[\mathrm{Rb}]$, Cap.8, Teor.5) que é sempre pos sivel extender $v$ a uma valoração $w$ de $F$.

Isto se faz extendendo $R_{\langle K, V\rangle}$ a um subanel de $F$ que pos sa ser anel de alguma valoração de F (existem condições necessarias e suficientes para que um subanel de um corpo seja o anel de alguma valoração deste corpo, veja por exemplo [Rb], Cap.8, Teor.1) e determinando então a partir do anel uma valoração.

No caso em que $F \vec{e}$ uma extensão transcendente simples, por exemplo $\mathrm{K}(\mathrm{X})$, temos uma grande liberdade de escolha para $w$, como se pode ver do seguinte resultado: seja $\Gamma$ um gru po ordenado que contêm $G=v(K)$ e $\gamma \in \Gamma(\gamma$ pode tambëm ou não pertencer a G). Então $W_{\gamma}$ definida por 
$w_{\gamma}(f)=\min \left\{v\left(a_{i}\right)+i \gamma: 0 \leqslant 1 \leqslant n\right\}$ onde $0 \neq f=\sum_{i=0}^{n} a_{i} x^{i} \in K[X] e^{\prime}$ $w_{\gamma}(f / g)=w_{\gamma}(f)-w_{\gamma}(g)$ para $f / g \in K(X)$ é uma valoração de $K(X)$ que extende $v([\mathrm{Rb}]$, Cap.8, Prop.5).

Quando a extensão é algébrica nã̃o temos mais esta 1jberdade e logo adiante veremos (Prop. I3.1) certas restricões que uma valoração de $F$ que extende $v$ deve satisfazer num ele mento algébrico sobre $K$.

Um caso de especial interesse ê quando a valoração $v$ do corpo valorado $\langle K, v\rangle$ admite um único prolongamento (a menos de equivalências) para cada extensão algébrica de K. Tais corpos são conhecidos como corpos henselianos (veja Rb, Cap.F, definição do corpo henseliano após Teor.2, e veja também o Corolário 2); e é notảvel de ver que esta propriedade do pro longamento único é equivalente a certos resultados sobre fatorabilidade de polinômios em $R_{<K, v>}[X]$, que além de serem propriedades intrínsecas, são definíveis em linguagem de $1^{\text {a }}$ ordem (veja o Cap. II para o conceito de linguagem de 1 a ordem), como por exemplo o Lema de Hensel:

Sejam $f, g, h \in R_{<K, v>}[X]$ tais que $\bar{f}=\bar{g} \cdot \bar{h}$ em $\bar{K} v$, comg e $h$ relativamente primos, $g r(\bar{g})=g r(g)>0$ e $g r(h) \leq g r(f)-g r(g)$. En tão existem $g^{*}, h^{*} \in R_{<K, v<}[X]$ tais que $f=g^{*} \cdot h^{*}, \bar{g}^{*}=\bar{g}, \bar{h}^{*}=\bar{h}$ e $\operatorname{gr}\left(g^{*}\right)=\operatorname{gr}(\mathrm{g}) \cdot($ Veja $[\mathrm{Rb}]$, Cap.F, Teor. 4). 
PROPOSIÇÃO I3.1 - Seja $\langle F, W>$ uma extensão de $\langle K, v>$ e $0 \neq$ a $\in F$ algëbrico sobre $K$. Entāo existe $0 \neq n \in \mathbb{Z}$ tal que n.w(a) $\in v(K)$.

\section{DEMONSTRAÇAO}

a algébrico sobre $K$ implica que existe $0 \neq f=\sum_{i=0}^{r} c_{i} X^{i} \in K[X]$ tal que $f(a)=\sum_{i=0}^{r} c_{i} a^{i}=0$. Pelo Lema II.1 (iv) existem $i \neq j$ tais que $w\left(c_{i} a^{i}\right)=w\left(c_{j} a^{j}\right)$, donde $w\left(c_{i}\right)+i \cdot w(a)=w\left(c_{j}\right)+j \cdot w(a) e$ portanto $(i-j) w(a)=w\left(c_{j}\right)-w\left(c_{i}\right)$.

Como $c_{i}, c_{j} \in K$ tem-se que $w\left(c_{i}\right)=v\left(c_{i}\right)$ e $w\left(c_{j}\right)=v\left(c_{j}\right)$ e portanto $w\left(c_{j}\right)-w\left(c_{i}\right)=v\left(c_{j}\right)-v\left(c_{i}\right) \in v(K)$ e como $i \neq j$, temse que $0 \neq n=i-j \in \mathbb{Z}$, ficando assim demonstrada a proposição.

Vamos agora, antes de apresentar algumas consequências des ta - simples porëm importante - proposição, introduzir algu mas definições e notações.

Se $K$ é um subcorpo de $F$ denotaremos por $\tilde{K}_{F}$ o fecho algébrico de $K$ em F, isto é o corpo de todos os elementos de F que são algébricos sobre $K$. Com esta notação K serä algébrica mente fechado em $F$ se e só se $K=\tilde{K}_{F}$; e F serâ uma extensão algëbrica de $K$ se e só se $\tilde{K}_{F}=F$.

Indicaremos por $\tilde{K}$ o fecho algébrico de $K$, isto é um corpo algêbricamente fechado que é uma extensão algêbrica de K. Sa- 
bemos da teoria dos corpos que não existe um ünico corpo com estas propriedades, mas que 2 tais corpos são sempre $K$-isomorfos; por isso identificaremos todos estes corpos entre si e falaremos no fecho algëbrico $\tilde{K}$ de $K$.

Recordamos também da teoria dos grupos que um grupo G se diz divisivel se dado $x \in G$ e $0 \neq n \in \mathbb{Z}$ existe y $\in G$ tal que $n \cdot y=x$.

Dado um grupo abeliano ordenado G; podemos construir um grupo abeliano ordenado e divisivel $\widetilde{G}$ contendo $G$, como sendo $\tilde{G}=\left\{\frac{g}{n}: g \in G\right.$ e $\left.0 \neq n \in \mathbb{Z}\right\}$ onde $\frac{g}{n}$ é a classe de equivalência de $(\mathrm{g}, \mathrm{n})$ pela relação $(\mathrm{g}, \mathrm{n}) \sim\left(\mathrm{g}^{\prime}, \mathrm{n}^{\prime}\right) \Leftrightarrow \mathrm{ng}^{\prime}=\mathrm{n}^{\prime} \mathrm{g}$ e $\frac{\mathrm{g}}{\mathrm{n}}+\frac{\mathrm{g}^{\prime}}{\mathrm{n}^{\prime}}=\frac{\text { r'g+ng' }^{\prime}}{\mathrm{n}^{\prime} \mathrm{n}^{\prime}}$ $\frac{g}{n} \leq \frac{g^{\prime}}{n^{\prime}} \Leftrightarrow n^{\prime} g \leq n^{\prime}$. Este grupo satisfaz o seguinte: dado um homomorfismo $\mu: G \rightarrow \Delta$ de grupos ordenados onde $\Delta$ é divisível, existe um ünico homomorfismo $\tilde{\mu}: \tilde{G} \rightarrow \Delta$ tal que $\mu=\tilde{\mu} \circ i$ onde $i$ $\vec{e}$ a inclusão de $G$ em $\tilde{G}$; isto é o diagrama $G \_i$ mutativo.

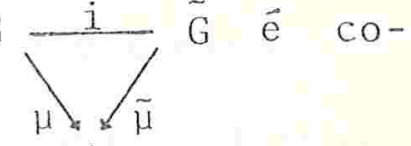

$\triangle$

Se G' também satisfaz esta propriedade de $\tilde{G}$, então existe um unico isomorfismo de grupos entre $\tilde{G}$ e $G^{\prime}$ que restrito a $G$ e a identidade, por isso identificaremos estes grupos entre si e diremos que $\tilde{G} \vec{e}$ o grupo divisivel gerado por G.

De acordo com estas notações a proposição I3.1 nos diz que se w è uma valoração de $\tilde{K}$ que extende $v$, então $w(\tilde{K}) \subset \widetilde{v(K)}$. Neste caso vale a igualdade $w(\tilde{K})=\widetilde{V(K)}$, pois, como é fácil 
ide ver, o grupo de valores de um corpo algebricamente fecha do é sempre um grupo divisível.

Um subgrupo H de um grupo abeliano $G$ se diz um subgrupo puro de $G$ (ou que admite divisão $\mathrm{cm} G$ ) se $n . g \in H$ com $0 \neq n \in \mathbb{Z}$ e gEG, implicam gEH.

Indicaremos por $\tilde{H}_{G}$ o menor subgrupo puro de $G$ que contém 11 . Neste caso (em que $H$ jả é um subgrupo de G), $\breve{H}_{G} \vec{e}$ dado por: $\tilde{H}_{G}=\{g \in G: n \cdot g \in I$ para algum $0 \neq n \in \mathbb{Z}\}$ e a demonstra ção disto se faz simplesmente verificando que este conjunto è de fato um subgrupo puro de G que contêm $H$ e estä contido em todos os subgrupos puros de G que contém H. (todas estas verificações são imediatas).

Com estas notações a proposição I3.I afirma que se $\langle\mathrm{F}, \mathrm{W}\rangle$ é uma extensão de $\langle K, v\rangle$ então $w\left(\tilde{K}_{F}\right) \subset \underset{v(K)}{V(F(F)}$. Às vezes es creveremos $\tilde{V}(K)_{W(F)}$ ao invés de $\widetilde{V}_{(K)}(F)$ quando isto não causar confusões (como por exemplo quando não existir alguma valoração $\tilde{v}$ ). Neste caso não podemos garantir a igualdadade entre $W\left(\tilde{K}_{F}\right)$ e $\tilde{v}(K)_{W(F)}$ : por exemplo se $v(K)=\mathbb{Z}, \quad F=K(X)$ e $w=w_{\gamma} \operatorname{com} \gamma=\frac{1}{2}$ do exemplo no início da seção, então $\tilde{K}_{F}=K$ e $w_{\gamma}\left(\tilde{K}_{F}\right)=\mathbb{Z}$ enquanto que $\tilde{v}(K) w_{\gamma}(F)=\frac{1}{2} \cdot \mathbb{Z}=\left\{\frac{1}{2} n: n \in \mathbb{Z}\right\}$. Mais adiante daremos condições para a igualdade ser possivel.

COROLARIO 1. - Seja $<K, v, G>$ um corpo valorado, F uma exten- 
săo algëbrica de $K$ e $w_{1}$ e $w_{2}$ duas extensões equivalentes de v. Então $w_{1}=w_{2}$.

\section{DEMONSTRACÃO}

Pela proposição $I 2.1$ existe um isomorfismo $\mu: w_{1}(F) \rightarrow W_{2}(F)$ tal que $\mathrm{w}_{2}=\mu \mathrm{w}_{1}$. Como $\mathrm{w}_{1}$ e $\mathrm{w}_{2}$ extendem $v$ tem-se tambëm que $\mu \mid G=i d_{G}$ e como $F$ é extensão algébrica de $K$ segue da propo sição I3.1 e dos comentários que $w_{1}(F)$ e $w_{2}(F)$ estão contidos em $\ddot{G}$. Seja então $\gamma=\frac{g}{n} \in w_{1}(F)$ com $g \in G$ e $0 \neq^{t} n \in \mathbb{Z}, \quad n \gamma=g \in G$ e portanto $n \cdot \mu(\gamma)=\mu(n \gamma)=\mu(g)=g$, donde $\mu(\gamma)=\frac{g}{n} \in w_{2}(F)$ o que mostra que $w_{1}(F)=w_{2}(F)$ e $\mu=i d_{w_{1}}(F)$ e portanto $\mu \circ w_{1}=i d o w_{1}=w_{1}=w_{2}$.

COROLARRIO 2. - Seja <K, v, G> um corpo valorado com x-seção $\pi$ e E um subcorpo de $K$ tal que $\pi \mid v(E)$ é uma $x$-seção para o cor po valorado $\langle E, v| E, v(E)>$. Nestas condições $v\left(\tilde{E}_{K}\right)=\tilde{v}(E)_{G}{ }^{*}$

\section{DEMONSTRAÇAO}

E claro que v é uma extensão de $v \mid E$, logo pela proposi ção I 3.1 v( $\left.\tilde{\mathrm{E}}_{K}\right) \subset \tilde{v}(\mathrm{E})_{\mathrm{G}}$.

Quanto à outra inclusão observe inicialmente que $\pi \mid v(E)$ é uma $x$-seção de $\langle E, v| E, v(E)>$ equivale apenas a dizer que $\pi(v(E)) \subset E$. Seja agora $g \in \tilde{v}(E)_{G}$. Então existe $0 \neq n \in \mathbb{Z}$ tal que $n g \in v(E)$ e portanto $\pi^{n g} \in E$. Como $\pi^{n g}=\left(\pi^{g}\right)^{n} \in E$ segue que $\pi^{g}$ é algëbrico sobre E, isto $\vec{e} \pi^{g} \in \tilde{E}_{K} \cdot \operatorname{Mas} v\left(\pi^{g}\right)=g$ 
e portanto $g \in v\left(\tilde{E}_{K}\right)$ o que mostra que $\tilde{v}(E)_{G} \subset v\left(\tilde{E}_{K}\right)$.

COROLARIO 3. - Seja $\langle$ F, w $>$ uma extensão de $\langle K, v\rangle$ tal que v(K) é um subgrupo puro de $w(F)$ e seja a€F tal que w(a) Ev(K). En täo a $\vec{e}$ transcendente sobre $K$ e w $(f(a))=\min \left\{v\left(c_{i}\right)+i \cdot w(a): 0 \leq i \leq n\right\}$ para todo $0 \neq f=\sum_{i=0}^{n} c_{i} x^{i} \in K[X]$ e (portanto) $w(K(a))=\{\gamma+i \cdot w(a): \gamma \in v(K), i \in \mathbb{Z}\}=v(K) \oplus \mathbb{Z} w(a)$ e 0 subgrupo de $w(F)$ gerado por $v(K)$ e $w(a)$.

\section{DEMONSTRAÇAO}

Se a fosse algébrico sobre $K$, então pela proposição I3.1 $w(a) \in w\left(\widetilde{K}_{F}\right) \subset \tilde{v}(K)_{w(F)}=v(K)$ uma vez que $v(K) \vec{e}$ puro em w(F). Logo a $\vec{e}$ transcendente sobre $K$.

Sejam $\gamma_{1}+i_{1} w(a)=\gamma_{2}+i_{2} \cdot w(a) \operatorname{com} \gamma_{1}, \gamma_{2} \in v(K)$ e $i_{1}$, $i_{2} \in \mathbb{Z}$. Então $\left(i_{1}-i_{2}\right) \cdot w(a)=\gamma_{2}-\gamma_{1} \in v(K)$ e se $i_{1} \neq i_{2}$, então $w(a) \in \widetilde{v}(K)_{w(F)}=v(K)$. Logo $i_{1}=i_{2}$ e $\gamma_{1}=\gamma_{2}$. Isto mostra que a soma $v(K)+\mathbb{Z} \cdot w(a) \vec{e}$ direta e que se $i \neq j$ e $c_{i}, c_{j} \in K$ não são nulos, entäo $v\left(c_{i}\right)+i \cdot w(a) \frac{t}{t} v\left(c_{n}\right)+j \cdot w(a)$, o que per mite aplicar-se o Lema 11.1 (iv) à $\sum_{i=0}^{n} c_{i} a^{i}$ para obter-se que $w\left(\sum_{i=0}^{n} c_{i} a^{i}\right)=\min \left\{v\left(c_{i}\right)+i \cdot w(a): 0 \leq i \leq n\right\}$ (pois uma vez que $w\left(c_{i} a^{i}\right)=v\left(c_{i}\right)+i \cdot w(a) \neq v\left(c_{j}\right)+j \cdot w(a)=w\left(c_{j}{ }^{j}\right)$, deve existir um indice $i$ tal que $w\left(c_{i} a^{i}\right){ }_{w}\left(c_{j} a^{j}\right)$ para todo $\left.j \neq i\right)$.

E desta expressão segue imediatamente que: 
$w(K(A))=\{\gamma+i \cdot w(a): \gamma \in v(K), i \in \mathbb{Z}\}=V(K) \oplus \mathbb{Z} \cdot w(a)$, e este $\vec{e}$ precisamente o subgrupo de w(F) gerado por $v(K)$ e w(a) .

COROLARIO 4. - Sejam <K, v, G> um corpo valorado, E um subcorpo de $K$ tal que $\pi^{V(E)} \subset E$ e $\beta E G$ - $v(E)$ tal que existe um inteiro primo $p$ e $p \cdot \beta \in v(E)$. Então $X^{p}-\pi^{p \beta} \in E[X]$ e o polinômio minimal de $\pi^{\beta}$ sobre $E$, (donde) $E\left(\pi^{\beta}\right)=\left\{f\left(\pi^{\beta}\right): \operatorname{fEE}[X]\right.$, grf $\left.<\mathrm{p}\right\}$.

Se $0 \neq f=\sum_{i=0}^{r} a_{i} X^{i} \in E[X]$ com $r p$, então

$v\left(f\left(\pi^{\beta}\right)\right)=v\left(\sum_{i=0}^{r} a_{i} \pi^{i \beta}\right)=\min \left\{v\left(a_{i}\right)+i \cdot \beta: 0 \leq i \leq r\right\}$ e (portanto) $v\left(E\left(\pi^{\beta}\right)\right)=\{\gamma+i \cdot \beta: \gamma \in v(E), 0 \leqslant i<p\}$ e o subgrupo de G gera-

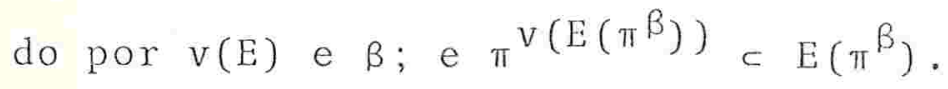

\section{DEMONSTRAÇAO}

Se $x^{p}-\pi^{p \beta}$ não fosse o polinômio minimal de $\pi^{\beta}$ sobre E, então existiria $0 \neq f=\sum_{i=0}^{n} a_{i} x^{i} \in E[X]$ com $r<p$ e $f\left(\pi^{\beta}\right)=0$ e em consequência do lema $I 1.1$ (iv) existiriam $0 \leq i \neq j \leq r \quad$ com $a_{i} \neq 0, a_{j} \neq 0$ e tais que $v\left(a_{i} \pi^{i \beta}\right)=v\left(a_{j} \pi^{j \beta}\right)$, donde $(i-j) \cdot \beta=$ $=v\left(a_{j}\right)-v\left(a_{i}\right) \in v(E)$. Mas $i-j$ é primo comp (pois $0<|i-j| \leq r<p$ e pé primo), logo existem $r, s \in \mathbb{Z}$ tais que $r(i-j)+s . p=1$, donde $\beta=1 \cdot \beta=r \cdot(i-j) \beta+s \cdot p \cdot \beta \in v(E)$ contra a hipótese.

Pelo mesmo argumento, dado $0 \neq f=\sum_{i=0}^{r} a_{i} X^{i} \in E[X]$ com $r<p$, $v\left(a_{i} \pi^{i \beta}\right) \neq v\left(a_{j}{ }^{j \beta}\right)$ sempre que $0 \leq i \neq j \leq p$ e $a_{i}$ e $a_{j}$ não são am- 
bos nulos. E portanto, em consequência do lema Il.1 (iv), $v\left(f\left(\pi^{\beta}\right)\right)=v\left(\sum_{i=0}^{r} a_{i} \pi^{i \beta}\right)=\min \left\{v\left(a_{i}\right)+i \cdot \beta: 0 \leq i \leq r\right\}$ e disto seguem imediatamente as afirmações que restam deste Corolârio.

COROLARIO 5. - Seja $\langle K, v>$ um corpo valorado e L um subcorpo de $K$ contido em $R_{\langle K, v\rangle}$. Então $v$ é trivial em toda extensão algëbrica de L em K (isto é se aft0, $v(a)=0$ ).

\section{DEMONSTRAÇAO}

Todo elemento não nulo de $L$ está em $R_{<K, v>}$ e admite um inverso em $L$ que portanto também está em $R_{<K}, v>$. Isto mostra que $L^{*} \subset U\left(R_{<K, v>}\right)$ : o conjunto dos inversíveis de $R_{<K, V>}$ e conforme se viu na demonstração da proposição Il.1 (ii) v é trivial em $U\left(R_{<K, V>}\right)$.

Se $0 \neq a \in K$ è algébrico sobre L, então pela proposição I3.1 existe $0 \neq n \in \mathbb{Z}$ tal que $n \cdot v(a) \in v(L)=\{0\}$, donde $n \cdot v(a)=0$ e portanto $v(a)=0$.

o interesse em subcorpos de $R_{<K, v>}$ se deve ao fato de que a projeção de $L$ em $\bar{K}_{v}$ é um monomorfismo (uma vez que é um homomorfismo entre corpos e como $L \neq\{0\}$ o homomorfismo é claramente não trivial). E se esta projeção for sobre, então teremos um subcorpo de $K$ isomorfo a $\bar{K}_{V}$ e teremos portan 
to um conjunto de representantes para as classes de $\overline{\mathrm{K}}_{V}$ num corpo. Nos exemplos $3,4,5$ da seção 1 . de fato $\bar{K}_{v}$ é isomorfo a um subcorpo de $K$.

E claro que para que L tenha alguma oportunidade de ser isomorfo a $\overline{\mathrm{K}}_{\mathrm{V}}$ ele deve ser pelo menos maximal em $\mathrm{R}_{<\mathrm{K}, \mathrm{v}>}$, i. to é não devem existir subcorpos de $K$ contidos em $R_{<K, v>}$ e contendo propriamente L.

PROPOSIÇAO I3.2 - Seja L um subcorpo maximal de $R_{K, v}$. Então:

(i) L é algebricamente fechado em K

(ii) $\bar{K}_{V}$ é uma extensão algébrica de $\bar{L}$.

\section{DEMONSTRACAO}

(i) Pelo Corolários da proposição I3.1 v é trivial em $\tilde{\mathrm{L}}_{\mathrm{K}}$ e portanto $\tilde{\mathrm{L}}_{\mathrm{K}}^{*} \subset U\left(\mathrm{R}_{<K, v>}\right)\left(\right.$ pois $\left.U\left(R_{<K, v>}\right)=\{\mathrm{a} \in K: v(a)=0\}\right)$. Logo $L \subset \tilde{L}_{K} \subset R_{\langle K, v>}$ e pela maximalidade de $L$ em $R_{<K, v>}$ segue que $L=\tilde{L}_{K}$.

(ii) Seja $\alpha \in \bar{K}_{V}$ e seja $a \in R_{<K, V>}$ tal que $\alpha=\bar{a}=a+I_{<K, V>}$. Se a€L, então $\alpha \in \bar{L}$ e portanto $\alpha$ é algêbrico sobre $\bar{L}$.

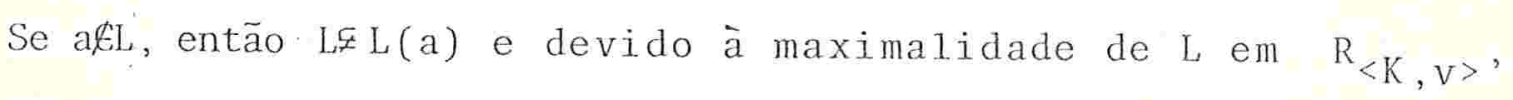
$L$ (a) não está contido em $\mathrm{R}_{<K, v>}$.

Existem então $f, g \in L[X], g \neq 0$ tais que $\frac{f(a)}{g(a)} \notin R_{<K, v>}$, isto é $v(f(a) / g(a))<0$. 
Daí segue que $v(g(a))>v(f(a)) \geq 0 \quad\left(v(f(a)) \geq 0\right.$ pois $f(a) \in R_{\langle K, V>}$ una vez que $\left.a \in R_{\langle K, v>} e f \in L[X] \quad R_{<K, v>}[X]\right)$ e portanto $g\left(\right.$ a) $\in I_{<K, v>}$, donde:

$\overline{0}=0+I_{\langle K, v\rangle}=g(a)+I_{\langle K, v\rangle}=\overline{g(a)}=\bar{g}(\bar{a})=\bar{g}(\alpha)$.

gEL[X] é um polinômio não nulo e portanto $\bar{g} \in \bar{L}[X]$ tambëm será um polinômio não nulo (pois se $0 \neq c \in L$, então $v(c)=0$ e portanto $\overline{\mathrm{c}} \neq \overline{0}$ em $\overline{\mathrm{K}}_{\mathrm{V}}$ de modo que se algum dos coeficientes de g. é não nulo, então algum dos coeficientes de $\bar{g}$ tamhëm será não nulo). Foi então encontrado um polinômio $\bar{g} \in \bar{L}[X], \bar{g} \neq 0$ com $\bar{g}(\alpha)=\overline{0}$ o que mostra que $\alpha$ é algébrico sobre $\bar{L}$.

TEOREMA 13.1 - Seja $\langle K, v>$ um corpo valorado que satisfaz o Lema de Hensel, $\operatorname{car} \bar{K}_{V}=0$ e $L$ um subcorpo maximal de $R_{<K, v\rangle^{\circ}}$ Então $\bar{L}=\bar{K}_{V}$ e portanto $L$ é isomorfo a $\bar{K}_{V}$.

\section{DEMONSTRAÇAOO}

Seja $\alpha \in \bar{K}_{V}$, pela proposição anterior, a é algébrico sobre $\bar{L}$, seja então $\phi=\operatorname{irr}(\bar{L}, \alpha) \in \bar{L}[X]$ o polinômio minimal de $\alpha$ em $\bar{L}[X] . \phi=\bar{f}$ para algum $f \in L[X]$ e em $\bar{K}_{V}[X] \phi=(X-\alpha) \cdot \xi$, $\operatorname{com} x-\alpha$ e $\xi$ primos entre si, pois $\operatorname{como} \operatorname{car} \bar{K}_{V}=0$ e $\phi \bar{e}$ i. $\underline{r}$ redutível em $\bar{L}[X]$, $\phi$ não pode admitir raizes múltiplas, don de $\xi(\alpha) \neq \overline{0}, X-\alpha \not \xi$ e $\operatorname{mdc}(X-\alpha, \xi)=1$.

Pelo Lema de Hensel existem $g^{*}, h^{*} \in R_{<K, V}[X]$ tais que $\bar{g}^{*}=X-\alpha$ e $\operatorname{gr}\left(g^{*}\right)=1$. Donde $g^{*}=c_{0}+c_{1} X \operatorname{com} \bar{c}_{1}=\bar{I}, \bar{c}_{0}=-\alpha$. 


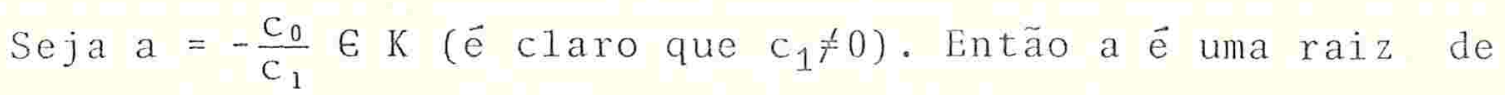
$g$ e a fortiori de $f$ e portanto a é algébrico sobre L, donde a EL pela proposição I 3.2 (i). Logo: $\alpha=-\overline{\mathrm{C}}_{0}=-\frac{\overline{\mathrm{C}}_{0}}{\overline{\mathrm{C}}_{1}}=\overline{\mathrm{a}} \in \overline{\mathrm{L}}$ o que mostra que $\bar{K}_{V} \subset \bar{L}$ e portanto $\bar{L}=\bar{K}_{V}$.

Observação: Se $\langle F, W\rangle$ e uma extensão de $\langle K, v\rangle$, então a aplicação que a $a+I_{\langle K, v>} \in \bar{K}_{V}$ associa $a+I_{\langle F, W\rangle} \in \bar{F}_{W}$ estä bem definida (pois se $a+I_{\langle K, V}=b+I_{\langle K, v>}$ em $\bar{K}_{v}$, então $v(a-b)>0, \quad w(a-b)>0$ e $\left.a+I_{<F, w>}=b+I_{<F, w>}\right)$ e $\vec{e}$ um monomorfismo.

Identificaremos $\bar{K}_{V}$ com sua imagem em $\overline{\mathrm{F}}_{\mathrm{W}}$ de modo que podemos considerar $\overline{\mathrm{K}}_{\mathrm{V}}$ como um subcorpo de $\overline{\mathrm{F}}_{\mathrm{W}}$.

Devido a esta identificação quando for dito, por exemplo, que $\bar{K}_{V}=\vec{F}_{W}$ isto significará que dado $a \in R_{<F, w>}$ existe $b_{\in} \in R_{<K, v>}$ tal que $w(b-a)>0$. Na prätica é suficiente encontrar b€K tal que $w(a-b)>0$, pois então seguirá forçosamente que $b \in R_{<K, v>}$.

\section{$\$ 4$ CORPOS VALORADOS COMPLETOS}

Se o grupo de valores de um corpo valorado $\langle K, v . G\rangle$ for um subgrupo - ou isomorfo a um subgrupo - de $\langle\mathrm{R},+, 0,<>$ (o grupo aditivo dos reais com a ordem usual)*, então a função $\mathrm{d}: \mathrm{K} \times \mathrm{K} \longrightarrow \mathbb{R}$ definida por: $\mathrm{d}(\mathrm{a}, \underline{\mathrm{b}})=\mathrm{e}^{-\mathrm{v}(\mathrm{a}-\mathrm{b})}$ se $\mathrm{a} \frac{\mathrm{t}}{\mathrm{t}} \mathrm{b}$ e $\mathrm{d}(\mathrm{a}, \mathrm{a})=0$,

(*) para isto $\vec{e}$ necessärio e suficiente que G seja arquimedeiano. 
satisfaz: $d(a, b) \geq 0$ e d (a,b) $=0 \Leftrightarrow a=b ; d(a, b)=d(b, a) \quad e$ $d(a, b)+d(b, c)=e^{-v(a-b)}+e^{-v(b-c)} \geq e^{-\min \{v(a-b), v(b-c)\}} \geq e^{-v(a-c)}=$ $=d(a, c) ;$ e portanto é uma métrica de $k$.

Nesta mëtrica vale: $a_{n} \rightarrow a \Leftrightarrow v\left(a_{n}-a\right) \rightarrow \infty$ em $\mathbb{R}$, pois $d\left(a_{n}, a\right)<\varepsilon \Leftrightarrow v\left(a_{n}-a\right)>\ln (1 / \varepsilon)$ e quando $\varepsilon$ fica arbitrariamente pequeno $\ln (1 / \varepsilon)$ torna-se arbitrariamente grande (e vi ce-versa). Tambëm $\left\{a_{n}\right\}_{n \in \mathbb{N}} \vec{e}$ de Cauchy $\Leftrightarrow v\left(a_{m}-a_{n}\right) \rightarrow \infty$ em $\mathbb{R}$ (quando $\mathrm{m}, \mathrm{n} \longrightarrow \infty$ ).

Outra propriedade é: se $a_{n} \rightarrow a \neq 0$, então existe $n_{0} \in \mathbb{N}$ tal que $v\left(a_{n}\right)=v(a)$ para todo $n \geq n_{0}$, pois tomando-se $n_{0} \in \mathbb{I N}$ tal que $v\left(a_{n}-a\right)>v(a)\left(f^{\infty}\right)$ para todo $n \geq n_{0}$, teremos (pelo Lema I1.1. (iii)) $v\left(a_{n}\right)=\min \left\{v\left(a_{n}-a\right), v(a)\right\}=v(a)$ para todo $n \geq n_{0}$. E um resultado anālogo vale se $\left\{a_{n}\right\} n \in I N$ é de Cauchy e $a_{n} \rightarrow 0$ : nes te caso existe $n_{0} \in \mathbb{N}$ tal que $v\left(a_{m}\right)=v\left(a_{n}\right)$ para todos $m, n \geq n_{0}$, pois de $a_{n} \rightarrow \rightarrow 0$ segue que $v\left(a_{n}\right) \rightarrow \infty$ e portanto existe $M>0$ tal que para todo $k \in \mathbb{N}$ existe $n \in \mathbb{N}$ tal que $v\left(a_{n}\right) \leq M$ e $n \geq k$. Por outro lado de $\left\{a_{n}\right\}_{n \in \mathbb{N}}$. de Cauchy segue que existe $n_{0} \in \mathbb{N}$ tal que $v\left(a_{m}-a_{n}\right)>M$ para todos $m, n \geq n_{0}$. Escolhendo $n \geq n_{0}$ tal que $v\left(a_{n}\right) \leq M$ teremos (pelo Lema I1.1 (iii)) que $v\left(a_{m}\right)=\min \left\{v\left(a_{m}-a_{n}\right)\right.$, $\left.v\left(a_{n}\right)\right\}=v\left(a_{n}\right)$ para todo $m \geq n_{0}$.

Se $\hat{K}$ é o completado de $\langle K, d\rangle$, então podemos definir $a+b=\lim _{n \rightarrow \infty}\left(a_{n}+b_{n}\right)$ e $a \cdot b=\lim _{n \rightarrow \infty}\left(a_{n} \cdot b_{n}\right)$ para elementos $a, b \in \widehat{K}$, 
onde $\left\{a_{n}\right\}_{n \in \mathbb{N}}$ e $\left\{b_{n}\right\}_{n \in N}$ são sequências de $K$ convergindo para a e b respectivamente. Com estas operações $\hat{K}$ torna-se um corpo. Podemos também definir $\hat{v}: \hat{K} \longrightarrow G$ por $\hat{v}(a)=v\left(a_{n}\right), \quad$ onde $\left\{a_{n}\right\}_{n \in \mathbb{N}}$ é uma sequência de $K$ que converge para a e $n$ é sufi cientemente grande no sentido de $v\left(a_{n}\right)=v\left(a_{m}\right)$ para todo $m>n$. $\langle\hat{K}, \hat{v}, G\rangle$ será então um corpo valorado que extende $\langle K, v, G\rangle$ e cujo grupo de valores é o mesmo de $\langle K, v, G\rangle$ conforme se vê pela definição de $\hat{v}$. Ainda mais o corpo das classes residuais $\overline{\widehat{K}}_{\hat{V}}$ de $\langle\hat{K}, \hat{V}, G\rangle$ e igual ao corpo das classes residuais $\bar{K}_{V}$ de $\langle K, V, G\rangle$; pois dado $\left.a \in R, \widehat{K}, \hat{v}\right\rangle$ sabemos que existe alguma sequência $\left\{a_{n}\right\}_{n \in \mathbb{N}}$ de $K$ convergindo para a e portanto $\hat{v}\left(a_{n}-a\right) \rightarrow \infty$, donde existe algum nEN tal que $\hat{v}\left(a_{n}-a\right)>0$ o que, de acordo com a observação no

Uma extensão de um corpo valorado que tem o mesmo grupo de valores e o mesmo corpo das classes residuais que o corpo se diz, de acordo com Krull, uma extensäo imediata do corpo dado.

Nós vimos então que $\langle\hat{\mathrm{K}}, \hat{\mathrm{v}}\rangle$ o completado de $\langle\mathrm{K}, \mathrm{v}\rangle$ ê uma extensão imediata de $<K, v>$.

Ainda de acordo com Krull um corpo valorado se diz maximalmente completo se não possui extensões imediatas prôprias. E claro então que un corpo valorado com grupo de valores arquimediano e maximalmente completo deverä ser completo (Cauchy-completo), umavez que seu completado, sendo-1he uma extensão imedia 
ta, terá que coincidir com ele.

No caso em que o grupo de valores é $\mathbb{Z}$ vale a recíproca: isto é se o corpo é completo, então ele é maximalmente completo (veja o início da próxima seção). Mas o melhor modo de estudar extensões imediatas e corpos maximalmente completos é através do conceito de pseudolimite, introduzido por Ostrowski, para corpos com grupos de valores quaisquer, como veremos na próxima seção.

Para o resto desta seção restringiremos ainda mais o gru po de valores para o caso em que ele é discreto e portanto i somorfo a $\mathbb{Z}$.

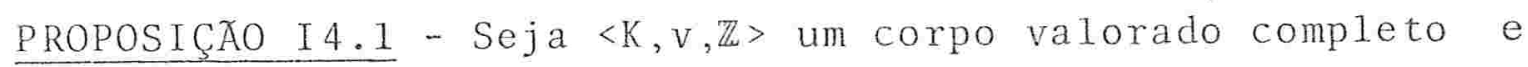
sejam $L \subset \dot{R}_{<K, v>}$ um conjunto completo de representantes para $\bar{K}_{V}$ com $O \in L$ (isto é se $a \in R_{<K, v}$ existe um unico beL talque $\bar{a}=\bar{b}$ em $\hat{K}_{v}$ ) e peK um elemento tal que $v(p)=1$.

Então todo a $\in K^{*}$ pode ser excrito como $a=\sum_{i=\ell}^{\infty} c_{i} p^{i}$, com $\ell \in \mathbb{Z}, c_{i} \in L$ e $c_{\ell} \neq 0 ;$ e este $\ell$ e os $c_{i}$-s satisfazendo estas condições são únicos.

E (reciprocamente) dado $\ell \in \mathbb{Z}$ e $c_{i} \in L$ para $i z \ell$ a somatória $\sum_{i=\ell}^{\infty} c_{i} p^{i}$ converge para algum elemento de $K$; de modo que pode mos escrever $K=\left\{\sum_{i=\ell}^{\infty} c_{i} p^{i}: \ell \in \mathbb{Z}, c_{i} \in L\right\}$. 


\section{DEMOSTRAÇĀO}

Seja $0 \neq a \in K$, então $v(a)=\ell \in \mathbb{Z}$ e $v\left({ }^{a} / p^{\ell}\right)=v(a)-v\left(p^{l}\right)=\ell-\ell \cdot 1=0$. Logo a/p $\mathrm{p}^{\ell} \in \mathrm{R}_{\langle K, V\rangle}$ e portanto existe $c_{\ell} \in L$ tal que $v\left(\frac{\mathrm{a}}{\mathrm{p}^{\ell}}-\mathrm{c}_{\ell}\right)>0$ ou $v\left(a-c_{l} p^{\ell}\right)>v\left(p^{l}\right)=\ell \quad\left(e ́\right.$ claro que $c_{\ell} \neq 0$, senão $\left.v\left(\frac{a}{p^{\ell}}-c_{\ell}\right)=v\left(\frac{a}{p^{\ell}}\right)=0\right)$.

Supondo escolhidos $c_{i} \in L$ para i de $\ell$ ate $n$, tais que $v\left(a-\sum_{i=\ell}^{n} c_{i} p^{i}\right)>n$, escolhe-se $c_{n+1} \in L$ do seguinte modo:

Se $v\left(a-\sum_{i=\ell}^{n} c_{i} p^{i}\right)>n+1$, então $c_{n+1}=0 \in L$.

Se $v\left(a-\sum_{i=\ell}^{n} c_{i} p^{i}\right)=n+1$, então $v\left(\frac{a-\sum_{i=\ell}^{n} c_{i} p^{i}}{p^{n+1}}\right)=(n+1)-(n+1)=0$ e portanto $\frac{\mathrm{a}-\sum_{i=\ell} c_{i} p^{i}}{p^{n+1}} \in R_{<K, v>}$ donde existe $c_{n+1} \in L$ tal que $v\left(\frac{a-\sum_{i}^{n} c_{i} p^{i}}{p^{n+1}}-c_{n+1}\right)>0$ ou $v\left(a-\sum_{i=\ell}^{n+1} c_{i} p^{i}\right)>v\left(p^{n+1}\right)=n+1$.

Em qualquer caso foi escolhido $c_{n+1} \in L$ tal que $v\left(a-\sum_{i=\ell}^{n+1} c_{i} p^{i}\right)>n+1$ de modo que a indução pode continuar. E a sequência $s_{n}=\sum_{i=\ell}^{n} c_{i} p^{i}$ converge para a, pois $v\left(a-s_{n}\right)=$ $=v\left(a-\sum_{i=\ell}^{n} c_{i} p^{i}\right)>n \rightarrow \infty$. Logo $a=\sum_{i=l}^{\infty} c_{i} p^{i}$ 
Se $a=\sum_{i=\ell}^{\infty} c_{i} p^{i}=\sum_{i=\ell}^{\infty}, c_{i}^{\prime} p^{i} \operatorname{com} c_{\ell} \neq 0 \neq c_{\ell}^{\prime}$, então $v(a)=v\left(\sum_{i=\ell}^{\infty} c_{i} p^{i}\right)=\ell \quad$ e $\quad v(a)=v\left(\sum_{i=\ell}^{\infty}, c_{i}^{\prime} p^{i}\right)=\ell$ ' segue $\ell=\ell^{\prime}$. $\log 0 \sum_{i=\ell+1}^{\infty} c_{i} p^{i}=\sum_{i=\ell}^{\infty} c_{i} p^{i}-c_{\ell} p^{\ell}=\sum_{i=\ell}^{\infty} c_{i}^{\prime} p^{i}-c_{\ell} p^{\ell}=\left(c_{\ell}^{\prime}-c_{\ell}\right) p^{\ell}+\sum_{i=\ell+1}^{\infty} c_{i}^{\prime} p^{i}$. Se $c_{\ell}^{\prime} \neq c_{\ell}$ então $c_{\ell}^{\prime}$ e $c_{\ell}$ estão em classes distintas de $K_{V}$

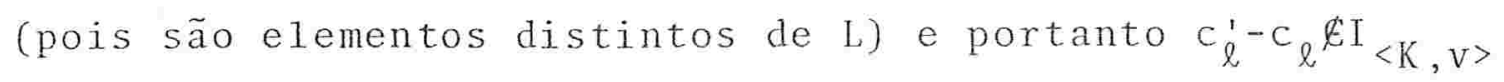
ou $v\left(c_{\ell}^{\prime}-c_{\ell}\right)=0$, donde $v\left(\left(c_{\ell}^{\prime}-c_{\ell}\right) p^{\ell}+\sum_{i=\ell+1}^{\infty} c_{i}^{\prime} p^{i}\right)=\ell \neq v\left(\sum_{i=\ell+1}^{\infty} c_{i} p^{i}\right) z \ell+1$. $\log 0 c_{\ell}^{\prime}=c_{\ell}$.

Supondo demonstrado que $c_{i}=c_{i}$ para $i=\ell, \ldots, n$, se $c_{n+1}^{\prime} \neq c_{n+1}$, então, pelo mesmo argumento anterior:

$v\left(\sum_{i=n+1}^{\infty} c_{i}^{i} p^{i}-c_{n+1} p^{n+1}\right)=n+1 \neq v\left(\sum_{i=n+1}^{\infty} c_{i} p^{i}-c_{n+1} p^{n+1}\right) \geq n+2 ;$ e portanto deve-se ter $c_{n+1}=c_{n+1}^{\prime}$. O que mostra a unicidade de $l$ e dos $c_{i}-S$.

(Reciprocamente) dados $c_{i} \in L$ para $i z \ell$ a sequência $s_{n}=\sum_{i=\ell}^{n} c_{i} p^{i}$ é de Cauchy, pois se $m>n$, então $v\left(s_{m}-s_{n}\right)=v\left(\sum_{i=n+1}^{m} c_{i} p^{i}\right)=\min \left\{i: n+1 \leq i \leq m \quad\right.$ e $\left.\quad c_{i} \neq 0\right\}>n+1 \quad$ ou $v\left(m^{-}{ }^{-}\right)=\infty$ se $c_{i}=0$ para todo $n+1 \leq i \leq m$. Em qualquer caso $v\left(m^{-}{ }_{n}\right)>\min \{m, n\} \rightarrow \infty$ quando $m, n \rightarrow \infty ;$ e como $<K, v, \mathbb{Z}>\vec{e}$ com pleto ela admite un 1 imite $a=\sum_{i=\ell}^{\infty} c_{i} p^{i}$. 
TEOREMA I4.1 (Hense1) Se $K, v, \mathbb{Z}$ é completo, então ele să tisfaz o Lema de Hensel.

DEMONSTRACAO

Sejam $f, g, h \in R_{\langle K, v>}[X]$ tais que $\bar{f}=\bar{g} \cdot \bar{h}$ em $\bar{K}_{v},(\bar{g}, \bar{h})=1$, $\operatorname{gr}(\bar{g})=\operatorname{gr}(g)=r>0 \quad$ e $\quad \operatorname{gr}(h) \leq \operatorname{gr}(f)-g r(g)=s$.

Como $(\bar{g}, \bar{h})=1$, então para cada $q=0,1, \ldots, r+s(=g r$ f $)$ e xistem $t_{q}, u_{q} \in R_{<K, v>}[X]$ tais que $g r\left(t_{q}\right) \leq s, \operatorname{gr}\left(u_{q}\right) \leq r \quad e$ $\bar{g}_{\mathrm{t}}+\overline{\mathrm{h}} \overline{\mathrm{u}}_{\mathrm{q}}=\mathrm{x}^{\mathrm{q}}$ (isto segue do Teorema de Bezout e do algorit mo da divisão euclideana vâlidos no anel $\bar{K}_{V}\left[X_{]}\right)$.

Vamos construir indutivamente uma sequência $\left.g_{n}, h_{n} \in K[X]\right)$ :

$\mathrm{g}_{0}=\mathrm{g}, \mathrm{h}_{0}=\mathrm{h}$

Construidos $g_{n}, h_{n} \in K[X]$ sejam:

$g_{n+1}=g_{n}+\sum_{q=0}^{r+s} p_{n, q} \cdot u_{q}, \quad h_{n+1}=h_{n}+\sum_{q=0}^{r+s} p_{n, q} t_{q}$, onde

$\sum_{q=0}^{r+s} p_{n, q} x^{q}=f-g_{n} h_{n}$.

Com relação a esta sequência valem:

1. $\mathrm{f}_{n}, \mathrm{~g}_{\mathrm{n}} \in \mathrm{R}_{<K, v>}[\mathrm{X}], \operatorname{gr}\left(\mathrm{g}_{\mathrm{n}}\right) \leq \mathrm{r}, \operatorname{gr}\left(\mathrm{h}_{\mathrm{n}}\right) \leq \mathrm{s}, \overline{\mathrm{g}}_{\mathrm{n}}=\overline{\mathrm{g}}_{\mathrm{g}}, \bar{h}_{n}=\overline{\mathrm{h}}$

2. Se $f-g_{n} h_{n} \neq 0$, então $\gamma_{n}<\gamma_{n+1}$, onde $\gamma_{n}=\min \left\{v\left(p_{n, q}\right): 0 \leq q \leq r+s\right\}$

Demonstração das afirmações:

1. para $n=0$ valem todas as afirmações pela escolha $g_{0}=g$, $h_{0}=h$. 


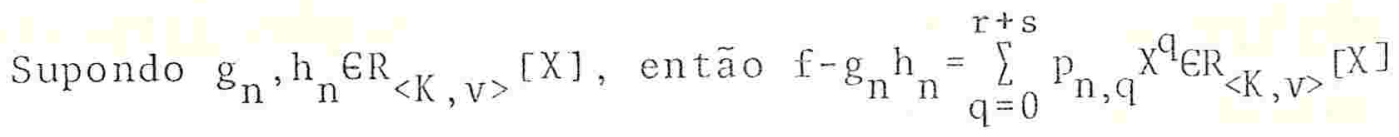
e portanto $p_{n, q} \in R_{\langle K, v>}$ donde $g_{n+1}, h_{n+1} \in R_{<K, v>}[X]$.

Supondo $\operatorname{gr}\left(g_{n}\right) \leq r, \operatorname{gr}\left(h_{n}\right) \leq s$ então $\operatorname{gr}\left(g_{n+1}\right) \leq \max \left\{g r\left(g_{n}\right), \operatorname{gr}\left(u_{q}\right): 0 \leq q \leq r+s\right\} \leq r$ $\operatorname{gr}\left(h_{n+1}\right) \leq \max \left\{\operatorname{gr}\left(h_{n}\right), \operatorname{gr}\left(t_{q}\right): 0 \leq q \leq r+s\right\} \leq s$

Supondo $\overline{\mathrm{g}}_{\mathrm{n}}=\overline{\mathrm{g}}$ e $\overline{\mathrm{h}}_{\mathrm{n}}=\overline{\mathrm{h}}$ então:

$\sum_{q=0}^{r+s} \bar{p}_{n, q} x^{q}=\overline{f-g_{n} h_{n}}=\bar{f}-\bar{g}_{n} \bar{h}_{n}=\bar{f}-\bar{g} \cdot \bar{h}=0$, donde $\bar{p}_{n, q}=\overline{0}$ e portanto $\bar{g}_{n+1}=\bar{g}_{n}+\sum_{q=0}^{r+s} \bar{p}_{n, q} \cdot \bar{u}_{q}=\bar{g}_{n}=\bar{g}_{c} \quad$ c analogamente $\bar{h}_{n+1}=\bar{h}_{n}=\bar{h}$.

2. Seja $f-g_{n} h_{n} \neq 0$, então $\gamma_{n}=\min \left\{v\left(p_{n, q}\right): 0 \leqslant q \leqslant r+s\right\}<\infty$

Seja $d_{n} \in K$ tal que $v\left(d_{n}\right)=\gamma_{n}$. Como $\bar{p}_{n, q}=\overline{0}$ segue que $v\left(p_{n, q}\right)>0$ e portanto $\gamma_{n}>0$ donde $d_{n} \in I_{<K, v>}$. Por outro lado como $v\left(p_{n, q} \geq \min \left\{v\left(p_{n, q}\right)\right\}=\gamma_{n}=v\left(d_{n}\right)\right.$ segue que $v\left(\frac{p_{n}, q}{d_{n}}\right) \geq 0$ e por tanto $p_{n, q} / d_{n} \in R_{<K, v>}$ ou $p_{n, q} \in d_{n} \cdot R_{<K, v>}$.

Também $\overline{x^{q}-g_{n} t_{q}-h_{n} u_{q}}=x^{q}-\overline{g_{n} t_{q}}-\overline{h_{n} \bar{u}_{q}}=x{ }^{q}-\bar{g} \bar{t}_{q}-\bar{h} \overline{u_{q}}=0$, donde $x^{q}-g_{n} t_{q}-h_{n} u_{q} \in I_{<K, v>}[X]$ e portanto existe $d \in I_{<K, v>}$ tal que $x^{q}-g_{n} t_{q}-h_{n} u_{q} \in d \cdot R_{<K, v>}[X]$.

Então:

$f-g_{n+1} h_{n+1}=f-\left(g_{n}+\sum_{q=0}^{r+s} p_{n, q} u_{q}\right) \cdot\left(h_{n}+\sum_{q=0}^{r+s} p_{n, q} t_{q}\right)=$ 
$=f-g_{n} h_{n}-g_{n} \cdot \sum_{q=0}^{r+s} p_{n}, q^{t} q-h_{n} \cdot \sum_{q=0}^{r+s} p_{n, q} u_{q}-$

$-\sum_{q=0}^{r+s} \sum_{q^{\prime}=0}^{r+s} p_{n, q^{n}} p_{n, q}, u_{q} q^{\prime}$. Mas:

$$
\begin{aligned}
& f-g_{n} h_{n}-g_{n} \cdot \sum_{q=0}^{r+s} p_{n, q} \tau_{q}-h_{n} \cdot \sum_{q=0}^{r+s} p_{n, q} u_{q}= \\
& \begin{array}{l}
=\sum_{q=0}^{r+s} p_{n, q}\left(x^{q}-g_{n} t_{q}-h_{n} u_{q}\right) \in d_{n} \cdot d \cdot R_{<K, v}[x] \\
\sum_{q}^{r+s} \sum_{q^{\prime}=0}^{r+s} p_{n, q^{p}} p_{n, q^{\prime}} u_{q} t^{\prime} q^{\prime} \in d_{n} \cdot d_{n} \cdot R_{<K, v>}[x]
\end{array}
\end{aligned}
$$

Seja $d_{n+1} \in K$ tal que $v\left(d_{n+1}\right)=\min \left\{v\left(d_{n} d\right), v\left(d_{n} d_{n}\right)\right\}$,

então $f-g_{n+1} h_{n+1} \in d_{n+1} \cdot R_{<k, v}[x] \quad$ (pois ambas as parcelas pertencerão a $\left.d_{n+1} \cdot R_{<K, v>}[X]\right)$. Como $f-g_{n+1} h_{n+1}=$ $\sum_{q=0}^{r+s} p_{n+1}, q^{x^{q}}, \quad$ segue então que $\gamma_{n+1}=\min \left\{v\left(p_{n+1, q}\right): 0 \leq q \leq r+s\right\} \geq v\left(d_{n+1}\right)$.

Mas $v\left(d_{n} d\right)=v\left(d_{n}\right)+v(d)>v\left(d_{n}\right)+0=\gamma_{n} \quad\left(\right.$ pois $\left.d \in I_{<K, v>}\right)$ e $v\left(d_{n} d_{n}\right)=v\left(d_{n}\right)+v\left(d_{n}\right)>v\left(d_{n}\right)+0=\gamma_{n} \quad\left(\right.$ pois $\left.d_{n} \in I_{<K, v>}\right)$ $\log 0 \gamma_{n+1} \geq v\left(d_{n+1}\right)=\min \left\{v\left(d_{n} d\right), v\left(d_{n} d_{n}\right)\right\}>\gamma_{n}$ (valem as desigualdades estritamente, pois $\gamma_{n}<\infty$ ).

Se para algum $n_{0} \in \mathbb{N}, \quad f-g_{n_{0}} h_{n_{0}}=0$, então $g_{n_{0}}, h_{n_{0}}$ satisfazem: $\quad \mathrm{f}=\mathrm{g}_{\mathrm{n}_{0}} \mathrm{~h}_{\mathrm{n}_{0}}, \quad \overline{\mathrm{g}_{\mathrm{n}_{0}}}=\overline{\mathrm{g}}, \quad \overline{\mathrm{h}_{\mathrm{n}_{0}}}=\overline{\mathrm{h}}$ e $\operatorname{gr}\left(\mathrm{g}_{\mathrm{n}_{0}}\right)=$ $g r(g)=r$ e estara assim satisfeito o Lema de Hensel.

Se não, a sequência $\left\{\gamma_{n}\right\}_{n \in \mathbb{N}}$ è estritamente crescente 
em $\mathbb{Z}$ e portanto $\gamma_{n}{ }^{+\infty} \cdot$ Sejam:

$g_{n}=\sum_{i=0}^{r} a_{n, i} x^{i} \quad$ e $\quad h_{n}=\sum_{j=0}^{s} b_{n, j} x^{j}$. Entäo:

$\sum_{i=0}^{r}\left(a_{n+1, i}-a_{n, i}\right) x^{i}=g_{n+1}-g_{n}=\sum_{q=0}^{r+s} p_{n, q} u_{q} \in d_{n} \cdot R_{\langle K, v>}[X]$

e portanto $\quad v\left(a_{n+1, i}-a_{n, i}\right) \geq v\left(d_{n}\right)=r_{n}$ para $i=0, \ldots, r$

e analogamente $v\left(b_{n+1}, j-b_{n, j}\right) \geq r_{n}$ para $j=0, \ldots, s$.

Disto segue que se $m>n$, então:

$v\left(a_{m, i}-a_{n, i}\right) \geq \min \left\{v\left(a_{m, i}-a_{m-1}, i\right), \ldots, v\left(a_{n+1}, i^{-a_{n}, i}\right)\right\} \geq \gamma_{n} \rightarrow \infty$

e portanto $\left\{a_{n, i}\right\}_{n \in \mathbb{N}} \quad \vec{e}$ de Cauchy para $i=0, \ldots, r$

analogamente $\quad\left\{b_{n, j}\right\}_{n \in \mathbb{N}}$ é de Cauchy para $j=0, \ldots, s$

Como $\langle K, v, \mathbb{Z}\rangle$ e completo, existem:

$a_{i}^{*}=\lim _{n \rightarrow \infty} a_{n, i}$ para $i=0, \ldots, r$ e $b_{j}^{*}=\lim _{n \rightarrow \infty} b_{n, j} \quad \operatorname{para} j=0, \ldots, s$

Sejam $g^{*}=\sum_{i=0}^{r} a_{i}^{*} X^{i} \quad$ e $h^{*}=\sum_{j=0}^{S} b_{j}^{*} X^{j}, \quad$ então $\overline{g^{*}}=\overline{g_{2}}$

$\overline{h^{*}}=\bar{h}$ e fazendo $\sum_{\mathrm{q}=0}^{\mathrm{r}+\mathrm{s}} \mathrm{p}_{\mathrm{q}}^{*} \mathrm{X}^{\mathrm{q}}=\mathrm{f}-\mathrm{g}^{*} \cdot \mathrm{h}^{*} \quad$ tem-se que:

dado $M>0$, existe $n \in \mathbb{N}$ tal que $\gamma_{n}>M, \quad v\left(a_{i}^{*}-a_{n, i}\right)>M$

para $i=0, \ldots, r$ e $v\left(b_{j}^{*}-b_{n, j}\right)>M$ para $j=0, \ldots, s$.

E portanto de:

$\sum_{q=0}^{r+s} p_{q}^{*} x^{q}=f-g^{*} h^{*}=\left(f-g_{n} h_{n}\right)+\left(g_{n}-g^{*}\right) h_{n}+g^{*}\left(h_{n}-h^{*}\right)$,

segue que $v\left(p_{q}^{*}\right)>M$ para $q=0, \ldots, r+s$.

Como isto vale para todo $M>0$ segue que $v\left(\mathrm{p}_{\mathrm{q}}^{*}\right)=\infty$ para $\mathrm{q}=0, \ldots, \mathrm{r}+\mathrm{s}$ ou $\mathrm{p}_{\mathrm{q}}^{*}=0$ para $\mathrm{q}=0, \ldots, \mathrm{r+s}$ e portanto $\mathrm{f}=\mathrm{g}^{*} \cdot \mathrm{h}^{*}$, donde segue em particular que $\operatorname{gr}\left(\mathrm{g}^{*}\right)=\operatorname{gr}(\mathrm{g})=\mathrm{r}$; terminando 
assim a demonstração de que $\langle K, v, \mathbb{Z}\rangle$ satisfaz o Lema de Hense1.

o completado de $\left\langle Q, v_{p}, \mathbb{Z}\right\rangle \vec{e}$ denotado por $\left\langle Q_{p}, v_{p}, \mathbb{Z}\right\rangle$ (observe que usa-se a mesma letra ${ }_{p}$ para a valoração de $\mathrm{Q}_{\mathrm{p}}$ ). Pelo teorema $\mathrm{I} 4.1<\mathrm{Q}_{\mathrm{p}}, \mathrm{v}_{\mathrm{p}}, \mathbb{Z}>$ satisfaz o Lema de Hensel e devido à proposição I4.1, $Q_{p}$ pode ser escrito como: $\mathrm{Q}_{\mathrm{p}}=\left\{\sum_{i=\ell}^{\infty} \mathrm{c}_{i} \mathrm{p}^{\mathrm{i}}: \ell \in \mathbb{Z}, \mathrm{c}_{i} \in\{0,1, \ldots, \mathrm{p}-1\}\right\}, \mathrm{Q}_{\mathrm{p}} \quad \overline{\mathrm{e}}$ conhecido como o corpo dos nümeros p-ädicos.

O completado de $\left\langle K(X), v_{X}, \mathbb{Z}\right\rangle$ do exemplo 2. da $1^{\text {a }}$ seção, com $p=X \in K[X]$ será pela proposição $[4.1$, usando $L=K$ e $p=X$ : $K(X)=\left\{\sum_{i=\ell}^{\infty} c_{i} X^{i}: \ell \in \mathbb{Z}, c_{i} \in K\right\}$ que è precisamente o corpo $K((X))$ das séries formais com coeficientes em $K$. A valoração $\hat{v}_{X}$ de $K(X)$ serâ também a valoração ord de $K((X))$ definida no exemplo 4 . da $1^{a}$ seção. De modo que o completado de $\left\langle K(X), v_{X}, \mathbb{Z}\right\rangle \quad \vec{e}<K((X))$, ord, $\left.\mathbb{Z}\right\rangle$.

Em geral dado um corpo valorado $\langle K, v, \mathbb{Z}\rangle$, se existir algum subcorpo $L$ de $K$ contido em $\left.R_{\langle K, v}\right\rangle$ e tal que $L \simeq \bar{L}=\bar{K}_{V}$, então pela proposição I4.1, $\mathbb{R}=L((p))$ e portanto $\langle\hat{R}, \hat{v}, \mathbb{Z}\rangle$ $\vec{e}$ analiticamente isomorfo a $\left\langle\widetilde{K}_{V}((X))\right.$,ord, $\left.\mathbb{Z}\right\rangle$ (observe que pelo corolärio 4 da prop.I3.1, p ë transcendente sobre L, pois $V(p)=1 \neq 0$; de modo que $L((p)) \simeq \vec{K}_{V}((X))$ como corpos) 
No capítulo II veremos que esta situação de fato ocorre se $\operatorname{car} \bar{K}_{V}=0$ e $\langle K, v, \mathbb{Z}\rangle$ satisfaz o Lema de Hensel. Logo pode mos dizer (devido tambëm ao teorema I4.1) que dado um corpo $\bar{K}$ de caracteristica zero, existe um ünico (a menos de isomor fismos analiticos) corpo valorado completo $\langle\mathrm{K}, \mathrm{v}, \mathbb{Z}\rangle$ com $\overline{\mathrm{K}}_{\mathrm{V}} \cong \overline{\mathrm{K}}$ que é precisamente o corpo $\langle\bar{K}((X))$, ord, $\mathbb{Z}\rangle$.

Para o caso em que car $\overline{\mathrm{K}}=\mathrm{p} \neq 0$ valem os seguintes resulta dos:

Existe um ünico ( a menos de isomorfismos analíticos) cor po valorado completo $\langle K, v, \mathbb{Z}\rangle$ tal que carK $=p$ e $\bar{K}_{v} \simeq \bar{K}$, que é precisamente $\langle\bar{K}((X))$, ord, $\mathbb{Z}\rangle \quad$ (veja [Sch], capVII, teor.12)

Existe um ünico (a menos de isomorfismos analiticos) corpo valorado completo $\langle K, v, \mathbb{Z}\rangle$ tal que $\operatorname{car} K=0, v(p)=1$ e $\overline{\mathrm{K}}_{V} \simeq \overline{\mathrm{K}} \quad(\operatorname{veja}[\mathrm{Sch}]$, cap VII, Lema 32 e teor.10 e corol.1) Se $\overline{\mathrm{K}}$ é perfeito, então o corpo $K$ serä (isomorfo a) o corpo dos vetores de Witt de $\overline{\mathrm{K}}$ : $W(\overline{\mathrm{K}})$ (para a construção deste corpo veja o capítulo 6 de [Grb] ).

\section{\$5. CORPOS VALORADOS PSEUDOCOMPLETOS}

Se $\langle F, W, G\rangle$ è uma extensão imediata de $\langle K, v, G\rangle$ e $a \in F$, então podemos encontrar alguma sequência $\left\{a_{n}\right\}_{n \in \mathbb{N}}$ de $K$ tal que $w\left(a_{n}-a\right) \quad \vec{e}$ estritamente crescente em G (veja Prop.I5.1). 
Se G é $Z$, então $w\left(a_{n}-a\right) \rightarrow \infty$ e isto, de acordo com o que vimos na seção anterior, equivale a $a_{n} \rightarrow a$. Disto segue tamberm, a afirmação que fazíamos naquela seção, que se $<\mathrm{K}, \mathrm{v}, \mathbb{Z}\rangle \overrightarrow{\mathrm{e}}$ completo, então ele è maximalmente completo (pois sendo $\left\langle K, v, \mathbb{Z}>\right.$ completo de $a_{n} \rightarrow a$ segue que $a \in K$ e portanto que $F=K$ ).

No caso em que G ë arquimediano, porêm não discreto, de $w\left(a_{n}-a\right)$ estritamente crescente, não se pode mais concluir que $a_{n}+a ;$ e Ostrowski encontrou exemplos de corpos completos que não exam maximalmente completos (veja os comentärios iniciais da $\$ 4$. de [Ko] ).

Mas foi justamente esta propriedade ( $\left.w_{(} a_{n}-a\right)$ estrita mente crescente) que permitiu-1he introduzir, para corpos com grupos de valores quaisquer, os conceitos de pseucolimite de uma sequência e de sequência pseudoconvergente (em analogia a sequência de Cauchy); de modo a obter a equivalência entre os conceitos de corpo pseudocompleto (o anảlogo a corpo com pleto) e corpo maximalmentemcompleto, como veremos nesta se ção.

DEFINICÃO I5.1 - Seja $\lambda$ um ordinal limite e $\left\{a_{\rho}\right\}_{\rho<\lambda} \quad$ uma sequência de elementos de um corpo valorado $\langle K, v, G\rangle$ :

(i) dizemos que um elemento $a \in K$ e um pseudolimite da se quência $\left\{a_{\rho}\right\}_{\rho}<\lambda$ se, para algum ordinal $\lambda_{0}<\lambda$, 
$v\left(a-a_{\rho}\right)<v\left(a-a_{\sigma}\right)$ sempre que $\lambda_{0}<\rho<\sigma<\lambda$.

Quando isto ocorrer diremos também que $v\left(a-a_{p}\right) \vec{e}$ uma sequência eventualmente crescente.

(ii) dizemos que a sequência $\left\{a_{\rho}\right\}_{\rho<\lambda}$ è pseudoconvergente se, para algum ordinal $\lambda_{0}<\lambda, \quad v\left(a_{\sigma}-a_{\rho}\right)<v\left(a_{\tau}-a_{\sigma}\right)$ sempre que $\lambda_{0}<\rho<\sigma<\tau<\lambda$.

Neste caso também, seguindo a convenção anterior, di remos que $v\left(a_{\sigma}-a_{\rho}\right)$ é eventualmente crescente.

DEFINIÇÃO I 5.2

(i) Um corpo valorado $\langle\mathrm{K}, \mathrm{v}, \mathrm{G}\rangle$ se diz $\lambda$-pseudocompleto, onde $\lambda$ é um ordinal limite, quando toda $\lambda$-sequência $\left\{a_{\rho}\right\}_{\rho<\lambda}$ pseuconvergente de $K$ admite pseudolimite em $K$.

(ii) Um corpo valorado $\langle\mathrm{K}, \mathrm{V}, \mathrm{G}\rangle$ se diz pseudocompleto se ele é $\lambda$-pseudocompleto para todo ordinal limite $\lambda$.

Vamos começar estabelecendo, através de alguns lemas, a1guns fatos elementares -porém básicos- sobre esta nova espê cie de convergência.

LEMA I5.1 - Seja $\langle K, v, G\rangle$ um corpo valorado e $\left\{a_{\rho}\right\}_{\rho<\lambda}$ uma sequência de $K$.

(i) Se $\left\{a_{\rho}\right\}_{\rho<\lambda}$ é pseudoconvergente, então $v\left(a_{\rho+1}-a_{\rho}\right)=v\left(a_{\sigma}-a_{\rho}\right)$ eventualmente para $\rho<\sigma \quad$ (isto $\vec{e}$ : existe algum ordinal $\lambda_{0}<\lambda$ tal que $v\left(a_{\rho+1}-a_{\rho}\right)=v\left(a_{\sigma}-a_{\rho}\right)$ sempre que 
$\left.\lambda_{0}<\rho<\sigma<\lambda\right)$.

Denotaremos $v\left(a_{\rho+1}-a_{\rho}\right)$ por $\gamma_{\rho}$.

(ii) Se $\left\{a_{\rho}\right\}_{\rho<\lambda} \quad \vec{e}$ pseudoconvergente e $a \in K$ é tal que $v\left(a-a_{\rho}\right)=\gamma_{\rho}$ event. , então a $\vec{e}$ um pseudolimite de $\left\{a_{\rho}\right\}_{\rho}<\lambda$.

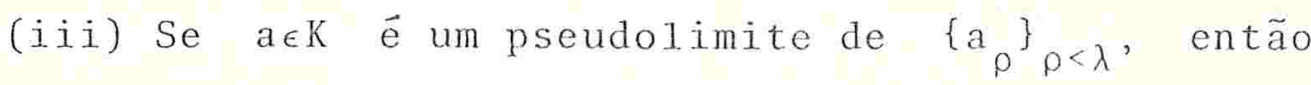
$\left\{a_{\rho}\right\}_{\rho<\lambda} \vec{e}$ pseudoconvergente e $v\left(a-a_{\rho}\right)=\gamma_{\rho}$ event.

\section{DEMONSTRAÇAOO}

(i) Para $\lambda_{0}<\rho<\rho+1<\sigma<\lambda$ (onde $\lambda_{0}$ é o ordinal que aparece na definição de sequência pseudoconvergente) temos:

$v\left(a_{\rho+1}-a_{\rho}\right)<v\left(a_{\sigma}-a_{\rho+1}\right), \quad$ o que permite aplicar-se o lema II.1 (iii) para $v\left(a_{\sigma}-a_{\rho}\right)=v\left(\left(a_{\sigma}-a_{\rho+1}\right)-\left(a_{\rho+1}-a_{\rho}\right)\right)=v\left(a_{\rho+1}-a_{\rho}\right)$

(ii) A sequência $\gamma_{\rho}$ è eventualmente crescente, pois se $\lambda_{0}<\rho<\sigma<\lambda$, então $\gamma_{\rho}=v\left(a_{\rho+1}-a_{\rho}\right)=v\left(a_{\sigma}-a_{\rho}\right)<v\left(a_{\sigma+1}-a_{\sigma}\right)=\gamma_{\sigma}$ eventualmente. Logo se $v\left(a_{-a_{\rho}}\right)=\gamma_{\rho}$ event., então

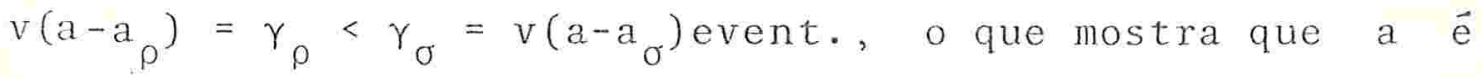
um pseudolimite de $\left\{a_{\rho}\right\} \rho<\lambda$.

(iii) Reciprocamente se a $\vec{e}$ um pseudolimite de $\left\{a_{\rho}\right\}_{\rho<\lambda}$ então para $\lambda_{0}<\rho<\sigma<\lambda$ tem-se $v\left(a_{-}-a_{\rho}\right)<v\left(a-a_{\sigma}\right)$ o que permite aplicar-se o lema I1.1(iii) para obter-se:

$v\left(a_{\sigma}-a_{\rho}\right)=v\left(\left(a_{\sigma}-a\right)+\left(a-a_{\rho}\right)\right)=v\left(a-a_{\rho}\right)$.

Analogamente para $\lambda_{0}<\sigma<\tau<\lambda$ tem-se: $\quad v\left(a_{\tau}-a_{\sigma}\right)=v\left(a_{-}-a_{\sigma}\right)$. Juntando as expressões para $\lambda_{0}<\rho<\sigma<\tau<\lambda$, tem-se:

$v\left(a_{\sigma}-a_{\rho}\right)=v\left(a-a_{\rho}\right)<v\left(a-a_{\sigma}\right)=v\left(a_{\tau} a_{\sigma}\right)$, o que mostra que 
$\left\{a_{\rho}\right\}_{\rho<\lambda} \bar{e}$ pseudoconvergente e que $v\left(a-a_{\rho}\right)=v\left(a_{\sigma}-a_{\rho}\right)=\gamma_{\rho} e v \cdot$

LEMA 15.2 - Seja a um pseudolimite de uma sequência $\left\{a_{\rho}\right\}_{\rho}<\lambda$ Então b è um pseudolimite de $\left\{a_{\rho}\right\}_{\rho<\lambda}$ se e só se $v(a-b) \geq \gamma_{\rho}$ event.

\section{DEMONSTRAÇÃO}

Se a e b saõ pseudolimites de $\left\{a_{\rho}\right\}_{\rho<\lambda}$, então tem-se que: $\quad v\left(a-a_{\rho}\right)=\gamma_{\rho}=v\left(b-a_{\rho}\right)$ event., donde:

$v(a-b) \geq \min \left\{v\left(a-a_{\rho}\right), v\left(a_{\rho}-b\right)\right\} \geq \gamma_{\rho}$ event.

de $v(a-b) \geq \gamma_{\rho}$ event. segue que $v(a-b) \geq \gamma_{\rho+1}>\gamma_{\rho}$ event.

Reciprocamente, se $v(a-b)>\gamma_{\rho}$ event. e a $\vec{e}$ um pseudolimite de $\left\{a_{\rho}\right\}_{\rho}<\lambda$, então $v(a-b)>\gamma_{\rho}=v\left(a-a_{\rho}\right)$ event. e pelo lema I1.1.(iii) tem-se $v\left(b-a_{\rho}\right)=v\left((b-a)+\left(a-a_{\rho}\right)\right)=$ $=v\left(a-a_{\rho}\right)=\gamma_{\rho}$ event., o que mostra que $b$ é um pseudolimite de $\left\{a_{\rho}\right\}<\lambda^{\text {. }}$

LEMA I5.3 - Seja $\left\{a_{\rho}\right\}_{\rho<\lambda}$ uma sequência pseudoconvergente de un corpo valorado $\langle K, v>$.

(i) São equivalentes:

1. zero é um pseudolimite de $\left\{a_{\rho}\right\} \rho<\lambda$

2. $v\left(a_{\rho}\right)=\gamma_{\rho}$ event.

3. $v\left(a_{p}\right) \vec{e}$ eventualmente crescente.

(ii) São equivalentes:

1. zero não é um pseudolimite de $\left\{a_{\rho}\right\} \rho<\lambda$ 


$$
\begin{aligned}
& \text { 2. } v\left(a_{\rho}\right)<\gamma_{\rho} \text { event. } \\
& \text { 3. } v\left(a_{\rho}\right) \vec{e} \text { eventualmente constante. }
\end{aligned}
$$

\section{DEMONSTRAÇAO}

(i) Se $a_{\rho} \rightarrow 0$, então pelo 1ema T5.1(iii), $\quad v\left(a_{\rho}\right)=v\left(0-a_{\rho}\right)=$ $=\gamma_{\rho}$ event., donde segue tambëm que $v\left(a_{\rho}\right)$ e eventualmente crescente. Finalmente se $v\left(a_{\rho}\right)$ e eventualmente crescente, então $v\left(0-a_{\rho}\right)=v\left(a_{\rho}\right)<v\left(a_{\sigma}\right)=v\left(0-a_{\sigma}\right)$ event. e portanto $a_{\rho} \rightarrow 0$. (Observação: faremos uso da notação $a_{\rho}{ }^{+a}$ para pseudolimites sempre que isto não crie confusão como o limite comum descrito na seção anterior. Observe também que não podemos fazer uso da notação a=ps.lim a pois o pseudolimite não é em ge ral único, como se pode ver pelo lema I5.2).

(ii) Seja $\lambda_{0}<\lambda$ o ordinal que aparece na definição de se quência pseudoconvergente. Se a $a_{\rho}+0$, então existem $\lambda_{0}<\rho_{0}<\sigma_{0}$ $<\lambda$ tais que: $v\left(0-a_{\rho_{0}}\right) \geq v\left(0-a_{\sigma_{0}}\right)$. Dai segue que: para $\lambda_{0}<\rho_{0}<\sigma_{0}<\tau<\lambda \quad \gamma_{\sigma_{0}}=v\left(a_{\tau}-a_{\sigma_{0}}\right)>v\left(a_{\sigma}-a_{\rho_{0}}\right) \geq \min \left\{v\left(a_{\sigma_{0}}\right), v\left(a_{\rho_{0}}\right)\right\}$ $=v\left(a_{\sigma_{0}}\right)$ e portanto $\quad v\left(a_{\tau}\right)=v\left(\left(a_{\tau}-a_{\sigma_{0}}\right)+a_{\sigma_{0}}\right)=\min \left\{v\left(a_{\tau}-a_{\sigma_{0}}\right)\right.$, $\left.v\left(a_{\sigma_{0}}\right)\right\}=v\left(a_{\sigma_{0}}\right)$. Com esta argumentação provou-se que: se $a_{\rho} \not 0$, então $v\left(a_{\tau}\right)=v\left(a_{\sigma_{0}}\right)<\gamma_{\sigma_{0}}<\gamma_{\tau}$ isto: è que $1 . \Rightarrow 2 . ;$ e que $2 . \Rightarrow 3$. uma vez que $v\left(a_{\tau}\right)=v\left(a_{\sigma_{0}}\right)$ seguiu apenas de $v\left(a_{\sigma_{0}}\right)<\gamma_{\sigma_{0}}$ que é consequência de $2 \ldots$

Finalmente $3 . \Rightarrow 1$. pois se $v\left(a_{p}\right)$ è eventualmente constante, então pelo item (i) deste lema, zero não pode ser um 
pseudolimite de $\left\{\mathrm{a}_{\rho}\right\} \rho \lambda^{\text {. }}$

Como, dada uma sequência $\left\{a_{\rho}\right\}_{\rho \in \lambda}$, ou $a_{\rho} \rightarrow 0$ ou $a_{\rho} \neq 0$, segue do lema que se $\left\{a_{p}\right\}_{\rho \in \lambda}$ é pseudoconvergente, então ou $v\left(a_{\rho}\right) \quad \vec{e}$ eventualmente crescente ou $v\left(a_{\rho}\right) \quad \vec{e}$ eventualmente constante.

Tambëm juntando os lemas I5.2 e I5.3, segue facilmente que, se $a_{\rho} \rightarrow a$, então:

$$
\begin{array}{lll}
a_{\rho} \rightarrow 0 & \text { se e só se } & v(a)>v\left(a_{\rho}\right)=\gamma_{\rho} \text { event. } \\
a_{\rho} \neq 0 & \text { se e só se } & v(a)=v\left(a_{\rho}\right)<\gamma_{\rho}
\end{array}
$$

Vamos agora demonstrar alguns dos fatos prometidos no início desta seção:

PROPOSIÇÃO I5.1 - Se <F,W,G $\rangle \overrightarrow{\mathrm{e}}$ uma extensão imediata de $\langle K, V, G\rangle$ e $a \in F-K$, então existe alguma sequência pseudoconvergente de $K$, da qual a é um pseudolimite e que não admi te pseudolimites em $K$.

DEMONSTRAÇAOO

Seja $S=\{w(a-z): z \in K\}$. Como $a \not K, \quad a-z \neq 0$ para todo $z \in K \quad$ e portanto $\quad \infty=w(0) \notin S$.

Também S não admite máximo; pois se $\gamma=w(a-z) \epsilon S$, en tão seja $b \in K$ tal que $v(b)=\gamma$ (existe um tal $b \in K$ pois $v(K)=w(F)) . \quad w((a-z) / b)=w(a-z)-w(b)=\gamma-v(b)=0 \quad e$ portanto $(a-z) / b \in R_{<F, w>}$. 
Como $\overline{\mathrm{F}}_{W}=\overline{\mathrm{K}}_{V}$, existe $\mathrm{c} \in \mathrm{R}_{\langle\mathrm{K}, \mathrm{V}\rangle}$ tal que $\mathrm{w}\left(\frac{\mathrm{a}-\mathrm{z}}{\mathrm{b}}-\mathrm{c}\right)>0$ donde $w(a-z-b c)>w(b)=\gamma$ ou $\delta=w\left(a-z^{\prime}\right)>\gamma$, onde $z^{\prime}=z+b c \in K$. E isto mostrou que dado $\gamma \in S$, existe $\delta \in S$, $\delta>\gamma$, confirmando que $S$ não admite máximo.

A Teoria dos Conjuntos garante que dado um conjunto orde nado e não vazio $S$, existe algum subconjunto $S^{\prime}$ de $S$, bem ordenado (com a mesma ordem que $S$ ) e cofinal com $S$ (isto $\vec{e}$ : se $s \in S$, existe $S^{\prime} \in S^{\prime}$ tal que $S^{\prime} S^{\prime}$ ). Para a de monstração deste fato ê suficiente aplicar o Lema de Zorn à familia dos subconjuntos bem ordenados de $\mathrm{S}$.

Sendo S' ben ordenado, poderá ser escrito como: $S^{\prime}=\left\{\gamma_{\rho}: \rho<\lambda\right\}$ onde $\lambda$ será então o ordinal de $S^{\prime}$, e $\left\{\gamma_{\rho}\right\}_{\rho<\lambda}$ será (estritamente) crescente.

No presente caso, como $S$ não admite mäximo, $\lambda$ terá que ser um ordinal limite (senão $\gamma_{\lambda-1}$ seria o mäximo de $S^{\prime}$ e concomitantemente de S).

Sejam $a_{\rho} \in K$ tais que $w\left(a-a_{\rho}\right)=\gamma_{\rho}$. Então a é um pseudolimite de $\left\{a_{\rho}\right\}_{\rho<\lambda}$ uma vez que $\left\{\gamma_{\rho}\right\}_{\rho<\lambda}$ é crescente. Se $b \in K$, então $w(a-b) \in S$ e como $S^{\prime} \vec{e}$ cofinal com $S$, existe $\lambda_{0}<\lambda$ tal que $w(a-b)<\gamma_{\lambda_{0}}<\gamma_{p}$ event. o que mostra, de acordo com o lema I5.2, que b não é pseudolimite de $\left\{a_{\rho}\right\}_{\rho<\lambda}$. 
Esta proposição demonstra a metade da equivalência entre corpos pseudocmpletos e maximalmente completos. Precisamente que todo corpo pseudocompleto è maximalmente completo, pois sendo pseudocompleto não possui sequências pseudoconvergentes sem pseudolimite e portanto, devido à proposição, não pode admitir extensões imediatas pröprias.

Tambêm ve-se pela demonstração a necessidade do uso de sequências de qualquer ordinal, uma vez que o ordinal $\lambda$ é de terminado pelo fato que $\left\{\gamma_{\rho}\right\}_{\rho}<\lambda$ é cofinal com $S$ e este fato é fundamental para a construção de uma sequência sem pseudolimite em $K$, e $\vec{e}$ isto por sua vez que garante o resultado enunciado no parágrafo acima. (é claro que pode-se construir uma sequência enumerâvel com pseudolimite a -basta escolher uma subsequência enumerável de $\left\{a_{\rho}\right\}_{\rho<\lambda^{-}}$mas a $i$ não se poderia garantir que ela não admitirä pseudolimites em K) . COROLARIO - Se <F,w, G> é uma extensão imediata de $\langle\mathrm{K}, \mathrm{v}, \mathrm{G}\rangle$ G é enumerāvel e $a \in F-K$, então existe alguma sequência de K enumerāvel $\left\{\mathrm{a}_{n}\right\}_{n}<\omega$ e pseudoconvergente, da qual a é um pseudolimite e que não admite pseudolimites em K.

(observação: estamos indicando por $\omega$ o ordinal dos naturais) DEMONSTRAÇAO

Como G è enumerảvel, S serả enumerảvel e $S^{\prime}$ tambëm; de modo que o ordinal $\lambda$ de $S^{\prime}$ será precisamente $\omega$ e portanto a sequência $\left\{a_{\rho}\right\}_{\rho<\lambda}$, construida para a demonstração da proposição è enumerável. 
Para a outra metade da equivalência precisamos mostrar como, dada uma sequência pseudoconvergente $\left\{a_{\rho}\right\}_{\rho<\lambda}$ sem pseu dolimite num corpo, construir uma extensão imediata deste cor po, onde a sequência tenha pseudolimites (observe que isto é suficiente para provar que todo corpo maximalmente completo é pseudocompleto).

Para isto, como se verä adiante, ê importante estudar-se o comportamento de $\left\{f\left(a_{\rho}\right)\right\}_{\rho<\lambda}$ quando $f$ é um polinomio com coeficientes no corpo dado.

Recordemos que se $f \in K[X]$ è um polinimio de grau n, então existem $f_{0}, f_{1}, \ldots, f_{n} \in K[X]$ tais que :

$$
\begin{aligned}
f(X+Y)=\sum_{i=0}^{n} f_{i}(X) Y^{i} & \quad \text { (isto se ve desenvolvendo } f(X+Y)= \\
= & \sum_{i=0}^{n} c_{i}(X+Y)^{i} \text { e agrupando por potencias } \\
& \text { de } \left.Y: \sum_{i=0}^{n} c_{i}(X+Y)^{i}=\sum_{i=0}^{n} f_{i}(X) Y^{i}\right) .
\end{aligned}
$$

Derivando sucessivamente em relação a $Y$ e "igualando" $Y$ a zero, obtemos $i: f^{i}(X)=f^{(i)}(X)$. No caso em que cark=0 podemos dividir por i! para obter a familiar fórmula de Taylor: $\quad f(X+Y)=\sum_{i=0}^{n} \frac{f^{(i)}(X)}{i !} Y^{i} ;$ mas em qualquer caso podemos obter as relações: $\quad f_{0}(X)=f(X)$ e $f_{1}(X)=f^{\prime}(X)$ que serão as üicas de que precisaremos. 


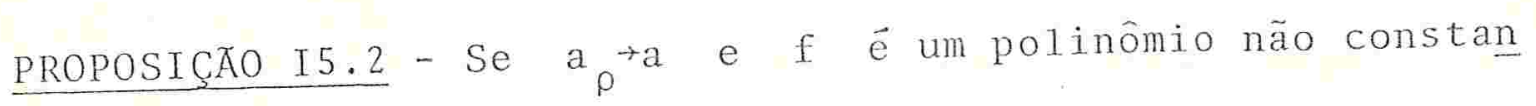
te, então $f\left(a_{\rho}\right)+f(a)$.

\section{DEMONSTRAÇÃO}

Devido ao desenvolvimento de $f$ descrito hä pouco, po de-se escrever:

$$
f\left(a_{\rho}\right)-f(a)=\sum_{i=1}^{n} f_{i}(a) \cdot\left(a_{p}-a\right)^{i}
$$

(com $X+Y=a$ e $X=a)$.

como $f$ não é constante, $f_{i}(a) \neq 0$ para algum $i$ e portanto $v\left(f_{i}(a)\right) \neq \infty$ para algum $i$.

Sejam $\delta_{i}=v\left(f_{i}(a)\right)$ e $\gamma_{\rho}=v\left(a_{\rho}-a\right) \cdot \gamma_{\rho}$ è uma sequência eventualmente crescente, por isso fixados $i \neq j$ (com $f_{i}(a) \neq 0$ ou $\mathrm{f}_{\mathrm{j}}(\mathrm{a}) \neq 0$; e isso sempre é possível) ter-se-a que:

ou $(j-i) \gamma_{\rho}<\delta_{i}-\delta_{j}$ event. ou $(j-i) \gamma_{\rho}>\delta_{i}-\delta_{j}$ event. (uma vez que $j-i$ e $\delta_{i}-\delta_{j}$ estão fixados e sô $\rho$ varia).

Disto segue que: ou $\delta_{j}+j \cdot \gamma_{\rho}<\delta_{i}+i \cdot \gamma_{\rho}$ event. ou $\delta_{j}+j \cdot \gamma_{\rho}>\delta_{i}+i \cdot \gamma_{\rho}$ event. e portanto deve existir algum in dice $1 \leq k \leq n$ tal que $\delta_{k} \neq \infty$ e $\delta_{k}+k \cdot \gamma_{\rho}<\delta_{i}+i \cdot \gamma_{\rho}$ event. pa

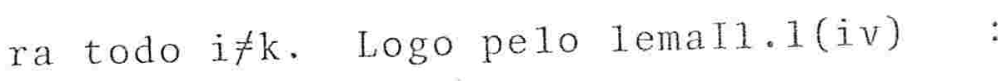

$$
v\left(f\left(a_{\rho}\right)-f(a)\right)=\delta_{k}+k \cdot \gamma_{\rho}=v\left(f_{k}(a) \cdot\left(a_{\rho}-a\right)^{k}\right) \text { event. e por }
$$
tanto $v\left(f\left(a_{\rho}\right)-f(a)\right)=\delta_{k}+k \cdot \gamma_{\rho}<\delta_{k}+k \cdot \gamma_{\sigma}=v\left(f\left(a_{\sigma}\right)-f(a)\right)$ event. se $\rho<\sigma$. (observe que $k \neq 0$, pois $1 \leq k \leq n$, e daí segue a desigualdade $\delta_{k}+k \cdot \gamma_{\rho}<\delta_{k}+k^{\sigma} \gamma_{\sigma}$, uma vez que a sequência $\gamma_{\rho} \vec{e}$ eventualmente crescente). 
A desigualdade $v\left(f\left(a_{\rho}\right)-f(a)\right)<v\left(f\left(a_{\sigma}\right)-f(a)\right)$ event, para $\rho<\sigma \quad \vec{e}$ precisamente a definição de $f\left(a_{\rho}\right) \rightarrow f(a)$.

PROPOSIÇAO I5.3 - Se $\left\{a_{\rho}\right\}_{\rho<\lambda}$ e uma sequência pseudoconvergente de $K$ e $f \in K[X]$ è um polinômio não constante, então $\left\{f\left(a_{\rho}\right)\right\}_{\rho<\lambda} \quad \vec{e}$ uma sequência pseudoconvergente.

DEMONSTRAÇAO

Seja $F$ uma extensão de $K$ contendo todas as raizes dos $f_{i}$ que comparecem no desenvolvimento de $f$, e $\langle F, w\rangle$ uma extensão de $\langle K, v\rangle$ que, conforme citado no início da seção 3 , sempre existe.

Se $a_{\rho} \rightarrow a$ para algum $a \in F$, então, pela proposição anterior, $f\left(a_{\rho}\right) \rightarrow f(a)$ e portanto $\left\{f\left(a_{\rho}\right)\right\}_{\rho<\lambda} \quad \bar{e}$ pseudoconvergente. Se não, então $a_{\rho} \neq a$ e $a_{\rho}-a \neq 0$ para todo $a \in F$ e portan to, pelo lema I5.3(ii), w(a $\left.\rho^{-a}\right)$ é eventualmente constante. Sejam $f_{i}=b_{i} \cdot \prod_{j=1}^{n_{i}}\left(x-r_{j}^{i}\right) \quad$ para $i=1, \ldots, n, \quad x_{j}^{i} \in F$.

Como hâ um numero finito de $x_{j}^{i}$, pode - se escolher o mesmo $\lambda_{0}<\lambda$ tal que $w\left(a_{\rho}-r_{j}^{i}\right)=w\left(a_{\sigma}-r_{j}^{i}\right)$ para $\lambda_{0}<\rho<\sigma<\lambda$ e para todos i, j. $\operatorname{Logo} v\left(f_{i}\left(a_{\rho}\right)\right)=w\left(b_{i}\right)+\sum_{j=1}^{n} w\left(a_{\rho}-r_{j}^{i}\right)=w\left(b_{i}\right)+\sum_{j=1}^{n_{i}} w\left(a_{\sigma}-r_{j}^{i}\right)$ $=v\left(f_{i}\left(a_{\sigma}\right)\right)$ para $\lambda_{0}<\rho<\sigma<\lambda$. Sejam portanto $\delta_{i}=v\left(f_{i}\left(a_{\rho}\right)\right)$ onde $\lambda<\rho$. Novamente algum $\delta_{i} \neq \infty$ pois $f$ não $\vec{e}$ constante. 
De $f\left(a_{\sigma}\right)-f\left(a_{\rho}\right)=\sum_{i=1}^{n} f_{i}\left(a_{\rho}\right) \cdot\left(a_{\sigma}-a_{\rho}\right)^{i}$ e de $v\left(f_{i}\left(a_{\rho}\right) \cdot\left(a_{\sigma}-a_{\rho}\right)^{i}\right)=\delta_{i}+i \cdot \gamma_{\rho}$ para $\lambda_{0}<\rho<\sigma<\lambda$, segue com a mesma argumentação usada na demonstração da proposição anterior que existe $1 \leq k \leq n$ tal que $\delta_{k}+k \cdot \gamma_{\rho}<\delta_{i}+i \cdot \gamma_{\rho}$ event. para $i \neq k$; e portanto $v\left(f\left(a_{\sigma}\right)-f\left(a_{\rho}\right)\right)=\delta_{k}+k \cdot \gamma_{\rho}$ event. para $\rho<\sigma$, donde $v\left(f\left(a_{\sigma}\right) \cdot f\left(a_{\rho}\right)\right)=\delta_{k}+k \cdot \gamma_{\rho}<\delta_{k}+k \cdot \gamma_{\sigma}=$ $=v\left(f\left(a_{\tau}\right)-f\left(a_{\sigma}\right)\right)$ event. se $\rho<\sigma<\tau$ o que mostra que $\left\{f\left(a_{\rho}\right)\right\}_{\rho<\lambda} \quad \bar{e}$ pseudoconvergente.

DEFINICÃO I5.3 - Uma sequência $\left\{a_{\rho}\right\}_{\rho<\lambda}$ pseudoconvergente em K se diz:

(i) Algēbrica em $K$ se existe algum $f \in K[X]$ tal que $f\left(a_{\rho}\right) \rightarrow 0$ (ii) Transcendente em $K$ se $f\left(a_{\rho}\right) \neq 0$ para todo $f \in K[X]$.

Juntando os resultados do lema 15.3 e da proposição 15.3 temos que: $\left\{\mathrm{a}_{\rho}\right\}_{\rho<\lambda} \overrightarrow{\mathrm{e}}$ algëbrica em $K$ se e só se existe algum $f \in K[x]$ tal que $v\left(f\left(a_{\rho}\right)\right)$ é eventualmente crescente. $E \quad\left\{a_{\rho}\right\}_{\rho<\lambda}$ é transcendente em $K$ se e só se $v\left(f\left(a_{\rho}\right)\right) \vec{e}$ eventualmente constante para todo $f \in K[X]$.

Quando $v\left(f\left(a_{p}\right)\right)$ for eventualmente constante indicaremos por $\lambda_{f}$ um ordinal tal que $v\left(f\left(a_{\rho}\right)\right)=v\left(f\left(a_{\sigma}\right)\right)$ sempre que $\lambda_{\mathrm{f}}<\rho<\sigma<\lambda$. 
TEOREMA I5.1 - Seja $\left\{\mathrm{a}_{\rho}\right\}_{\rho<\lambda}$ uma sequência pseudoconvergente transcendente em K (e portanto não admite pseudolimite em K). Então:

(i) $\left\langle K(X), w>\right.$, onde $w \vec{e}$ definida a partir de $w(f)=v\left(f\left(a_{p}\right)\right)$ para $\lambda_{f}<p<\lambda$ e $w(f / g)=w(f)-W(g), \quad \vec{e}$ uma extensão imediata de $\langle K, v\rangle$ e $a_{\rho} \rightarrow X$ em $\langle K(X), W\rangle$.

(ii) Se $\langle K(a), u\rangle$ è uma extensão de $\langle K, v\rangle$ e $a_{p} \rightarrow a$ em $<K(a), u\rangle$, então a $\vec{e}$ transcendente sobre $K$ e a aplica ção $\phi: K(X) \rightarrow K(a)$ definida por $\phi(f / g)=f(a) / g(a)$ é um isomorfismo analitico entre os corpos valorados $\langle K(X), w\rangle$ e $\langle K(a), u\rangle$.

\section{DEMONSTRACATO}

(i) Seja $\rho>\lambda_{f}, \lambda_{g}, \lambda_{f} \cdot g$, então $w(f \cdot g)=v\left((f \circ g)\left(a_{\rho}\right)\right)=$ $=v\left(f\left(a_{\rho}\right) \cdot g\left(a_{\rho}\right)\right)=v\left(f\left(a_{\rho}\right)\right)+v\left(g\left(a_{\rho}\right)\right)=w(f)+w(g)$.

Seja $\rho>\lambda_{f}, \lambda_{g}, \lambda_{f+g}$, então $w(f+g)=v\left(f\left(a_{\rho}\right)+g\left(a_{\rho}\right)\right) \leq$ $\leq \min \left\{v\left(f\left(a_{\rho}\right)\right), v\left(g\left(a_{\rho}\right)\right)\right\}=\min \{w(f), w(g)\}$. Logo $w$ satisfaz as condições de uma valoração em $K[x]$ e a partir disso è fäcil ver que é uma valoração de $K(X)$.

Se $f=c \neq 0$ è um polinomio constante, então $w(c)=w(f)=$ $=v\left(f\left(a_{\rho}\right)\right)=v(c)$ e portanto $w$ extende $v$.

o grupo de valores $w(K(X))$ é o mesmo que $v(K)$ como se vê imediatamente da definição de w. Falta então verificar que $\overline{K(X)}{ }_{W}=\bar{K}_{V}$ para se concluir que $\langle K(X), W\rangle$ é una exten são imediata de $\langle K, v\rangle$. 
Se $f \in K[X]$ è um polinômio não constante, então pelo lema I5.3(ii), aplicado à sequência $\left\{f\left(a_{\rho}\right)\right\}_{p<\lambda}$, tem-se que, para algum $\lambda_{0}<\lambda, \quad v\left(f\left(a_{\rho}\right)\right)<\gamma_{\rho}=v\left(f\left(a_{\sigma}\right)-f\left(a_{\rho}\right)\right)$ sempre que $\lambda_{0}<\rho<\sigma<\lambda$.

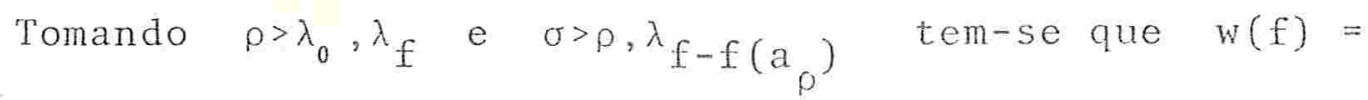
$=v\left(f-f\left(a_{\rho}\right)\right)$ e $w\left(f-f\left(a_{\rho}\right)\right)=v\left(f\left(a_{\sigma}\right)-f\left(a_{\rho}\right)\right)$ e portanto $w(f)<w\left(f-f\left(a_{\rho}\right)\right)$.

Se $f=c \neq 0$ è um polinomio constante, então tambẻm $W(f)=V(c)<\infty=V(C-c)=W(f-c)$.

De qualquer modo dado $0 \neq f \in K[X]$, existe $c_{f} \in K$ tal que $w(f)<w\left(f-c_{f}\right)$. Disto, e com um pouco de manipulação algébri ca, demonstra-se que: $w(f / g)<w\left(f / g-c_{f} / c_{g}\right)$.

Logo dado $f / g \in R_{\langle K}(X), W>$, existe $c=c_{f} / c_{g} \in K$ tal que $w(f / g-c)>W(f / g) \geq 0, \quad$ o que mostra que $\overline{K(X)_{W}}=\bar{K}_{V}$.

Finalmente $a_{\rho} \rightarrow X$, pois tomando $f=X-a_{\rho}$ para $\lambda_{0}<\rho<\lambda$ ( $\lambda_{0}$ o ordinal da definição de sequência pseudoconvergente) tem-se para $\sigma>\rho, \lambda_{f}$ que:

$w\left(X-a_{\rho}\right)=w(f)=v\left(f\left(a_{\sigma}\right)\right)=v\left(a_{\sigma}-a_{\rho}\right)=\gamma_{\rho} \cdot$

(ii) Se a fosse algébrico sobre $K$, então para algum $0 \neq \mathrm{g} \in \mathrm{K}[\mathrm{X}], \quad \mathrm{g}(a)=0$. De $\mathrm{a}_{\rho} \rightarrow a$ seguiria, da proposição I5.2, que $g\left(a_{\rho}\right) \rightarrow g(a)=0 ; \quad$ o que contradiz a definição de sequência transcendente.

Sendo a transcendente sobre $K$ a aplicação $\phi: K(X) \rightarrow K(a)$ 
$\vec{e}$ um isomorfismo (algébrico) entre os dois corpos. Para que $\phi$ seja um isomorfismo analítico e suficiente então que mante nha as valorações, isto é: $w(f / g)=u(\phi(f / g))$. E claro tam bëm que ë suficiente mostrar que $w(f)=u(\phi(f))=u(f(a))$ pa ra $f \in K[X]$.

De $a_{\rho} \rightarrow a$ segue que $f\left(a_{\rho}\right) \rightarrow f(a)$ e como $f\left(a_{\rho}\right) \leftrightarrow 0$, então pelo comentário apös o lema I5.3, segue que:

$u(f(a))=u\left(f\left(a_{\rho}\right)\right)$ event. e portanto:

$u(f(a))=u\left(f\left(a_{\rho}\right)\right)=v\left(f\left(a_{\rho}\right)\right)=w(f)$. Cobserve que em particu lar $\langle K(a), u\rangle$ è uma extensão imediata de $\langle K, v\rangle$ ).

TEOREMA $15.2-$ Seja $\left\{a_{\rho}\right\}_{\rho<\lambda}$ uma sequência pseudoconvergente algëbrica em K.

(i) Seja $q \in K[X]$ um polinômio de grau mínimo tal que $q\left(a_{\rho}\right) \rightarrow 0$. Então q é irredutível em $K[X]$ e $\langle K[X] / q \cdot K[X], w\rangle$, onde $w \vec{e}$ definida por $w(f+q K[X])=v\left(f\left(a_{\rho}\right)\right)$ para $\lambda_{f}<\rho \quad e$ $\operatorname{gr}(f)<\operatorname{gr}(q)$, ê uma extensão imediata de $\langle K, v\rangle$ tal que $\mathrm{a}_{\rho} \rightarrow \mathrm{X}+\mathrm{q} \cdot \mathrm{K}[\mathrm{X}]$ em $\langle K[X] / \mathrm{q} \cdot \mathrm{K}[\mathrm{X}], \mathrm{w}\rangle$.

(o polinômio q nestas condições se diz um polinômio minimal de $\left.\left\{a_{\rho}\right\}_{\rho<\lambda}\right)$.

(ii) Se $\langle K(a), u\rangle$ é uma extensão de $\langle K, v\rangle$ e $a_{p} \rightarrow a$ em $<K(a), u>$ e $q(a)=0$, então $\phi: K[X] / q \cdot K[X]+K(a)$ definida por $\phi(f+q \cdot K[X])=f(a)$ e um isomorfismo analitico. 


\section{DEMONSTRAÇAOO}

(i) Se q=f.g em $K[x]$ com $g r(f), g r(g)<g r(q)$, en tão $f\left(a_{\rho}\right) \nRightarrow 0$ e $g\left(a_{\rho}\right) \nRightarrow 0$, donde $v\left(f\left(a_{\rho}\right)\right)$ e $v\left(g\left(a_{\rho}\right)\right)$ são eventualmente constantes e portanto $v\left(q\left(a_{p}\right)\right)=v\left(f\left(a_{p}\right)\right)+$ + $v\left(g\left(a_{\rho}\right)\right)$ também seria eventualmente constante, o que pe10 lema 15.3 contradiz $q\left(a_{\rho}\right) \rightarrow 0$. Logo q é irredutível em $K[X]$.

$K[X] / q \cdot K[X]$ è o quociente do anel K[X] pelo seu ideal maximal gerado por $q: q \cdot K[X]$. E portanto um corpo e sabese que os elementos de $K[X] / q \cdot K[X]$ podem ser escritos de modo único como $f+q \cdot K[X]$, onde $f=0$ ou $\operatorname{gr}(f)<\operatorname{gr}(q)$. Tambëm se $g r(f)<g r(q)$, então $f\left(a_{\rho}\right)$ \& 0 e portanto $v\left(f\left(a_{\rho}\right)\right)$ é eventualmente constante, o que mostra que w es tá bem definida.

Sejam $\left(f_{1}+q \cdot K[X]\right) \cdot\left(f_{2}+q \cdot K[X]\right)=f+q \cdot K[X]$ com $\operatorname{gr}\left(f_{1}\right), \operatorname{gr}\left(f_{2}\right), \operatorname{gr}(f)<\operatorname{gr}(q)$. Então $f_{1} \cdot f_{2}-f \in q \cdot K[X]$ e portanto $f_{1} \cdot f_{2}-f=p \cdot q$ para algum $p \in K[X]$.

se para $\rho>\lambda_{f_{1}}, \lambda_{f_{2}}, \lambda_{f} \quad v\left(f_{1}\left(a_{\rho}\right) \cdot f_{2}\left(a_{\rho}\right)\right) \neq v\left(f\left(a_{\rho}\right)\right)$, então pe1o lema I1.1(iii), $\quad v\left(f_{1}\left(a_{p}\right) \cdot f_{2}\left(a_{p}\right)-f\left(a_{p}\right)\right)=$ $=\min \left\{v\left(f_{1}\left(a_{\rho}\right) \cdot f_{2}\left(a_{\rho}\right)\right), v\left(f\left(a_{\rho}\right)\right)\right\}$ e portanto $v\left(f_{1}\left(a_{p}\right) \cdot f_{2}\left(a_{p}\right)-f\left(a_{\rho}\right)\right)$ seria eventualmente constante. Mas $v\left(f_{1}\left(a_{p}\right) \cdot f_{2}\left(a_{p}\right)-f\left(a_{p}\right)\right)=v\left(p\left(a_{p}\right) \cdot q\left(a_{p}\right)\right)=v\left(p\left(a_{p}\right)\right)+$ $+v\left(q\left(a_{\rho}\right)\right)$ que $\vec{e}$ eventualmente crescente, pois $v\left(q\left(a_{\rho}\right)\right) \vec{e}$ eventualmente crescente e $v\left(p\left(a_{\rho}\right)\right)$ é event. crescente ou 
event. constante. Logo deve-se ter $v\left(f_{1}\left(a_{\rho}\right) \cdot f_{2}\left(a_{p}\right)\right)=$ $v\left(f\left(a_{\rho}\right)\right)$ para $p>\lambda_{f_{1}}, \lambda_{f_{2}}, \lambda_{f}$ e portanto:

$w\left(f_{1}+q \cdot K X\right)+w\left(f_{2}+q \cdot K X\right)=v\left(f_{1}\left(a_{\rho}\right)\right)+v\left(f_{2}\left(a_{\rho}\right)\right)=$

$=v\left(f_{1}\left(a_{\rho}\right) \cdot f_{2}\left(a_{\rho}\right)\right)=v\left(f\left(a_{\rho}\right)\right)=w(f+q \cdot K[X])=$

$=w\left(\left(f_{1}+q \cdot K[X]\right) \cdot\left(f_{2}+q \cdot K[X]\right)\right)$.

A outra condição, $\quad w\left(\left(f_{1}+q \cdot K[X]\right)+\left(f_{2}+q \cdot K[X]\right)\right) \geq$ $\geq \min \left\{w\left(f_{1}+q \cdot K[X]\right), w\left(f_{2}+q \cdot K[X]\right), \quad \vec{e}\right.$ imediata a partir de definição de .

Os grupos de valores e os corpos das classes residuais coincidem; e a demonstração è análoga à do teorema anterior, pois aqui tambêm vale que $w(f+q \cdot K[X])<w\left((f+q \cdot K[X])-f\left(a_{\rho}\right)\right)$.

E da mesma forma $a_{\rho} \rightarrow X+q \cdot K[X]$ pois:

$w\left((X+q \cdot k[X])-a_{\rho}\right)=v\left(a_{\sigma}-a_{\rho}\right)=r_{\rho}$ event.

(ii) Como $q(a)=0$ e $q$ è irredutível sobre $K$, a ap 1 i cação $\phi \vec{e}$ um isomorfismo (algêbrico) entre os corpos $K[X] / q \cdot K[X]$ e $K(a)$. E um isomorfismo analitico, pois $u(\phi(f+q \cdot k[X]))=u(f(a))=u\left(f\left(a_{\rho}\right)\right)=v\left(f\left(a_{\rho}\right)\right)=w(f+q K[X])$ uma vez que aqui também $f\left(a_{\rho}\right) \rightarrow f(a)$ e $f\left(a_{\rho}\right) \nrightarrow 0$.

Finalmente, a proposição 15.1 e as partes (i) dos teore-mas 15.1 e 15.2 demonstram o:

TEOREMA I5.3 - Um corpo valorado è maximalmente completo se e sö se è pseudocompleto. 
Kaplansky mostrou que se $\operatorname{car} \bar{K}_{V}=0$ ou se $\operatorname{car} \bar{K}_{V}=p \neq 0$ e es tá satisfeita uma condição adicional que chamou de Hipótese A (que é: $p \cdot G=G$ e todo polinomio de forma $x^{p^{n}}+a x^{p^{n-1}}+\ldots+a_{n-1} x^{p}+a_{n} x+a_{n+1} \in \bar{K}_{v}[x]$ admite raiz em $\overline{\mathrm{K}}_{\mathrm{v}}$ ), então as extensões imediatas maximais de $\left.<\mathrm{K}, \mathrm{v}, \mathrm{G}\right\rangle$ são a naliticamente isomorfas. E apresentou contra-exemplos para o caso em que falhava a Hipötese A (veja [Kp]).

A chave desta demonstração está dada pelas partes (ii) dos teoremas 15.1 e I5.2; e a dificuldade consiste em que quando $\left\{a_{\rho}\right\}_{\rho<\lambda} \vec{e}$ algëbrica, para se garantir o isomorfismo a nalítico entre uma extensão $\langle K(a), u\rangle$ de $\langle K, v\rangle$ onde a é um pseudolimite de $\left\{a_{\rho}\right\}_{\rho<\lambda}$ e a extensão padräo $\langle K[X] / q \cdot K[X], w\rangle$ é necessārio que $\mathrm{q}(a)=0$. Kaplansky usou a Hipötese A para mostrar que dada uma sequência pseudoconvergente algébrica de $\langle K, v\rangle$ e uma extensão $\langle F, W\rangle$ pseudocompleta de $\langle K, v\rangle$, então existe $a \in F$ pseudolimite da sequência e tal que $q(a)=0$ (onde q é um polinômio minimal da sequência).

Ax e Kochen, para o seu teorema de isomorfismo (que apre sentamos no $2^{\circ}$ Capitulo), precisaram demonstrar o mesmo fato, porém a partix de uma outra condição que a princípio chama ram de Hipótese $B$ ([AK, II]), que é precisamente a de corpo finitamente ramificado (Kochen em [Ko] introduz este nome); e é justamente este desenvolvimento que veremos agora, se guindo $\left[K_{\mathrm{O}}\right]$. 
LEMA 15.4 - Sejam $\langle K, v\rangle$ um corpo valorado finitamente rami ficado, $\langle\mathrm{F}, \mathrm{W}\rangle$ uma extensão de $\langle\mathrm{K}, \mathrm{v}\rangle, \quad\left\{\mathrm{a}_{\rho}\right\}_{\rho}<\lambda$ uma sequên cia pseudoconvergente de $K$ e $f \in K[X]$ um polinômio não constan te, com $f^{\prime}$ tambëm não constante e tal que $f^{\prime}\left(a_{\rho}\right)$ \& 0 . Se $a \in F$ e um pseudolimite de $\left\{a_{\rho}\right\}<\lambda$, então: $w\left(f\left(a_{\rho}\right)-f(a)\right)=w\left(f^{\prime}(a)\right)+\gamma_{\rho}$ event. (onde $\gamma_{\rho}=v\left(a_{\rho+1}-a_{\rho}\right)=w\left(a-a_{\rho}\right)$ event. ). DEMONSTRACÃO

Pela demonstração da proposição I5.2, $w\left(f\left(a_{\rho}\right)-f(a)\right)=$ $\delta_{k}+k \cdot \gamma_{\rho}$ event., onde $\delta_{i}=w\left(f_{i}(a)\right)$ para $1 \leq i \leq r=g r f$, $f_{i}(a)$ são os coeficientes do desenvolvimento de $f(a+X)$ (em particular $\left.w\left(f^{\prime}(a)\right)=w\left(f_{1}(a)\right)=\delta_{1}\right)$ e $1 \leq k \leq r$ era tal que $\delta_{k}+k \cdot \gamma_{\rho}<\delta_{i}+i \cdot \gamma_{\rho}$ event. para todo $i \neq k, 1 \leq i \leq r$.

o mesmo raciocínio pode-se aplicar para : $f^{\prime}\left(a_{\rho}\right)-f^{\prime}(a)=\sum_{i=2}^{r} f_{i}(a) \cdot i \cdot\left(a_{\rho}-a\right)^{i-1}$, pois algum dos $f_{i}(a) \cdot i \neq 0$ (senão $f^{\prime}$ seria constantemente $f^{\prime}(a)$ ) ; obtendose $w\left(f^{\prime}\left(a_{\rho}\right)-f^{\prime}(a)\right)=\delta_{j}+v(j)+(j-1) \cdot \gamma_{\rho}$ event., onde $\delta_{j}+v(j)+(j-1) \cdot \gamma_{\rho}<\delta_{i}+v(i)+(i-1) \cdot \gamma_{\rho}$ event. para todo $i \neq j, \quad 2 \leq i \leq r$.

Como $f^{\prime}\left(a_{\rho}\right) \rightarrow f^{\prime}(a) \quad\left(\right.$ prop.I5.2) e $f^{\prime}\left(a_{\rho}\right) \nrightarrow 0$, segue do comentário após o 1ema I5.3, que $w\left(f^{\prime}(a)\right)=w\left(f^{\prime}\left(a_{p}\right)\right) e v$. Logo: $\quad w\left(f^{\prime}(a)\right)=\min \left\{w\left(f^{\prime}(a)\right), w\left(f^{\prime}\left(a_{\rho}\right)\right)\right\} \leq w\left(f^{\prime}\left(a_{\rho}\right)-f^{\prime}(a)\right)=$ $=\delta_{j}+v(j)+(j-1) \gamma_{\rho}$ event. ou $\delta_{1} \leq \delta_{j}+v(j)+(j-1) \gamma_{\rho}$ event. 
e portanto $\delta_{1} \leq \delta_{i}+v(i)+(i-1) \gamma_{\rho}$ event. para todo $2 \leq i \leq r$. E como $\gamma_{\rho}<\gamma_{\rho+1}$ event., segue então que $\delta_{1}<\delta_{i}+v(i)+(i-1) \gamma_{\rho}$ event. para todo $2 \leq i \leq r$.

Se o Indice de ramificação de $\langle K, v\rangle$ e zero, então $\operatorname{car} \bar{K}_{V}=0$ e portanto $v(i)=0$ para todo inteiro $i \neq 0 ;$ donde $\delta_{1}<\delta_{i}+(i-1) \gamma_{\rho}$ event. para todo $2 \leq i \leq r$.

Senão, pela proposição 12.4 , para cada inteiro $i \neq 0 \quad 0$ conjunto: $\{g \in v(K): 0<g \leq v(i)\} \quad \vec{e}$ finito. Seja $n_{i} \geq 0$ o nu mero de elementos daquele conjunto e seja $\lambda_{0}<\lambda$ tal que $\gamma_{\rho}<\gamma_{\rho+1}$ para $\lambda_{0}<\rho<\lambda$, então $\gamma_{\rho}<\gamma_{\rho+1}<\gamma_{\rho+2}<\ldots<\gamma_{\rho+n_{i}}$ para $\lambda_{0}<\rho<\lambda$ e portanto $0<\gamma_{\rho+1}-\gamma_{\rho}<\gamma_{\rho+2}-\gamma_{\rho}<\ldots<$ $<\gamma_{\rho+n_{i}}-\gamma_{\rho}$, isto ê existem $n_{i}$ elementos de $v(K)$ menores ou iguais a $\gamma_{\rho+n_{i}}-\gamma_{\rho}$. Logo $v(i) \leq \gamma_{\rho+n_{i}}-\gamma_{\rho} \quad$ ou $v(i)+\gamma_{\rho} \leq \gamma_{\rho+n_{i}}$

Se $2 \leq i \leq r$, então $i-2 \geq 0$ e portanto: $v(i)+(i-1) \gamma_{\rho}=v(i)+\gamma_{\rho}+(i-2) \gamma_{\rho} \leq \gamma_{\rho}+n_{i}+(i-2) \gamma_{\rho}+n_{i}=$ $=(i-1) \gamma_{\rho+n_{i}} \cdot$ Escolhendo $n=\max \left\{n_{2}, \ldots, n_{r}\right\} \quad$ segue que: $v(i)+(i-1) \gamma_{\rho} \leq(i-1) \gamma_{\rho+n}$ para todo $2 \leq i \leq r$ e $\lambda_{0}<\gamma<\rho$. Seja $\lambda_{1}<\lambda$ tal que $\delta_{1}<\delta_{i}+v(i)+(i-1) \gamma_{\rho}$ para todo $2 \leq i \leq r \quad$ e $\quad \lambda_{1}<\rho<\lambda$. Se $\lambda_{2}=\max \left\{\lambda_{0}, \lambda_{1}\right\}$, então $\delta_{1}<\delta_{i}+v(i)+(i-1) \gamma_{p}<\delta_{i}+(i-1) \gamma_{\rho+n}$ para todo $2 \leq i \leq r \quad$ e 
$\lambda_{2}<\rho<\lambda$ e portanto $\delta_{1}<\delta_{i}+(i-1) \gamma_{p}$ para todo $2 \leq i \leq r \quad$ e $\lambda_{2}+n<p<\lambda$, isto $\vec{e} \quad \delta_{1}<\delta_{i}+(i-1) \gamma_{\rho}$ event.

Logo em qualquer caso $(e=0$ ou $0<\mathrm{e}<\infty) \quad \delta_{1}<\delta_{i}+(i-1) \gamma_{\rho} e v$ donde $\delta_{1}+\gamma_{\rho}<\delta_{i}+i \cdot \gamma_{\rho}$ event. para $2 \leq i \leq r$ e portanto $k=1$ na expressão $w\left(f\left(a_{\rho}\right)-f(a)\right)=\delta_{k}+k \cdot \gamma_{p}$ event., ou seja: $w\left(f\left(a_{\rho}\right)-f(a)\right)=\delta_{1}+1 \cdot \gamma_{\rho}=w\left(f^{\prime}(a)\right)+\gamma_{\rho}$ event.

PROPOSICAOO I5.4 - Sejam $\langle K, v\rangle$ um corpo valorado finitamente ramificado, $\left\{\mathrm{a}_{\rho}\right\}_{\rho<\lambda}$ uma sequência pseudoconvergente a1gébrica em K, com polinômio minimal q $\in K[X]$ e que não admite pseudolimites em $K$. Se $\langle F, w\rangle$ è uma extensão de $\langle K, v\rangle$ que satisfaz o Lema de Hensel e existe a $\in$ F pseudolimite de $\left\{a_{\rho}\right\}_{\rho<\lambda}$, então existe $b \in F$ pseudolimite de $\left\{_{\rho}\right\}_{\rho \leqslant \lambda}$ tal que $q(b)=0$.

\section{DEMONSTRAÇAO}

$\operatorname{gr}(q) \geq 2$, pois se $\operatorname{gr}(q)=1 \quad(g r(q) \neq 0$, pois q não é constante, senão $q\left(a_{\rho}\right) \nRightarrow 0$ e pelo mesmo motivo $\left.q \neq 0\right)$, então $\mathrm{q}=\mathrm{c}_{0}+\mathrm{c}_{1} \mathrm{X}$ com $\mathrm{c}_{1} \not \overrightarrow{0}$ e de $\mathrm{q}\left(\mathrm{a}_{\rho}\right) \rightarrow 0$, seguiria que:

$a_{p} \rightarrow-\left(c_{0} / c_{1}\right) \in K$.

Logo $\operatorname{gr}\left(\mathrm{q}^{\prime}\right) \geq 1$, pois $\operatorname{cark}=0$, uma vez que $\mathrm{K} \overrightarrow{\mathrm{e}}$ finita mente ramificado; e portanto q' não ê constante. Tambëm $q^{\prime}\left(a_{\rho}\right) \nrightarrow 0$, senão q não seria um polinômio de grau mínimo tal que $q\left(a_{\rho}\right) \rightarrow 0$; e portanto $q^{\prime}(a) \neq 0$, pois $q^{\prime}\left(a_{\rho}\right) \rightarrow q^{\prime}(a)$. Se $q(a)=0$, então $b=a$ e a proposição estâ demonstrada. 
Se $q(a) \neq 0$, seja $f=\frac{1}{q(a)} q\left(a+\frac{q(a)}{q^{\prime}(a)} x\right) \in F[x]$.

Pelo desenvolvimento de Taylor:

$$
\begin{aligned}
q\left(a+\frac{q(a)}{q^{\prime}(a)} x\right)=\sum_{i=0}^{r} q_{i}(a) \frac{q(a)^{i}}{q^{\prime}(a)^{i}} x^{i} & =q_{0}(a)+q_{1}(a) \frac{q(a)}{q^{\prime}(a)} x+ \\
& +\sum_{i=2}^{r} q_{i}(a) \frac{q(a)}{q^{\prime}(a)^{i}} x^{i},
\end{aligned}
$$

e como $q_{0}(a)=q(a), \quad q_{1}(a)=q^{\prime}(a)$ :

$q\left(a+\frac{q(a)}{q^{\prime}(a)} x\right)=q(a)+q(a) x+\sum_{i=2}^{r} q_{i}(a) \frac{q(a)^{i}}{q^{\prime}(a)^{i}} x^{i}$

e

portanto $f=1+x+\sum_{i=2}^{r} q_{i}(a) \frac{q(a)^{i-1}}{q^{\prime}(a)^{i}} x^{i}=1+x+\sum_{i=2}^{r} c_{i} x^{i}$

Como q e q' não são constantes e $q^{\prime}\left(a_{p}\right) \nRightarrow 0$, pode-se aplicar o lema anterior para obter-se:

$w\left(q\left(a_{\rho}\right)-q(a)\right)=\delta+\gamma_{\rho}=w\left(q^{\prime}(a)\right)+\gamma_{\rho}$ event. (onde $\left.\gamma_{\rho}=w\left(a-a_{\rho}\right)\right)$

e como $q\left(a_{\rho}\right) \rightarrow q(a)$ e $q\left(a_{\rho}\right) \rightarrow 0$, então pelos comentärios após os lema I5.3, $w(q(a))>w\left(q\left(a_{p}\right)\right)$ event. e portanto, pe10 lema I1.1(ii):

$$
\begin{aligned}
& w(q(a))>w\left(q\left(a_{\rho}\right)\right)=\min \left\{w\left(q\left(a_{\rho}\right)\right), w(q(a))\right\}=w\left(q\left(a_{\rho}\right)-q(a)\right)= \\
& =\delta_{1}+\gamma_{\rho} \text { event., } \quad \text { ou } w(q(a))>\delta_{1}+\gamma_{\rho} \operatorname{event} . \\
& \quad \text { Os coeficientes de } f \text { para } 2 \leq i \leq r \quad \text { são: } c_{i}=q_{i}(a) \frac{q(a)^{i-1}}{q^{\prime}(a)^{i}} \\
& \operatorname{Logo} w\left(c_{i}\right)=w\left(q_{i}(a) \frac{q(a)^{i-1}}{q^{\prime}(a)^{i}}\right)=\delta_{i}+(i-1) w(q(a))-i \cdot \delta_{1}
\end{aligned}
$$

e de $w(q(a))>\delta_{1}+\gamma_{p}$ event. segue: 


$$
\begin{aligned}
w\left(c_{i}\right) & >\delta_{i}+(i-1)\left(\delta_{1}+\gamma_{\rho}\right)^{-}-i \delta_{1}=\delta_{i}+i \gamma_{\rho}-\delta_{1}-\gamma_{\rho}= \\
& =\left(\delta_{i}+i \gamma_{\rho}\right)-\left(\delta_{1}+\gamma_{\rho}\right)>0,
\end{aligned}
$$

o que mostra que $c_{i} \in I_{\langle F, W\rangle}$ e portanto $f \in R_{<F, W\rangle}[X] \quad e$

$$
\begin{aligned}
& \bar{f}=\bar{I}+\mathrm{X} \in \overline{\mathrm{F}}_{\mathrm{W}}[\mathrm{X}] \quad\left(\text { pois } \overline{\mathrm{C}}=\overline{0} \text { em } \overline{\mathrm{F}}_{W} \text { para } 2 \leq \mathrm{i} \leq \mathrm{r}\right. \text { ). } \\
& \overline{\mathrm{I}}=\overline{\mathrm{g}} \cdot \overline{\mathrm{h}} \quad \mathrm{com} \mathrm{g}=1+\mathrm{X}, \mathrm{h}=1 \in \mathrm{R}_{\langle\mathrm{F}, \mathrm{W}\rangle}[\mathrm{X}] \text { e }(\overline{\mathrm{g}}, \overline{\mathrm{h}})=1 .
\end{aligned}
$$

Logo pelo Lema de hensel $f=g^{*} \cdot h^{*} \operatorname{com} \operatorname{gr}\left(g^{*}\right)=1$ e $\overline{\mathrm{g}^{*}}=\bar{I}+X$, isto $\overrightarrow{\mathrm{e}}: \mathrm{g}^{*}=\mathrm{a}_{0}+\mathrm{a}_{1} \mathrm{X} \in \mathrm{R}_{\langle\mathrm{F}, \mathrm{W}\rangle}[X] \mathrm{com}$ $\overline{a_{0}}=\overline{a_{1}}=\bar{I}$ (e portanto $w\left(a_{0}\right)=w\left(a_{1}\right)=0$ ).

Sejam $c=-\left(a_{0} / a_{1}\right) \quad$ e $\quad b=a+\frac{q(a)}{q^{\prime}(a)} c$ Então:

$$
g^{*}(c)=0 \text { e portanto } f(c)=0, \text { donde } q(b)=0 \text {. }
$$$$
w(b-a)=w\left(\frac{q(a)}{q^{\prime}(a)} c\right)=w(q(a))-\delta_{1}+w(c)>\gamma_{\rho} \text { event. }
$$

(pois $\left.w(c)=w\left(a_{0}\right)-w\left(a_{1}\right)=0-0=0\right)$

e portanto, pelo lema I5.2, b ë pseudolimite de $\left\{a_{\rho}\right\}_{\rho<\lambda}$.

Todo corpo pseudocompleto (ou equivalentemente maximal mente completo) satisfaz o Lema de Hensel. A demonstração disto pode ser feita de forma similar à da demonstração do teorema I4.1 (de que todo corpo completo satisfaz o Lema de Hense1) que extraimos do livro de Koppermann, onde ele na re alidade demonstra que todo corpo pseudocompleto satisfaz o Lèma de Hense1, usando a mais, argumentos de indução transfi 
nita para ordinais quaisquer. (veja [Kop] teorA.1.13 e exer. $4 \cdot 1.9)$.

Uma outra demonstração pode ser vista em [Rb]: a de que todo corpo maximalmente completo é completo por etapas (cor.1 do teor.3 do cap.D) e que todo corpo completo por etapas satis faz o Lema de Hensel (teor.4, cap.F) A definição de corpo completo por etapas (que não foi vista neste trabalho) e estes resultados, devem-se também ao Krul1.

Com este resultado, podemos aplicar a proposição I5.4, para obter o

TEOREMA 15.4 - Se $\langle\mathrm{K}, \mathrm{v}\rangle$ é um corpo finitamente ramificado e $\langle F, W\rangle$ e $\left\langle F^{\prime}, W^{\prime}\right\rangle$ são duas extensões imediatas maximais de $\langle K, v\rangle$, então existe um K-isomorfismo analítico entre $\langle F, W\rangle$ e $\left\langle F^{\prime}, W^{\prime}\right\rangle$.

\section{DEMONSTRAÇAOO}

Seja $D$ o conjunto de todos os K-isomorfismos analiticos entre subcorpos de $\langle\mathrm{F}, \mathrm{W}\rangle$ e $\left\langle\mathrm{F}^{\prime}, \mathrm{W}^{\prime}\right\rangle$

$D \neq \phi$, pois a aplicação idêntica de $K$ pertence a $D$; e dada uma cadeia de $D$ a reunião dos elementos da cadeia $\vec{e}$ um elemento de $D$.

Logo pelo Lema de Zorn, existe $\phi:\langle\mathrm{L}, \mathrm{U}\rangle \rightarrow\left\langle\mathrm{L}^{\prime}, \mathrm{U}^{\prime}\right\rangle$ elemento maximal de $D$.

Se $L \neq F$, seja $a \in F-L$. Como $\langle F, W\rangle$ é extensão imedia- 
ta de $\langle K, v\rangle$ e $\langle K, v\rangle c\langle L, u\rangle c\langle F, w\rangle$ e claro que $\langle F, w\rangle$ ë extensão imediata de $\langle L, u\rangle$ e portanto, pela proposiçãoI5.1, a $\vec{e}$ pseudolimite de alguma sequência $\left\{a_{\rho}\right\}_{\rho<\lambda}$ pseudoconvergente em L que näo admite pseudolimites em $L$.

Se $\left\{a_{\rho}\right\}_{\rho<\lambda} \quad \vec{e}$ transcendente em $L$, então $\left\{\phi\left(a_{\rho}\right)\right\}_{\rho<\lambda} \quad \vec{e}$ transcendente em $L^{\prime}$ e se $a^{\prime} \in F^{\prime} \vec{e}$ um pseudolimite de $\left\{\phi\left(a_{\rho}\right)\right\}_{\rho<\lambda}$ (existe algum $a^{\prime} \in F^{\prime}$, pois $\left\langle F^{\prime}, w^{\prime}\right\rangle \quad \bar{e} \operatorname{maximal}$ mente completo e portanto pseudocompleto), então por um argumento similar ao da parte (ii) do teorema 15.1, a aplica ção $\psi: L(a) \rightarrow L^{\prime}\left(a^{\prime}\right)$ definida por $\psi\left(\frac{f(a)}{g(a)}\right)=\frac{\phi \circ f\left(a^{\prime}\right)}{\phi \circ g\left(a^{\prime}\right)} \quad \vec{e}$ um isomorfismo analitico que extende $\phi$.

Se $\left\{a_{\rho}\right\}_{\rho<\lambda} \vec{e}$ algëbrica em L, então, pela proposiçãol 5.4 , existe $b \in F$ pseudolimite de $\left\{a_{\rho}\right\}_{\rho<\lambda}$ e tal que $q(b)=0$, onde $q$ é um polinômio minimal de $\left\{a_{\rho}\right\}_{\rho<\lambda}$ em $L$ (observe que como $\langle\mathrm{L}, \mathrm{u}\rangle \overrightarrow{\mathrm{e}}$ extensão imediata de $\langle\mathrm{K}, \mathrm{v}\rangle,\langle\mathrm{L}, \mathrm{u}\rangle$ tambẻm è finitamente ramificado). Por outro lado, como $\phi \vec{e}$ isomorfismo analîtico, a sequência $\left\{\phi\left(a_{\rho}\right)\right\}_{\rho<\lambda}$ é pseudoconvergente, algëbrica, sem pseudolimites em $L^{\prime}$ e com $q^{\prime}=\phi^{\circ} q$ um polinômio minimal em $L^{\prime}$. Logo existe $b^{\prime} \in F^{\prime}$ pseudolimite de $\left\{\phi\left(a_{\rho}\right)\right\}_{\rho<\lambda}$ tal que $q^{\prime}\left(b^{\prime}\right)=0$ e a aplicação $\psi: L(b)+L^{\prime}\left(b^{\prime}\right)$ definida por $\psi(f(b))=\psi \circ f\left(b^{\prime}\right)$ para $f \in L[X]$ com $\operatorname{gr}(f)<g r(q)$, serä um isomorfismo analitico que extende $\phi$.

Logo $L=F$, e por uma argumentação anäloga, $L^{\prime}=F^{\prime}$, e por 
tanto $\phi \bar{e}$ um K-isomorfismo analítico entre os corpos valorados $\langle\mathrm{F}, \mathrm{W}\rangle$ e $\left\langle\mathrm{F}^{\prime}, \mathrm{W}^{\prime}\right\rangle$. 


\section{CAPITULO II}

\section{UM TEOREMA DE ISOMORFISMO ENTRE CORPOS VALORADOS}

Para este capítulo vamos fixar de uma vez por todas a se guinte situação: $\langle K, v\rangle$ e $\left\langle K^{\prime}, v^{\prime}\right\rangle$ são dois corpos valorados, finitamente ramificados, com o mesmo indice de ramificação e, admitindo $x$-seçoes normalizadas $\pi$ e $\pi^{\prime}$ e tais que $v(K)=V^{\prime}\left(K^{\prime}\right)=G$ e $\bar{K}_{V} \simeq \bar{K}^{\prime}{ }_{V}{ }^{\prime}$. Onde, dizemos que uma $x$-seção $\pi$ de un corpo finitamente ramificado $\langle K, v\rangle \operatorname{com} e>0$ é normalizada se $\pi(v(p))=p$, para $p=\operatorname{car} \bar{K}_{V} \neq 0$; e que toda $x$-seção é normali zada se e $=0$ 。

Vamos também introduzir a seguinte nomenclatura:

DEFINICAO II. I

(i) Uma aplicação $\phi: E \rightarrow E^{\prime}$ se diz uma aplicação $x$-analitica entre $\langle K, v\rangle$ e $\left\langle K^{\prime}, v^{\prime}\right\rangle$ se E e E' são subcorpos de $K$ e $K^{\prime}$ tais que $v(E)=V^{\prime}\left(E^{\prime}\right)=H, \pi(H) c E, \quad \pi^{\prime}(H) C E^{\prime}, \bar{E}_{V \mid E}=$ $\bar{K}_{V}, \bar{E}_{V^{\prime}}^{\prime} \mid E^{\prime}=\widetilde{K}^{\prime}{ }_{V^{\prime}}$ e $\phi \bar{e}$ um isomorfismo $x$-analítico entre os corpos valorados $\langle\mathrm{E}, \mathrm{V} \mid \mathrm{E}\rangle$ e $\left\langle\mathrm{E}^{\prime}, \mathrm{V}^{\prime} \mid \mathrm{E}^{\prime}\right\rangle$ com $\mathrm{x}$-seções normalizadas $\pi \mid H$ e $\pi^{\prime} \mid H$. 
(ii) Uma aplicação $\phi: E \rightarrow \mathrm{E}^{\prime}$ è una aplicação pura entre $\langle\mathrm{K}, \mathrm{V}\rangle \mathrm{e}\left\langle\mathrm{K}^{\prime}, \mathrm{V}^{\prime}\right\rangle$ se ela é uma aplicação $\mathrm{x}$-analítica entre $\langle\mathrm{K}, \mathrm{V}\rangle$ e $\left\langle\mathrm{K}^{\prime}, \mathrm{V}^{\prime}\right\rangle$ e $\mathrm{V}(\mathrm{E})=\mathrm{V}^{\prime}\left(\mathrm{E}^{\prime}\right)=\mathrm{H}$ é um subgrupo puro e enumerävel (finito ou não) de G.

PROPOSICGAO II.I - Se $\langle K, V\rangle=\left\langle K^{\prime}, v^{\prime}\right\rangle$ satisfazem o lema de Hensel e $\mathrm{e}=0$ (isto $\overrightarrow{\mathrm{e}} \operatorname{car} \overline{\mathrm{K}}_{V}=\operatorname{car}_{\overline{\mathrm{K}}}{ }_{{ }^{\prime}{ }^{\prime}}=0$ ), então existe uma $\mathrm{a}$ plicação pura entre $\langle K, v\rangle$ e $\left\langle K^{\prime}, v^{\prime}\right\rangle$.

DEMOSTRAÇAO

Pelo teorema I3.1 se L e L' são subcorpos maximais de

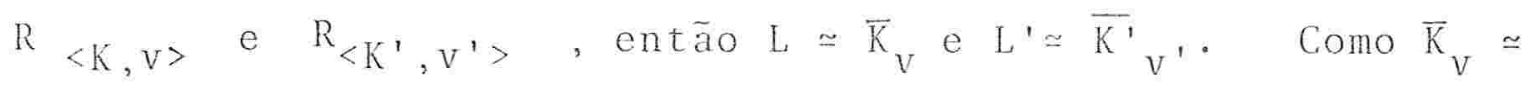
$\simeq \bar{K}^{\prime}{ }^{\prime}{ }^{\prime}$, segue que $L \simeq L^{\prime}$, e este isomorfismo entre $L$ e $L^{\prime}$ é uma aplicação pura entre $\langle K, V\rangle$ e $\left\langle K^{\prime}, V^{\prime}\right\rangle$, pois sendo $v(L)=v^{\prime}\left(L^{\prime}\right)=\{0\}$, todos os outros requisitos para aplicação pura são satisfeitos trivialmente.

Resta então mostrar que existem tais subcorpos maximais de $R_{\langle K, V\rangle}\left(e R_{\left\langle K^{\prime}, V^{\prime}>\right.}\right)$. E para isto ë suficiente mostrar que existe pelo menos um subcorpo de $K$ contido em $R_{<K, V>}$, pois então o lema de Zorn garantirä a existência de um subcorpo $\operatorname{maxima} 1$.

De fato como $\operatorname{car} \bar{K}_{V}=0$, segue que ${\overline{n \cdot I_{K}}}_{K}=n \cdot \overline{1}_{K} \neq \overline{0}_{\text {em }}$ em $K_{V}$ se $0 \neq n \in \mathbb{Z}$, donde $n \cdot 1_{K} \in U\left(R_{\langle K, v>}\right)$ e $\frac{m \cdot 1_{K}}{n \cdot 1_{K}} \in R_{<K, V>}$ para $m, n \in \mathbb{Z}(n \neq 0)$. Isto é: o subcorpo $Q \cdot 1_{K}$ de $K$ esta contido em $\mathrm{R}_{<K, V>}$. 
Vamos agora estudar o caso $0<\mathrm{e}<\infty$. LEMA II.1 - Seja F,W um corpo valorado com indice de ramificação $0<f<\infty$. O subgrupo $\mathbb{Z}_{\gamma}$, de $w(F)$, onde $\gamma_{1}=\min \{g \in W(F): 0<g\}$ (veja prop. I2.4) è un subgrupo puro de $W(F)$.

\section{DEMONSTRACAO}

$\mathbb{Z}_{Y}$, serä un subgrupo puro de $W(F)$ se de $g \in W(F)$ e $0 \neq n \in \mathbb{Z}$ com ng€ $\mathbb{Z}_{Y_{1}}$, segue que $g \in \mathbb{Z}_{Y_{1}}$. Não haverä prejuizo para a ge neralidade em se supor que $0 \leq \mathrm{g}$ e $\mathrm{n}>0$, para evitar-se proble mas com os sinais. Seja então n.g=m. $\gamma_{1} \in \mathbb{Z} \gamma_{1}$ nestas condições. Pelo algoritmo da divisão $m=q \cdot n+r$ com $0 \leq r<n$; donde $n g=q n \gamma_{1}+r \gamma_{1}$, ou $n\left(g-q \gamma_{1}\right)=r \gamma_{1}$. Daî segue que :

(i) $0 \leq \mathrm{g}-\mathrm{q} \gamma_{1}$, pois se $0>\mathrm{g}-\mathrm{q} \gamma_{1}$, então:

$$
0=n \cdot 0>n \cdot\left(g-q \gamma_{1}\right)=r \cdot \gamma_{1} \geqslant 0 \cdot \gamma_{1}=0 . \quad E
$$

(ii) $g-q \gamma_{1}<\gamma_{1}$, pois se $g-q \cdot \geq \gamma_{1}$, então:

$$
r \gamma_{1}=n\left(g-q \gamma_{1}\right)>r\left(g-q \gamma_{1}\right) \geq r \gamma_{1} \text {. }
$$

Logo $0 \leq \mathrm{g}-\mathrm{q} \gamma_{1}<\gamma_{1}$ e pela minimalidade de $\gamma_{1} \mathrm{em} w(F)$ segue que $0=g-q \gamma_{1}$ ou $g=q \cdot \gamma_{1} \in \mathbb{Z}_{\gamma_{1}}$.

PROPOSIÇÃO II.2 - Se $\langle K, V\rangle$ e $\left\langle K^{\prime}, V^{\prime}\right\rangle$ tem (alëm das condições jă impostas no início da seção) indice de ramificação $e=1$, satisfazem o lema de Hensel e são w-pseudocompletos, então existe uma aplicação pura entre eles.

\section{DEMONSTRAÇĀO}

Na seção 2 do 1 : Capítulo vimos como decompor uma valora 
ção $v$ em $v_{H}$ e $v / H$, onde $H \vec{e}$ um subgrupo convexo de $G=v(K)$ (Lema I2.3), e que o subgrupo $\mathbb{Z}_{\gamma_{1}}$ e um subgrupo convexo de G (prop. I2.4).

A aplicação que a $n \in \mathbb{Z}$ associa $n \cdot \gamma_{1} \in G$ é um monomorfismo do grupo $\mathbb{Z}$ no grupo G que presenrva a ordem e alêm disso leva o mínimo dos elementos positivos de $\mathbb{Z}$ no mínimo dos elementos positivos de $G$ cobserve que isto não ê necessariamente consequência do fato dele ser um monomorfismo que preserva ordem). Vamos então identificar $\mathbb{Z}$ com sua imagem que é precisa mente $\mathbb{Z} \gamma_{1}$ identificando 1 com $\gamma_{1}$. Como $e=1$, segue também que $v(p)=v^{\prime}(p)=1$ e como $\pi$ e $\pi^{\prime}$ são normalizadas, $\pi^{1}=\pi^{1}=p$ ou mais genericamente $\pi^{n}=\pi^{n}=p^{n}$ para $n \in \mathbb{Z}$ (lembramos que convenciona mos escrever $\pi^{g}$ ao invés de $\pi(g)$, escreverenos também $\pi^{H}$ para indicar a imagem $\pi(H)$ de um subconjunto $H$ de $G$ ).

Temos então:

$\left.<K, v_{\mathbb{Z}}, G / \mathbb{Z}\right\rangle$ onde $v_{\mathbb{Z}}(a)=v(a)+Z \in G / Z$.

e $\left\langle\bar{K} v_{\mathbb{Z}}, v / \mathbb{Z}, \mathbb{Z}\right\rangle$ onde $v / \mathbb{Z}\left(a+I_{<K, v_{\mathbb{Z}}}\right)=v(a) \in \mathbb{Z}$.

e sabemos que $\left(\overline{\mathrm{K}}_{\mathrm{v}_{\mathbb{Z}}}\right)_{V / \mathbb{Z}} \simeq \overline{\mathrm{K}}_{V} \quad\left(\right.$ Iema I2.4) e que $\operatorname{car} \overline{\mathrm{K}}_{v_{\mathbb{Z}}}=0$ (prop. I2.4) e as mesmas coisas valendo para $\left\langle K^{\prime}, V^{\prime}\right\rangle$. Mostrando que:

(1) $\left\langle K, V_{\mathbb{Z}}, G / \mathbb{Z}\right\rangle$ e $\left\langle K^{\prime}, V^{\prime}{ }_{\mathbb{Z}}, G / \mathbb{Z}\right\rangle$ satisfazem 0 lema de Hensel e (2) $\left\langle\bar{K}{ }_{V_{\mathbb{Z}}}, v / \mathbb{Z}, \mathbb{Z}\right\rangle$ e $\left\langle\bar{K}^{\prime}{ }_{V^{\prime}}{ }_{\mathbb{Z}}, v^{\prime} / \mathbb{Z}, \mathbb{Z}\right\rangle$ são completos

teremos que de (1) seguirä que existem subcorpos L e L' de K e K' 
isomorfos a $\bar{K}_{V_{\mathbb{Z}}}$ e $\bar{K}^{\prime}{ }_{V^{\prime}}{ }_{\mathbb{Z}}$ e cujos elementos não nulos estão em $\mathrm{U}\left(\mathrm{R}_{\left\langle\mathrm{K}, \mathrm{v}_{\mathbb{Z}}>\right.}\right)$ e $\mathrm{U}\left(\mathrm{R}_{\left\langle\mathrm{K}^{\prime}, \mathrm{V}^{\prime} \mathbb{Z}^{>}\right.}\right)$. e portanto tem valoraçoes pertencen tes a $\mathbb{Z}$ (Iema I2.3). Enquanto que de (2) seguira que $\left\langle\bar{K}_{v_{\mathbb{Z}^{m}}}, v / Z, Z\right.$, e $\left\langle\bar{K}{ }^{\prime}{ }_{V^{\prime}}, v^{\prime} / \mathbb{Z}, \mathbb{Z}\right\rangle$ são analiticamente isomorfos pois são corpos valorados completos de característica zero com corpos de clas

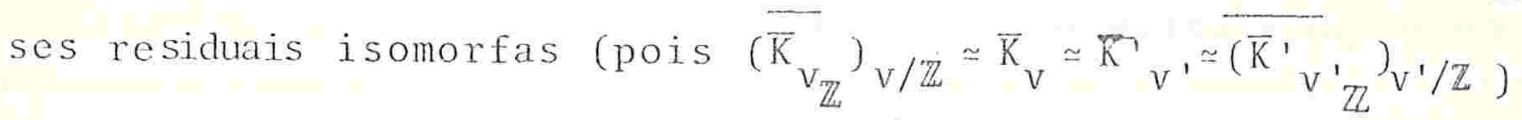
de característica $p \neq 0$ e $v / \mathbb{Z}(p)=v^{\prime} / \mathbb{Z}(p)=1$. (veja os comen tärios do final da $4^{\text {a }}$ seção do $1^{\text {? }}$ Capítulo).

Temos então a seguinte situação:

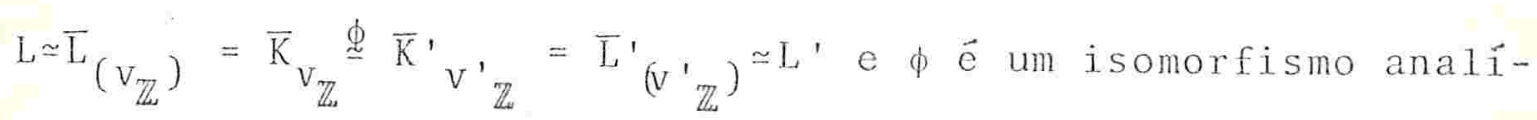
tico, isto è: $v / \mathbb{Z}\left(a+I_{<K, v_{\mathbb{Z}}}\right)=v^{1} / \mathbb{Z}\left(\phi\left(a+I_{<K, v_{\mathbb{Z}}>}\right)\right)$; e temos um isomorfismo $\psi: L \rightarrow L^{\prime}$ que a aEL associa $\psi(a) E L^{\prime}$ onde $\psi(a) \in L^{\prime}$ é o ünico elemento de $L^{\prime}$ tal que $\phi\left(a+I_{<K, v_{\mathbb{Z}}}\right)=\psi(a)+I_{<K^{\prime}, v^{\prime}{ }_{\mathbb{Z}^{\prime}}{ }}$ (este isomorfismo entre os corpos L e L' è simplesmente a com posição de todos os isomorfismosescritos acima).

Este isomorfismo $\vec{e}$ analítico pois:

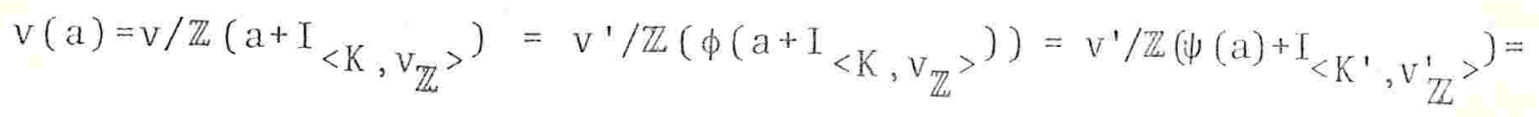
$=v^{\prime}(\psi(\mathrm{a}))$ e como $\pi^{\mathbb{Z}}=\left\{p^{n}: n \in \mathbb{Z}\right\} \subset Q \subset L$ e $\pi^{\prime} \mathbb{Z}^{n}=\left\{p^{n}: n \in \mathbb{Z}\right\} \subset Q \subset L^{\prime}$ (pois $Q \vec{e}$ o corpo primo de $L$ e $\left.L^{\prime}\right)$ e $\psi / Q \vec{e}$ a identidade temos tambëm:

$\psi\left(\pi^{n}\right)=\psi\left(p^{n}\right)=p^{n}=\pi^{n}$ para todo $n \in \mathbb{Z}$ e portanto $\psi \vec{e} x$-analítico. 
Finalmente pelo Lema anterior $v(L)=V^{\prime}\left(L^{\prime}\right)=\mathbb{Z}$ (pois já tínha-l mos que $v(L) \subset \mathbb{Z}$ e $V^{\prime}\left(L^{\prime}\right) \subset \mathbb{Z}$ uma vez que $L^{*} \subset U\left(R_{<K, v_{\mathbb{Z}}>}\right) \quad$ e $L^{\prime *} \subset U\left(R_{\left\langle K^{\prime}, v_{\mathbb{Z}^{\prime}}>\right.}\right)$ pelo Lema I2.3, e é claro que $\mathbb{Z} \subset \mathrm{V}(\mathrm{L})$ e $\mathbb{Z} \subset V^{\prime}\left(L^{\prime}\right)$ pois $p \in L, p \in L^{\prime}$ e $v\left(p^{n}\right)=v^{\prime}\left(p^{n}\right)=n$ para $\left.n \in \mathbb{Z}\right) \quad \vec{e}$ um subgrupo puro e enumerável de $G$ e portanto $\psi: L \rightarrow L^{\prime} \vec{e}$ uma apli cação pura entre $\langle K, v\rangle$ e $\left\langle K^{\prime}, v^{\prime}\right\rangle$.

Falta então demonstrar as afirmações (1) e (2). (1): Para demonstrar que $\left\langle K, v_{\mathbb{Z}}, G / \mathbb{Z}\right\rangle$ satisfaz o Lema de Hensel. teremos que recorrer a uns resultados sobre prolongamentos de valoraçöesa extensões algébricas do Capítilo F do [Rb]. o pri meiro é a equivalência entre o fato de um corpo valorado satisfazer o Lema de Hensel e o fato dele admitir um ünico prolongamento a cada extensão algébrica que jả havíamos cnunciado no inîcio da $3^{a}$. seção do $1^{\circ}$ capítulo. o segundo é o seguin te: (proposição 4 do cap. F de $[R b]$ ): "Se $\bar{K}$ é uma extensão algëbrica de $K, P$ um ideal primo de $R_{<K}, v>, v_{P}$ a valoração as sociada a $\mathrm{P}$ (que é $\mathrm{v}_{\mathrm{H}}$ onde $\mathrm{H}$ é o subgrupo convexo de $\mathrm{G}$ associado a P, veja prop. I2.3) e w uma extensão de $v_{p}$ a $\tilde{K}$, então existe uma extensão $\tilde{v}$ de $v$ a $\tilde{K}$ tal que w $\bar{e} \tilde{v} \tilde{p}$ onde $\tilde{p} e \bar{e}$ unico ideal primo de $\mathrm{R}_{<\tilde{K}, \tilde{v}>}$ tal que $\tilde{\mathrm{P}}_{\mathrm{R}} \mathrm{R}_{<\mathrm{K}, \mathrm{V}\rangle}=\mathrm{P}$. ". Deste resultado segue que se $v$ admite uma ünica extensão a $\tilde{K}$, então $v_{p}$ (e portanto $v_{H}$ ) também admite una única a $\tilde{K}$; que combinado com o primeiro resultado nos diz que se $\langle K, v, G\rangle$ satisfaz o Lema de Hense1, então $\left.<K, v_{H}, G / H\right\rangle$ também satisfaz o Lema de Hensel pa- 
ra todo $H$ subgrupo convexo de $G$.

(2) Seja $\bar{a}_{n}=a_{n}+I_{<K, v_{\mathbb{Z}}>}$ uma sequência de Cauchy de $<\overline{\mathrm{K}}_{v_{\mathbb{Z}}}$, $v / \mathbb{Z}, \mathbb{Z}>$. Então $v / \mathbb{Z}\left(\bar{a}_{m}-\bar{a}_{n}\right)+\infty \quad(v e j a$ o início da $\$ 4$ do cap.1) ou $v\left(a_{m}-a_{n}\right) \rightarrow \infty$ e portanto $v\left(a_{n+1}-a_{n}\right) \rightarrow \infty$. Disto segue que pode mos extrair uma subsequência $\left\{a_{n_{k}}\right\}$ de $\left\{a_{n}\right\}$ tal que $v\left(a_{n_{k+1}}\right.$ $-a_{n_{k}}$ ) seja crescente (estritamente), e portanto $\left\{a_{n_{k}}\right\} \vec{e}$ uma sequência pseudoconvergente em K (def.I5.1); e como $\langle K, v\rangle \vec{e}$ $\omega$-pseudocompleto $\left\{a_{n_{k}}\right\}$ admite um pseudolimite a€K. Se zero $\vec{e}$ um pseudolimite de $\left\{a_{n_{k}}\right\}$ então $\overline{0}=0+I_{\left\langle K, v_{\mathbb{Z}^{2}}\right.}$ è um limite de $\left\{\bar{a}_{n}\right\}$. Senão, pelo comențärio após o lema 15.3 , existe $k_{0}$ tal que $v(a)=v\left(a_{n_{k}}\right)$ para $k \geq k_{0}$ e portanto $v(a) \in \mathbb{Z}$, donde $a \in R_{<K, v_{\mathbb{Z}}}$ > e portanto $\overline{\mathrm{a}}=\mathrm{a}+\mathrm{I}_{<\mathrm{K}, v_{\mathbb{Z}}>} \in \overline{\mathrm{K}}_{V_{\mathbb{Z}}} \overrightarrow{\mathrm{e}}$ um limite de $\left\{\overline{\mathrm{a}}_{\mathrm{n}}\right\}$.

Uma vez conseguidas aplicações puras entre $\langle K, v\rangle \mathrm{e}\left\langle\mathrm{K}^{\prime}, v^{\prime}\right\rangle$ nosso trabalho agora será o de extendê-las atẻ chegarmos a um isomorfismo entre os dois corpos. Num primeiro passo (atê prop. II.4) extenderamos as aplicações puras a corpos com grupos de valores maiores que os dos corpos de partida; e apos isso (proposição II.5) as extenderemos a extensões imediatas dos corpos iniciais, contendo algum elemento novo prefixado. Observe que sè $\phi: E \rightarrow E^{\prime} \vec{e}$ uma aplicação pura entre $\left\langle K, v>e<K^{\prime}, v^{\prime}\right\rangle$,

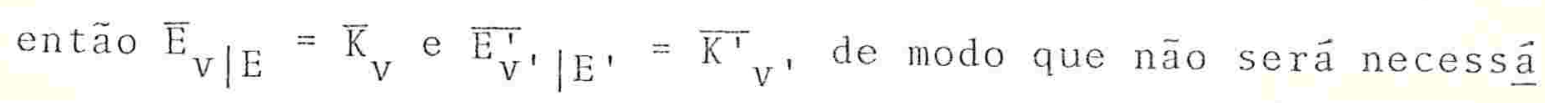
rio extender o corpo das classes residuais nas extensões das aplicações puras.

LEMA II.2 - Seja $\phi: E \rightarrow E^{\prime}$ uma aplicação pura entre $\langle K, v\rangle$ e $\left\langle K^{\prime}, v^{\prime}\right\rangle$ 
e $\alpha E G-v(E)$. Então existe una aplicação $x$-analítica $\psi: E\left(\pi^{\alpha}\right) \rightarrow E^{\prime}\left(\pi^{\prime \alpha}\right)$ entre $\langle K, v\rangle$ e $\left\langle K^{\prime}, v^{\prime}\right\rangle$ extendendo $\phi$.

\section{DEMONSTRAÇÃO}

$v(E)=v^{\prime}\left(E^{\prime}\right)$ é um subgrupo puro de $G$ e $v\left(\pi^{\alpha}\right)=\alpha . E v(E) \quad$ e $V^{\prime}\left(\pi^{\alpha}\right)=\alpha \notin V^{\prime}\left(E^{\prime}\right)$. Logo, pelo $3^{\circ}$ corolário da proposição I3.1, $\alpha$ e $\alpha^{\prime}$ são transcendentes sobre E e E' respectivamente. E portanto a aplicação $\psi: E\left(\pi^{\alpha}\right) \rightarrow E^{\prime}\left(\pi^{\prime \alpha}\right)$ definida por $\psi\left(f\left(\pi^{\alpha}\right) /\right.$ $\left./ \cdot g\left(\pi^{\alpha}\right)\right)=\phi f\left(\pi^{\prime \alpha}\right) / \phi \circ f\left(\pi^{\prime \alpha}\right)$ para $f, g \in E[X] \quad g \neq 0$ e um isomorfis mo (algébrico) entre os dois corpos que extende $\phi$ (onde $\phi \circ f=\sum_{i=0} \phi\left(c_{i}\right) X^{i} \in E^{\prime}[X]$ para $\left.f=\sum_{i=0}^{n} c_{i} X^{i} \in E[X]\right)$.

Seja $0 \neq \mathrm{f}=\sum_{i=0}^{n} c_{i} X^{i} \in E[X]$. Pelo corolärio citado acima: $v\left(f\left(\pi^{\alpha}\right)\right)=v\left(\sum_{i=0}^{n} c_{i} \pi^{i \alpha}\right)=\min \left\{v\left(c_{i}\right)+i \cdot \alpha: 0 \leq i<n\right\}=\min \left\{v^{\prime}\left(\phi\left(c_{i}\right)\right)+i \alpha: 0 \leq i<n\right\}=$ $=v^{\prime}\left(\sum_{i=0}^{n} \phi\left(c_{i}\right) \pi^{\prime i \alpha}\right)=v^{\prime}\left(\phi f\left(\pi^{\prime \alpha}\right)\right)=v^{\prime}\left(\psi\left(f\left(\pi^{\alpha}\right)\right)\right)$ o que mostra que $\psi$ é um isomorfismo analítico, uma vez que: $v\left(E\left(\pi^{\alpha}\right)\right)=V(E) \oplus \mathbb{Z} \alpha=V^{\prime}\left(E^{\prime}\right) \oplus \mathbb{Z} \alpha=V^{\prime}\left(E^{\prime}\left(\pi^{\prime \alpha}\right)\right)=H$ Se $\gamma \in v(E)$ e $n \in Z$, então $\pi^{\gamma+n \alpha}=\pi^{\gamma} \cdot \pi^{n \alpha}=\pi^{\gamma} \cdot\left(\pi^{\alpha}\right)^{n} \in E\left(\pi^{\alpha}\right)$ pois $\pi^{\gamma} \in E$ e portanto $\pi^{H} \subset E\left(\pi^{\alpha}\right)$ e analogamente $\pi^{, H} \subset E^{\prime}\left(\pi^{1}{ }^{\alpha}\right)$, donde $\pi \mid H$ e $\pi^{\prime} \mid H$ são $x$-seçöes de $\left\langle\mathrm{E}\left(\pi^{\alpha}\right), v \mid E\left(\pi^{\alpha}\right)\right\rangle$ e $\left\langle\mathrm{E}^{\prime}\left(\pi^{, \alpha}\right), \mathrm{v}^{\prime} \mid \mathrm{E}^{\prime}\left(\pi^{, \alpha}\right)\right\rangle$ respectivamente.

Finalmente se $\gamma \in v(E)$ e $n \in Z$, entäo: 
$\psi\left(\pi^{\gamma+n \alpha}\right)=\psi\left(\pi^{\gamma} \cdot\left(\pi^{\alpha}\right)^{n}\right)=\psi\left(f\left(\pi^{\alpha}\right)\right)=\phi \circ f\left(\pi^{\alpha}\right)=\phi\left(\pi^{\gamma}\right) \cdot(\pi, \alpha)^{n}=\pi, \gamma, \pi, n \alpha=\pi, \gamma+n \alpha$

(onde $f=\pi^{\gamma} \cdot X^{n} \in E[X]$ e $\phi\left(\pi^{\gamma}\right)=\pi{ }^{\gamma}$ pois $\phi \dot{e} x$-analítica).

Logo $\psi \vec{e} x$-analítica. E como $\left.\overline{\mathrm{E}}_{V} \subset \overline{\mathrm{E}(\pi(\alpha)}\right)_{V} \subset \overline{\mathrm{K}}_{V}, \overline{\mathrm{E}(\pi(\alpha))_{V}}=\overline{\mathrm{K}}_{V} \mathrm{e}$ analogamente $\overline{\mathrm{E}^{\prime}\left(\pi^{1 \alpha}\right)_{V^{\prime}}}=\bar{K}^{\prime}{ }_{V^{\prime}}$ de modo que $\psi$ satisfaz todas as condições para ser uma aplicação $x$-analítica entre $<K, v>\quad e$ $\left\langle K^{\prime}, V^{\prime}\right\rangle$.

LEMA II. 3 - Sejam $\phi: E \rightarrow E^{\prime}$ uma aplicação $x$-analítica entre $\langle K, v\rangle$ e $\left\langle K^{\prime}, v^{\prime}\right\rangle$ e $\beta \in G-V(E)$ tal que $p \beta E v(E)$ para algum $p$ inteiro primo. Então existe uma aplicação $x$-analîtica $\psi: E\left(\pi^{\beta}\right) \rightarrow E^{\prime}\left(\pi^{\prime}{ }^{\beta}\right)$ entre $\langle K, v\rangle$ e $\left\langle K^{\prime}, V^{\prime}\right\rangle$ extendendo $\phi$.

\section{DEMONSTRACAO}

Pelo Corolário 4 da proposição I3.1, $x^{p_{-\pi}} p^{p}$ e $x^{p}-\pi{ }^{p B}$ são os polinômios minimais de $\pi^{\beta}$ e $\pi^{\beta} \beta$ sobre $E$ e $E^{\prime}$ respectivamente. Logo a aplicação $\psi: E\left(\pi^{\beta}\right) \rightarrow E^{\prime}\left(\pi^{\beta}\right)$ definida por $\quad \psi\left(f\left(\pi^{\beta}\right)\right)=$ $=\phi f\left(\pi^{\beta}\right)$ para $f \in E|X|$ com gre<p, é um isomorfismo (algëbrico) entre os dois corpos.

E como aqui tambëm vale:

$v\left(f\left(\pi^{\beta}\right)\right)=v\left(\sum_{i=0}^{r} a_{i} \pi^{i \beta}\right)=\min \left\{v\left(a_{i}\right)+i=\beta: 0 \leq i \leq r\right\}$, segue por una ar gumentação similar a do lema anterior que $\psi$ é uma aplicação $x$-analitica entre $\langle K, v\rangle$ e $\left\langle K^{\prime}, v^{\prime}\right\rangle$.

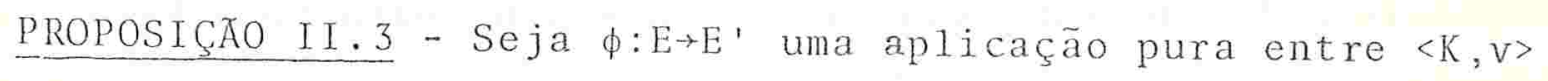
$\mathrm{e}\left\langle\mathrm{K}^{\prime}, \mathrm{v}^{\prime}\right\rangle, \alpha \in G-\mathrm{V}(\mathrm{E}), \mathrm{H}=\mathrm{v}\left(\mathrm{E}\left(\pi^{\alpha}\right)\right)$ e $\tilde{\mathrm{H}}=\tilde{\mathrm{H}}_{\mathrm{G}}$.

Então existe $\left.\psi: E\left(\pi^{\tilde{H}}\right) \rightarrow E^{\prime} \pi^{\prime}, \tilde{H}\right)$ aplicação pura entre $\quad<K, v>$ e 
e $\left\langle K^{\prime}, v^{\prime}\right\rangle$, e $v\left(E\left(\pi^{\tilde{H}}\right)\right)=v^{\prime}\left(E^{\prime}\left(\pi^{\prime} \tilde{H}\right)\right)=\tilde{H}$.

\section{DEMONSTRAÇATO}

Por definição de aplicação pura, v(E) é enumerävel e portanto $H=v\left(E\left(\pi^{\alpha}\right)\right)=v(E) \oplus \mathbb{Z} \alpha(\operatorname{cor} .3$ da prop. I3.1) também $\vec{e}$ enumerável.

$\widetilde{H}=\{g \in G$ :ng $\in H$ para algum $0 \neq n \in \mathbb{Z}\}$. Para cada $h \in H$ e $0 \neq n \in \mathbb{Z}$ exis te no máximo um $g \in G$ tal que $n \cdot g=h \quad\left(n g_{l}=n g_{2}\right.$ implica $g_{i}=g_{2}$ pois G é sem torção uma vez que é ordenado). Logo para cada $0 \neq n \in \mathbb{Z}$ o conjunto $A_{n}=\{g \in G: n g \in H\}$ e injetâvel em $H$ e portanto enume ráve1. Como $\tilde{H}=U_{A}$ segue que $\tilde{H} \vec{e}$ enumerâvel.

$$
n \in \mathbb{Z}
$$

Seja $\tilde{H}=\left\{h_{1}, h_{2} \ldots\right\}$ uma enumeração de $\tilde{H}$ e seja $\left\{H_{n}\right\}_{n \in \mathbb{N}}$ uma sequência de subgrupos de $\tilde{H}$, construida indutivamente, como segue :

$$
H_{0}=H=V\left(E\left(\pi^{\alpha}\right)\right)=V^{\prime}\left(E^{\prime}\left(\pi^{\alpha}\right)\right) .
$$

Supondo construido $H \subset H_{n} \subset \tilde{H}$; se $H_{n}=\tilde{H}$, então $H_{n+1}=\tilde{H}$. Se $\mathrm{H}_{\mathrm{n}} \not \tilde{\mathrm{H}}$, então seja $\ell=\min \left\{\mathrm{k}>0: \mathrm{h}_{\mathrm{k}} \mathrm{eH}_{\mathrm{n}}\right\}$. Como existe algum inteiro $m>0$ tal que $m \cdot h_{\ell} \in H \subset H_{n}$, seja $r=m i n\left\{m>0: m \cdot h_{\ell} \in H_{n}\right\}$ e seja $p>1$ o menor primo que divide $r$. $H_{n+1}$ será o subgrupo de $\tilde{H}$ (e portanto tambëm de G) gerado por $H_{n}$ e $\frac{r_{p}}{p} h_{\ell}=\beta_{n}$.

$\tilde{H}=\underset{n \in \mathbb{N}}{U} H_{n}$ : de fato supondo que $h_{k} \in \underset{n \in \mathbb{N}}{U} H_{n}$ para todo $k<\ell$, seja $n_{0}=\min \left\{n \in \mathbb{N}: h_{k} \in H_{n}\right.$ para todo $\left.k<\ell\right\}$. Se $h_{\ell} \in H_{n_{0}}$, então $\ell=\min \left\{k>0: h_{k} \in H_{n_{0}}\right\}$ e se $\min \left\{m>0: m . h_{\ell} \in H_{n}\right\}=r=p_{1}^{\nu_{i}} \ldots p_{s}^{\nu_{s}}$, 
então após, no máximo $v_{1}+\ldots+v_{s}$ construções, $h_{\ell} \in H_{n+\nu_{1}+\ldots+v_{s}}$ uma vez que apös cada construção o $r$ correspondente diminui de um fator primo. Logo da hipótese que $h_{k} \in \underset{n \in \mathbb{N}}{U} H_{n}$ para $k<\ell$ segue que $h_{\ell} \underset{n \in \mathbb{N}}{U} H_{n}$ e portanto $\tilde{H}=\left\{h_{1}, h_{2}, \ldots\right\}=\underset{n \in \mathbb{N}}{U} H_{n}\left(j a \dot{a} q u e \tilde{H} \underset{n \in \mathbb{N}}{\sim} \underset{n}{U} H_{n}\right)$.

Logo $\underset{n \in \mathbb{H}}{\tilde{U}} \mathrm{U}_{n}$ onde para cada nEIN, $H_{n+1} \vec{e}$ o subgrupo de $\tilde{H}$ gerado por $H_{n}$ e $\beta_{n}$, onde $\beta_{n} \& H_{n}$ e $p \cdot \beta_{n}=p \frac{r}{p} h_{\ell}=r \cdot h_{\ell} \cdot \epsilon^{\cdot} H_{n} \quad$ para algum primo p (ou este processo ocorre apenas até algum n ${ }_{0}$ e $\mathrm{H}_{\mathrm{n}}=\tilde{\mathrm{H}}$ para $\mathrm{n} \geq \mathrm{n}_{0}$ ).

Sejam: $E_{0}=E\left(\pi^{\alpha}\right), E_{0}^{\prime}=E^{\prime}\left(\pi^{\alpha}\right)$.

$$
E_{n+1}=E_{n}\left(\pi^{\beta n}\right), E^{\prime}{ }_{n+1}=E^{\prime}{ }_{n}\left(\pi, B^{B}\right)
$$

(ou $E_{n}=E_{n_{0}}, E^{\prime}{ }_{n}=E^{\prime} n_{0}$ para $n \geq n_{0}$ se $\left.H_{n_{0}}=\tilde{H}\right)$.

Então $v\left(E_{0}\right)=v^{\prime}\left(E_{0}^{\prime}\right)=H_{0}$ e para $n>0$, pelo Corolário 4 da proposição 13.1 e por indução, $v\left(E_{n}\right)=V^{\prime}\left(E^{\prime}{ }_{n}\right)=H_{n} \quad$ e $\pi^{H_{n} \in E_{n}}$ e $\pi^{, H} n \subset E^{\prime}{ }_{n}$.

Logo existe, pelo lema II.2. $\psi_{0}: E_{0} \rightarrow E_{0}^{\prime}$ aplicação $x$-analítica extendendo $\phi$ e supondo que $\psi_{n}: E_{n} \rightarrow E^{\prime}{ }_{n} \vec{e}$ uma aplicação $x$-analítica de $\langle K, v\rangle$ e $\left\langle K^{\prime}, v^{\prime}\right\rangle$, então pelo lema II.3 existe $\psi_{n+1}: E_{n+1} \rightarrow E_{n+1}^{\prime}$ aplicação $x$-analítica extendendo $\psi_{n}$. E portanto existe uma sequência crescente $\left\{\psi_{n}: E_{n} \rightarrow E^{\prime}{ }_{n}\right\}_{n \in \mathbb{N}}$ de apli cações $x$-analíticas com $\psi_{0}$ extendendo $\psi$.

Sejam: $E_{\omega}=\underset{n \in \mathbb{N}}{U} E_{n}, E^{\prime} \omega_{n \in \mathbb{N}}^{U} E^{\prime}{ }_{n}, \psi_{\omega}=\underset{n \in \mathbb{N}}{U} \psi_{n}$. 
Então $\psi_{\omega}$ é um isomorfismo (algébrico) entre os corpos $E_{\omega}$ e $E_{\omega}^{\prime}$ e como $v\left(E_{\omega}\right)=v\left(u E_{n}\right)=u v\left(E_{n}\right)=u H_{n}=\tilde{H}=v^{\prime}\left(E_{\omega}^{\prime}\right) \quad$ e $\pi^{\tilde{H}}=u \pi^{H} \subset E_{n}=E_{\omega}$ e $\pi \tilde{H}_{\subset} \mathrm{E}_{\omega}^{\prime} \quad$ segue que $\psi_{\omega} \bar{e}$ uma aplicação $x$-analítica entre $\langle K, V\rangle$ e $\left\langle K^{\prime}, V^{\prime}\right\rangle$ e mais ainda uma aplicaçäo para entre $\langle K, v$, e $\left\langle K^{\prime}, v^{\prime}\right\rangle$ pois $\tilde{H}=v\left(E_{\omega}\right)=v^{\prime}\left(E_{\omega}^{\prime}\right) \vec{e}$ um subgrupo puro e enumera $\vec{a}-$ vel de G.

Finalmente $E_{\omega}=E\left(\pi^{\tilde{H}}\right)$ e $E_{\omega}^{\prime}=E^{\prime}\left(\pi, \pi^{H}\right)$ pois de $\pi^{\tilde{H}_{c}} \subset E_{\omega}$ segue $E\left(\pi^{\tilde{H}}\right) \subset E_{\omega}$ e reciprocamente, por indução, se $E_{n} \in E\left(\pi^{\tilde{H}}\right)$ então $E_{n+1}=E_{n}\left(\pi^{\beta} n\right) \subset E\left(\pi^{\tilde{H}}\right)\left(\pi^{\beta} n\right)=E\left(\pi^{\tilde{H}}\right)\left(\right.$ e é claro que $\left.E=E\left(\pi^{\alpha}\right) \subset E\left(\pi^{\tilde{H}}\right)\right)$. Logo $E_{\omega}=\underset{n C \mathbb{N}}{U} E_{n} \subset E\left(\pi^{\tilde{H}}\right)$. Analogamente $E_{\omega}^{\prime}=E^{\prime}(\pi, \tilde{H})$ e portanto $\psi_{\omega}: E\left(\pi^{\tilde{H}}\right) \rightarrow E^{\prime}\left(\pi^{\prime} \tilde{H}\right) \quad \vec{e}$ uma aplicação pura entre $\langle K, v\rangle$ e $\left\langle K^{\prime}, v^{\prime}\right\rangle$ que extende $\phi$.

PROPOSIÇÃO II.4 - Seja $\phi: E \rightarrow E^{\prime}$ uma aplicação pura entre $\langle K, v\rangle$ e $\left\langle K^{\prime}, v^{\prime}\right\rangle$ e seja a€K. Então:

(i) existe uma aplicação pura $\psi: E\left(\pi{ }^{2}\right) \rightarrow E^{\prime}\left(\pi,{ }^{\prime}{ }^{\prime}\right)$ entre $\langle K, v\rangle$ e $\left\langle K^{\prime}, v^{\prime}\right\rangle$ extendendo $\phi$, onde $H=v(E(a)) \quad\left(e \tilde{H}=\tilde{H}_{G}\right) ;$ e $\quad V\left(E\left(\pi^{\tilde{H}}\right)\right)=$ $=v^{\prime}\left(E^{\prime}(\pi, \tilde{H})\right)=\tilde{H}$.

(ii) existe uma ap1icação pura $\psi: F \rightarrow F^{\prime}$ entre $\langle K, v\rangle \quad$ e $\left\langle K^{\prime}, V^{\prime}\right\rangle$ extendendo $\phi, \operatorname{com} v(F(a))=V(F)$.

\section{DEMONSTRACAO}

(i) Se $H=v(E(a))=v(E)$, então $\tilde{H}=v(E)$ e $\pi^{\tilde{H}}$ E pois $\phi$ é pu ra. Logo $E\left(\pi^{\tilde{H}}\right)=E, E^{\prime}\left(\pi^{\prime H}\right)=E^{\prime}$ e $\psi=\phi$ è a aplicação procurada. 
Se $H=v(E(a)) \neq v(E)$, então existe ceE(a) tal que $v(c) \notin v(E)$. De cEE(a) e cEE (pois $v(c) \notin V(E))$ segue que a é algébrico sobre $E(c)$. De fato: $\operatorname{cEE}(a)$ implica que $c=\frac{f(a)}{g(a)}$ com $f, g \in E[X]$, donde $h(a)=f(a)-c \cdot g(a)=0$ com $h=f-c \cdot g \in E(c)[X]$ e $h \neq 0$ senão cEE. Logo E(a) c $\widetilde{E(c)_{K}}$ e, pela proposição $I 3.1, H=v(E(a)) c$ $c v(\widetilde{E(c)})_{K} \subset \tilde{v}(E(c))_{G}$ e como $\tilde{v}(E(c))_{G}$ é puro em $G$ segue que $\tilde{H} \subset \widetilde{V}(E(c))_{G}$.

Também de cEE(a) segue $E(c) \subset E(a), v(E(c)) \subset v(E(a))=H$ e $\tilde{v}(E(c))_{G} c \tilde{H}_{G}=\tilde{H} \cdot \operatorname{Logo} \tilde{H}=\tilde{V}(E(c))_{G}$.

Por outro lado como $v(C) \& v(E)$ e $v(E)$ é puro em $G$, segue pelo 3: Corolário da proposição I3.1, que $v(E(c))=v(E) \oplus \mathbb{Z} \cdot v(c)$. Se $\alpha=v(c)$, então $v\left(\pi^{\alpha}\right)=\alpha=v(c) \notin v(E)$ e pelo mesmo resultado $v\left(E\left(\pi^{\alpha}\right)\right)=v(E) \oplus \mathbb{Z} \cdot \alpha=v(E) \oplus \mathbb{Z} \cdot v(C)=v(E(C))$.

Donde $\tilde{H}=\tilde{v}(E(c))_{G}=\tilde{v}\left(E\left(\pi^{\alpha}\right)\right)_{G}$ e pela proposição anterior, existe $\psi: E\left(\pi^{\tilde{H}}\right) \rightarrow E^{\prime}\left(\pi^{,} \tilde{H}\right)$ aplicação pura extendendo $\phi ;$ e $v\left(E\left(\pi^{\tilde{H}}\right)\right)=$ $=v^{\prime}\left(E^{\prime}\left(\pi, \tilde{H}^{\prime}\right)\right)=\tilde{v}\left(E\left(\pi^{\alpha}\right)\right)_{G}=\tilde{H}$.

(ii) $\operatorname{Sejam} E_{0}=E, E_{0}^{\prime}=E^{\prime}$ e $\psi_{0}=\phi$. Supondo que foram cons truidos indutivamente $E_{n}, E_{n}^{\prime}$ e $\psi_{n}: E_{n} \rightarrow E_{n}^{\prime}$ aplicação pura ex tendendo $\phi$, sejam $H_{n+1}=v\left(E_{n}(a)\right), \tilde{H}_{n+1}=\left(\tilde{H}_{n+1}\right)_{G}, E_{n+1}=E_{n}\left(\pi^{H} n+j\right.$, $E_{n+1}^{\prime}=E_{n}^{\prime}\left(\pi, \tilde{H}_{n+1}\right)$ e $\psi_{n+1}: E_{n+1} \rightarrow E_{n+1}^{\prime}$ a aplicação pura que ex tende $\psi_{n}$ dada pelo îtem (i) desta proposição.

A sequência $\left\{\psi_{n}: E_{n} \rightarrow E_{n}^{\prime}\right\}_{n \in \mathbb{N}}$ assim construida éuma sequên cia crescente de aplicações puras com $v\left(E_{n}\right)=V^{\prime}\left(E_{n}^{\prime}\right)=\tilde{H}_{n} \quad(f a-$ zendo $\tilde{H}_{0}=H_{0}=v(E)=v^{\prime}\left(E_{0}^{\prime}\right)$ vale a igualdade para todo $\left.n \in \mathbb{N}\right)$. 
Sejam $F=\underset{n \in \mathbb{N}}{U} E_{n}, F^{\prime}=\underset{n \in \mathbb{N}}{U} E^{\prime}{ }_{n}$ e $\psi=\underset{n \in \mathbb{N}}{U} \psi_{n}: F \rightarrow F^{\prime}$. E fäcil ver que $\psi$ é uma aplicação pura entre $\langle K, v\rangle$ e $\left\langle K^{\prime}, v^{\prime}\right\rangle$ extendendo $\phi$, pois $v(F)=v\left(\underset{n \in \mathbb{N}}{U} E_{n}\right)=\underset{n \in \mathbb{N}}{U}\left(v\left(E_{n}\right)\right)=\underset{n \in \mathbb{N}}{U} \widetilde{H}_{n}=v^{\prime}\left(F^{\prime}\right)$ é p $\underline{u}$ ro e enumerävel uma vez que $\tilde{H}_{n}$ são puros e enumerảveis. Finalmente seja $c \in F(a)$, então $c=\frac{f(a)}{g(a)} \operatorname{com} f, g \in F[X]$, $f=\sum_{i=0} a_{i} X^{i}, \underset{j=0}{g=b_{j} X^{j}}$, como $F=\underset{n \in \mathbb{N}}{U} E_{n}, a_{i} \in E_{n_{i}}, b_{j} \in E_{n_{j}}$, e como a sequencia dos $E_{n}$ e crescente existe $n \in \mathbb{N}$ tal que $a_{i}, b_{j} \in E_{n}$, e portanto $f, g \in E_{n}[X]$ donde $\operatorname{cEE}_{n}(a)$. Logo $v(c) \in v\left(E_{n}(a)\right)=H_{n} \subset \underset{n \in \mathbb{N}}{U} \tilde{H}_{n}=$ $=v(F)$. Isto e $v(F(a)) \subset v(F)$ e portanto $v(F(a))=v(F)$.

LEMA II.4 - Seja $\phi: E \rightarrow E$ ' uma aplicação pura entre $<K, v>$ e $\left\langle K^{\prime}, V^{\prime}\right\rangle$. Se $\langle K, V\rangle$ e $\left\langle K^{\prime}, V^{\prime}\right\rangle$ (além das condições impostas no início da seção) são w-pseudocompletos e satisfazem o Lema de Hensel, então existe uma aplicação pura $\Phi: \widetilde{\mathrm{E}}_{\mathrm{K}} \rightarrow \tilde{\mathrm{E}}_{\mathrm{K}}^{\prime}$ ' entre $\langle K, V\rangle$ e $\left\langle K^{\prime}, V^{\prime}\right\rangle$ extendendo $\phi\left(\tilde{E}_{K} \bar{e}\right.$ o fecho algébrico de E em K) .

\section{DEMONST RAÇÃO}

Seja $D$ o conjunto de todos os isomorfismos analiticos de finidos entre subcorpos de $\tilde{E}_{K}$ e $\widetilde{E}_{K}^{\prime}$ ' que extendem $\phi$.

$D \neq \phi$, pois $\phi \in D$; e dada uma cadeia em $D$ a reunião dos ele mentos da cadeia ê um elemento de $D$ e é o máximo da cadeia. Logo, pelo Lema de $\mathbb{Z}$ orn, existe $\Phi: F \rightarrow F^{\prime}$ elemento maximal $\operatorname{em} D$. 


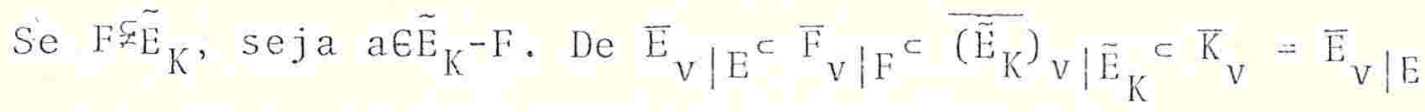
segue que $\overline{\mathrm{F}}_{v \mid F}=\left(\overline{\overrightarrow{\mathrm{E}}}_{K}\right)_{v \mid \overrightarrow{\mathrm{E}}_{K}} ;$ e de $v(E) \subset v(F) \subset v\left(\tilde{\mathrm{E}}_{K}\right) \subset \widetilde{v}(E)_{G}=v(E)$ segue que $v(E)=v(F)=v\left(\tilde{E}_{K}\right)$. Logo $\left\langle\tilde{E}_{K}, v\right| \tilde{E}_{K}>$ è uma extensão imediata de $\langle\mathrm{F}, V \mid F\rangle$ e como $v(F)$ é enumerảvel, segue pelo co rolário da proposição I5.1 que a $\tilde{E}_{K}-F$ é pseudolimite de algu ma sequência enumerável $\left\{a_{n}\right\}_{n<\omega}$ pseudoconvergente em $F$ e sem pseudolimites em F. Como a $\vec{e}$ algêbrico sobre $F, f(a)=0$ para algum $0 \neq f \in F[x]$ e portanto $f\left(a_{n}\right) \rightarrow f(a)=0$ o que mostra que $\left\{a_{n}\right\}_{n<\omega} \quad$ è algébrica em $F$.

Seja $q \in F[x]$ o polinômio minimal de $\left\{a_{n}\right\}_{n}<\omega \cdot\langle F, v \mid F\rangle \vec{e}$ fi nitamente ramificado pois $\langle K, v\rangle \quad 0 \vec{e} ; \mathrm{e}\langle K, v\rangle$ satisfaz o Lema de Hensel. Logo pela proposição I5.4 existe bEK pseudolimite de $\left\{a_{n}\right\}_{n<\omega}$ e tal que $q(b)=0$. Em particular b é algëbrico so bre $F$ e portanto tambëm sobre $E$, isto $\vec{e} b \tilde{E}_{K}$.

Por outro lado, é fäcil ver, que em F'[X] a sequência $\left\{\tilde{\phi}\left(a_{n}\right)\right\}_{n<\omega} \vec{e}$ pseudoconvergente, algébrica, sem pseudolimites e com polinômio minimal $q^{\prime}=\tilde{\phi} \circ q \in F^{\prime}[X]$; pois $\tilde{\phi} \vec{e}$ um isomorfismo analítico.

$<K^{\prime}, v^{\prime}>$ é $\omega$-pseudocompleto, logo existe a'EK' pseudolimite de $\left\{\tilde{\phi}\left(a_{n}\right)\right\}_{n<\omega}$ e portanto novamente pode-se aplicar a proposi ção I5.4 para concluir que existe b'€K' pseudolimite de $\left\{\tilde{\phi}\left(a_{n}\right)\right\}_{n<\omega}$ e tal que $q^{\prime}\left(b^{\prime}\right)=0$, donde $b^{\prime} \vec{e}$ algébrico sobre $F^{\prime}$ e portanto tambèm sobre E' e b' $\epsilon \tilde{E}^{\prime} K^{\prime}$ 
Por um argumento similar ao da parte (ii) do teorema 15.2 a ap1icação $\psi: F(b) \rightarrow F^{\prime}\left(b^{\prime}\right)$ definida por $\psi(f(b))=\tilde{\phi} \circ f\left(b^{\prime}\right)$ pa ra $f \in F x$, grfegrq $\vec{e}$ um isomorfismo analítico que extende $\tilde{\phi}$ e que pertence a $D$, contradizendo assim a maximalidade de $\bar{\phi}$ em $D$. Logo $F=\tilde{E}_{K}$ e analogamente (extendendo $\bar{\phi}^{-1}$ e depois invertendo) $F^{\prime}=\tilde{E}_{K}^{\prime}, . \operatorname{Logo} \Phi: \tilde{E}_{K} \rightarrow \tilde{E}_{K}^{\prime}$, . Finalmente como $\left(\overline{\widetilde{E}}_{K}\right)_{V} \mid \widetilde{E}_{K}$ $=\bar{K}_{v},\left.\left(\overline{\mathrm{E}}_{K^{\prime}}^{\prime}\right)_{v^{\prime}}\right|_{\mathrm{E}_{K^{\prime}}^{\prime}}=\overline{\mathrm{K}}_{v^{\prime}}^{\prime}$ e $v\left(\tilde{\mathrm{E}}_{\mathrm{K}}\right)=v(\mathrm{E})=v^{\prime}\left(\mathrm{E}^{\prime}\right)=v^{\prime}\left(\tilde{\mathrm{E}}_{\mathrm{K}^{\prime}}^{\prime}\right)$ s ão subgrupos puros e enumerâveis de $G$ e $\phi$ era aplicação pura, segue que $\Phi \vec{\phi}$ aplicação pura entre $\langle K, v\rangle$ e $\left\langle K^{\prime}, V^{\prime}\right\rangle$.

LEMA II.5 - Sejam $\langle K, v\rangle$ e $\left\langle K^{\prime}, V^{\prime}\right\rangle$ como no lema anterior e se ja $\phi: E \rightarrow E^{\prime}$ uma aplicaçäo pura entre $\langle K, V\rangle$ e $\left\langle K^{\prime}, V^{\prime}\right\rangle$ com $E$ algebricamente fechado em $K$. Se aEK-E é tal que $v(E(a))=v(E)$, então existe $\psi: E(a) \rightarrow E^{\prime}\left(a^{\prime}\right)$ aplicação pura entre $\langle K, v>\quad e$ $\left\langle K^{\prime}, V^{\prime}\right\rangle$ extendendo $\phi$.

DEMONSTRACSAO

De $\bar{E}_{v \mid E} \subset \overline{E(a)}{ }_{v \mid E}(a) c \bar{K}_{v}=\bar{E}_{v \mid E}$ segue que $\bar{E}_{v \mid E}=\overline{E(a)} v \mid E(a) ;$ e como $v(E(a))=v(E),\langle E(a), v \mid E(a)\rangle \vec{e}$ uma extensão imediata de $\langle E, v \mid E\rangle$. Pelo corolärio da proposição I5.I (pois v(E) ë enumerável) aEE(a)-E é pseudolimite de alguma sequência enumerável $\left\{a_{n}\right\}_{n<\omega}$ de $E$ pseudoconvergente e sem pseudolimites em E.

Se $\left\{a_{n}\right\}_{n<\omega}$ fosse algëbrica em E, com polinômio minimal qEE[X], então existiria bEK pseudolimite de $\left\{a_{n}\right\}_{n<\omega}$ e tal que 
$q(b)=0$. Como $\mathrm{E} \vec{e}$ algebricamente fechado em $K$, b pertenceria $\left.a \mathrm{E} e \mathrm{a}_{n}\right\}_{n<\omega}$ teria um pseudolimite em E. Logo $\left\{a_{n}\right\}_{n<\omega} \vec{e}$ trans cendente em E.

$\phi \vec{e}$ um isomorfismo analítico. $\operatorname{Logo}\left\{\phi\left(a_{n}\right)\right\}_{n<\omega} \vec{e}$ uma sequência pseudoconvergente transcendente em $\mathrm{E}^{\prime}$, e como $\left\langle\mathrm{K}^{\prime}, \mathrm{V}^{\prime}\right\rangle \overline{\mathrm{e}}$ $\omega$-pseudocompleto, existe a'EK' pseudolimite de $\left\{\phi\left(a_{n}\right)\right\}_{n<\omega}$.

Por um argumento similar ao da parte (ii) de teorema 15.1 a aplicação $\psi: E(a) \rightarrow E^{\prime}\left(a^{\prime}\right)$ definida por $\psi\left(\frac{f(a)}{g(a)}\right)=\frac{\phi o}{\phi \circ\left(a^{\prime}\right)} \quad f, g \in E[X]$ é um isomorfismo analítico extendendo $\phi$. Finalmente como $\overline{E(a)}{ }_{v}\left|E(a)=\bar{K}_{V}, \overline{E^{\prime}\left(a^{\prime}\right)} v^{\prime}\right| E^{\prime}\left(a^{\prime}\right)=\bar{K}_{v^{\prime}}$ e $v(E(a))=v(E)=v^{\prime}\left(E^{\prime}\right)=$ $=v^{\prime}\left(E^{\prime}\left(a^{\prime}\right)\right)$ é subgrupo puro e enumerävel de G e $\phi$ era aplicação pura, segue que $\psi$ é aplicação pura entre $\langle K, v\rangle$ e $\left\langle K^{\prime}, v^{\prime}\right\rangle$. PROPOSICGAO II.5 - Sejam $\langle K, V\rangle$ e $\left\langle K^{\prime}, V^{\prime}\right\rangle$ como nos lemas anteriores, $\phi: E \rightarrow E^{\prime}$ uma aplicação pura entre $\langle K, v\rangle$ e $\left\langle K^{\prime}, V^{\prime}\right\rangle$ e a EK. Então existe uma aplicação pura $\psi: F \rightarrow F^{\prime}$ entre $\langle K, v>$ e $\left\langle K^{\prime}, V^{\prime}\right\rangle$ extendendo $\phi$ e tal que a $\in F$.

\section{DEMONSTRAÇAO}

Nesta demonstração vamos intercalar as construções dadas pela proposição II.4 e pelo lema II.4 para extender $\phi$ a uma aplicação pura $\phi_{\omega}: \mathrm{E}_{\omega} \rightarrow \mathrm{E}_{\omega}^{\prime}$, onde $\mathrm{E} \overrightarrow{\mathrm{e}}$ algebricamente fechado em $\mathrm{K} \mathrm{e}$ $v\left(E_{\omega}(a)\right)=v\left(E_{\omega}\right)$. O lema anterior então nos darä a $\psi: E_{\omega}(a) \rightarrow$ $\rightarrow E_{\omega}^{\prime}\left(a^{\prime}\right)$. Para isto vamos fixar a seguinte notação:

Se $\phi: E \rightarrow E^{\prime}$ é uma aplicação pura entre $\langle K, v\rangle$ e $\left\langle K^{\prime}, v^{\prime}\right\rangle$ e 
a $\in K$, vamos indicar por $\hat{\phi}: \hat{\mathrm{E}} \rightarrow \widehat{E} \cdot$ a aplicação pura dada pela proposição II.4 que extende $\phi$ e tal que $v(\hat{E}(a))=v(\widehat{E})$; e vamos indicar por $\tilde{\phi}: \tilde{E} \rightarrow \widetilde{E}^{\prime}$ a aplicação pura dada pelo 1ema II.4 que extende $\phi$ a $\tilde{\mathrm{E}}=\tilde{\mathrm{E}}_{\mathrm{K}}$ e $\tilde{\mathrm{E}}^{\prime}=\tilde{\mathrm{E}}_{\mathrm{K}^{\prime}}^{\prime}$.

Como estas notações, seja $\phi_{n}: E_{n} \rightarrow E_{n}^{\prime}$ a seguinte sequência crescente de aplicações puras, construida indutivamente:

$$
\begin{aligned}
& \phi_{0}=\phi: E_{0}=E \rightarrow E_{0}^{\prime}=E^{\prime} \\
& \phi_{2 n+1}=\tilde{\Phi}_{2 n}: E_{2 n+1}=\tilde{E}_{2 n} \rightarrow E_{2 n+1}^{\prime}=\tilde{E}_{2 n}^{\prime} \\
& \phi_{2 n+2}=\widehat{\phi}_{2 n+1}: E_{2 n+2}=\widehat{E}_{2 n+1} \rightarrow E_{2 n+2}^{\prime}=\widehat{E}_{2 n+1}^{\prime} \\
& \text { e seja } \phi_{\omega}=\underset{n \in \mathbb{N}}{U} \phi_{n}: E_{\omega}=\underset{n \in \mathbb{N}}{U} E_{n} \rightarrow E_{\omega}^{\prime}=U_{n \in I N} E^{\prime} .
\end{aligned}
$$

Como cada $\phi_{n}$ é isomorfismo de corpos, segue que $\phi_{\omega}$ é isomorfismo de corpos.

$\left.\left.v\left(E_{\omega}\right)=v \underset{n \in \mathbb{N}}{U} E_{n}\right)=\underset{n \in \mathbb{N}}{U} v\left(E_{n}\right)=\underset{n \in \mathbb{N}}{U} v^{\prime}\left(E_{n}^{\prime}\right)=v^{\prime} \underset{n \in \mathbb{N}}{U} E_{n}^{\prime}\right)=v^{\prime}\left(E_{\omega}^{\prime}\right)$.

Se aEE ${ }_{\omega}$, então a $E_{n}$ para algum nEIN e portanto $v^{\prime}\left(\phi_{\omega}(a)\right)=$ $=v^{\prime}\left(\phi_{n}(a)\right)=v(a)$ o que mostra que $\phi_{\omega} \bar{e}$ isomorfismo analitico.

$$
\pi^{v\left(E_{\omega}\right)}=\pi^{n \in I N} v^{U\left(E_{n}\right)}=\underset{n \in \mathbb{N}}{U}\left(\pi^{v\left(E_{n}\right)}\right) \subset \underset{n \in \mathbb{N}}{U} E_{n}=E_{\omega} \text { e analogamente }
$$
$\pi, V^{\prime}(E \dot{\omega}) \subset E_{\omega}^{\prime}$.

Se $\alpha \in v\left(E_{\omega}\right)$, então $\alpha \in v\left(E_{n}\right)$ para algum $n \in \mathbb{N}$ e portanto $\phi_{\omega}\left(\pi^{\alpha}\right)=\phi_{n}\left(\pi^{\alpha}\right)=\pi^{\alpha}$ o que mostra que $\phi_{\omega} \bar{e}$ isomorfismo $x$-analí tico.

Finalmente como $v\left(E_{\omega}\right)=n \in \mathbb{H}_{\mathbb{N}} v\left(E_{n}\right) \vec{e}$ uma reunião enumerável 
de subgrupos puros e enumeráveis de $G$, então $v\left(E_{\omega}\right)=v^{\prime}\left(E_{\omega}^{\prime}\right)$ é um subgrupo puro e enumerável de G. E portanto $\phi_{\omega}$ é uma apli cação pura entre $\langle K, v\rangle$ e $\left\langle K^{\prime}, v^{\prime}\right\rangle$.

Seja $\alpha \in v\left(E_{\omega}(a)\right)$, então $\alpha=v\left(\frac{f(a)}{g(a)}\right) f, g \in E_{\omega}[X]$. Logo existe $n \in \mathbb{N}$ suficientemente grande e par tal que $f, g \in E_{n}[X]$, donde $\frac{f(a)}{g(a)} \in E_{n}(a)$ e $\alpha \in v\left(E_{n}(a)\right)=v\left(E_{n}\right) \subset v\left(E_{\omega}\right)$. E portanto $v\left(E_{\omega}(a)\right)=$ $=v\left(E_{\omega}\right)$.

Seja cEK algëbrico sobre $E_{\omega}$. Então $f(c)=0$ para algum 0ffEE ${ }_{\omega}[X]$ e portanto existe $n \in \mathbb{N}$ suficientemente grande e im par tal que $\mathrm{fEE}_{n}[\mathrm{X}]$, donde $c$ é algébrico sobre $E_{n}$ e portanto cEE ${ }_{n} \subset E_{\omega}$. Isto é $E_{\omega}=\left(\tilde{E}_{\omega}\right)_{K}$.

TEOREMA II. 2 (Ax e Kochen)

Sejam $\langle K, v\rangle$ e $\left\langle K^{\prime}, v^{\prime}\right\rangle$ dois corpos valorados, não ramifi cados, com o mesmo índice de ramificação (zero ou um), w-pseu docompletos, satisfazendo o Lema de Hensel e admitindo $x$-seções normalizadas. Se a cardinalidade de $K$ e $K^{\prime}$ e $\lambda_{1}$, $V(K)=V^{\prime}\left(K^{\prime}\right)=G$ e $\bar{K}_{V} \cong \bar{K}_{V^{\prime}}^{\prime}$, então existe um isomorfismo $x$-ana litico entre $\langle K, V\rangle$ e $\left\langle K^{\prime}, V^{\prime}\right\rangle$.

DEMONSTRAÇAO

Como $K$ e $K^{\prime}$ tem cardinalidade $K_{1}$ sejam $K=\left\{a_{\rho}: \rho<\omega_{1}\right\}$ e $K^{\prime}=\left\{a_{p}^{\prime}: \rho<\omega_{1}\right\}$, onde $\omega_{1}$ é o ordinal de $j_{1}$ (isto é: $\omega_{1} \bar{e}$ o menor ordinal de cardinalidade $\chi_{1}$ e foram escolhidas bijeções 
entre $\omega_{1}$ e $K$ e entre $\omega_{1}$ e $K^{\prime}$ e está sendo indicada por a $a_{\rho}$ $a_{p}^{\prime}$ a imagem destas bijeções no ponto $E^{\omega_{1}}$ ).

Agora serả construida, por indução transfinita, uma sequência crescente de aplicações puras $\phi_{\alpha}: E_{\alpha} \rightarrow E_{\alpha}^{\prime} \quad$ entre $\langle K, V\rangle$ e $\left\langle K^{\prime}, V^{\prime}\right\rangle$, para $\alpha<\omega_{1}$ :

$\phi_{0}: E_{0} \rightarrow E_{0}^{\prime}$ é a aplicação pura entre $\langle K, v\rangle$ e $\left\langle K^{\prime}, v^{\prime}\right\rangle$ dada pela proposição II.I ou pelo teorema II.1. conforme o ín dice de ramificação dos corpos seja zero ou um.

Construida $\phi_{\lambda+2 n}: E_{\lambda+2 n}{ }^{+E_{\lambda+2 n}^{\prime}}$, onde $\lambda<\omega_{1}$ e um ordinal limite e $n \in \mathbb{N}$; se $E_{\lambda+2 n}=K$, então $\phi_{\lambda+2 n+1}=\phi_{\lambda+2 n}$. Senão seja $\mu=\min \left\{\rho<\omega_{1}: a_{\rho} E E_{\lambda+2 n}\right\}, \phi_{\lambda+2 n+1}: E_{\lambda+2 n+1} \rightarrow E_{\lambda+2 n+1}^{\prime}$ será a aplicação pura entre $\langle K, v\rangle$ e $\left\langle K^{\prime}, v^{\prime}\right\rangle$ dada pela proposição anterior que extende $\phi_{\lambda+2 n}$ e tal que $a_{\mu} \in E E_{\lambda+2 n+1}$.

Construida $\phi_{\lambda+2 n+1}: E_{\lambda+2 n+1}+E_{\lambda+2 n+1}^{\prime}$, onde $\lambda<\omega_{1} \vec{e}$ un ordi nal limite e $n \in \mathbb{N}$; se $E_{\lambda+2 n+1}^{\prime}=K^{\prime}$, então $\phi_{\lambda+2 n+2}=\phi_{\lambda+2 n+1}$. Se não seja $\mu=\min \left\{\rho<\omega_{1}: a_{\rho}^{\prime} \notin E_{\lambda+2 n+1}^{\prime}\right\}$ e $\psi: F^{\prime} \rightarrow F$ a aplicação pu ra entre $\langle K, v\rangle$ e $\left\langle K^{\prime}, v^{\prime}\right\rangle$ que extende $\phi_{\lambda+2 n+1}^{-1}$ e tal que $a_{\mu}^{\prime} \in F^{\prime}$. A aplicação $\phi_{\lambda+2 n+2}$ será então $\psi^{-1}$.

Construidas $\phi_{\alpha}$ para $\alpha<\beta$, onde $\beta<\omega_{1} \vec{e}$ um ordinal limite, $\phi_{\beta}=\underset{\alpha<\beta}{U} \phi_{\alpha}: E_{\beta}=\underset{\alpha<\beta}{U} E_{\alpha} \rightarrow E_{\beta}^{\prime}=\underset{\alpha<\beta}{U} E_{\alpha}^{\prime}$.

$$
v\left(E_{\beta}\right)=\underset{\alpha<\beta}{U} v\left(E_{\alpha}\right)=\underset{\alpha<\beta}{U} v^{\prime}\left(E_{\alpha}^{\prime}\right)=v^{\prime}\left(E_{\beta}^{\prime}\right) \text {, cada } v\left(E_{\alpha}\right) \dot{e} \quad \text { um }
$$

subgrupo puro e enumerável de $G$ e $\beta$ é enumerâvel pois $\beta<\omega_{1}$. 
Logo $v\left(E_{\beta}\right)=V^{\prime}\left(E_{\beta}^{\prime}\right)$ é uma reunião enumerâvel de subgrupos puros e enumeráveis de $G$ e portanto é um subgrupo puro e enume rável de G. E por uma argumentação similar a da proposição anterior, onde se mostrava que $\phi_{\omega}$ era uma aplicação pura, se gue que $\phi_{\beta}$ é uma apłicação pura entre $\langle K, v\rangle$ e $\left\langle K^{\prime}, v^{\prime}\right\rangle$ que ex tende $\phi_{\alpha}$ para todo $\alpha<\beta$.

E estä assim terminada a construção da sequência $\phi_{\alpha}: E_{\alpha} \rightarrow E_{\alpha}^{\prime}$, para $\alpha<\omega_{1}$. Seja agora:

$\psi=\underset{\alpha<\omega_{1}}{U} \phi_{\alpha}: F=\underset{\alpha<\omega_{1}}{U} E_{\alpha}+F^{\prime}=\underset{\alpha<\omega_{1}{ }^{\prime}}{U} E^{\prime}$

Pela argumentação citada acima, $\psi$ é uma aplicação x-analitica cutre $\langle K, v\rangle$ e $\left\langle K^{\prime}, V^{\prime}\right\rangle$ (neste caso não se pode garantir que $\psi$ é uma aplicação pura, pois năo se pode garantir que $V(F)=\underset{\alpha<\omega{ }_{1}}{U} V\left(E_{\alpha}\right)=\underset{\alpha<\omega_{1}}{U} V^{\prime}\left(E_{\alpha}^{\prime}\right)=V^{\prime}\left(F^{\prime}\right)$ e enumerävel); e mostran do que $F=K$ e $F^{\prime}=K^{\prime}$ estarä demonstrado o teorema.

A demonstração de $F=K$ será feita mostrando-se, por indu ção transfinita, que a $\in F$ para todo $0<\omega_{1}$ :

Supondo que $\rho<\omega_{1}$ ( $\rho$ pode ser ordinal limite ou ordinal sucessor) e que a $\in F$ para todo $\sigma<p$, então para cada $\sigma<p$ exis te $\alpha_{\sigma}<\omega_{1}$ tal que $a_{\sigma} \in E_{\alpha_{\sigma}}$. Seja $\beta=U_{\sigma<p} \alpha_{\sigma}$, $\beta$ é reunião enumerävel de ordinais enumeráveis e portanto $\beta$ é um ordinal enumeráve1, isto $\overline{\mathrm{e}} \beta<\omega_{1}$ e portanto existe $\mathrm{E}_{\beta}>\mathrm{E}_{\alpha_{\sigma}}$ para todo $\sigma<\rho$. Pela construção da sequência $\phi_{\alpha}$, segue então que a $\in_{\rho} E_{\beta+2}$, e portanto que a $E_{\rho}$. 
Assim $F=K$ e analogamente $F^{\prime}=K^{\prime}$ e $\psi: K \rightarrow K^{\prime}$ é o isomorfismo $x$-analítico procurado. 
Conforme a notação apresentada no prefácio, um corpo $K$ se diz $C_{i}(d)$ quando toda forma (polinômio homogêneo) de gra u d em $n>d^{i}$ variäveis com coeficientes em $k$, admitir uma ra iz não trivial em $K$. O corpo $K$ se diz $C_{i}$ se for $C_{i}$ (d) para todo inteiro positivo d.

Artin conjecturou que os corpos $Q_{p}$ seriam $C_{2}$. Nesta seção apresentamos um contraexemplo devido à G. Terjanian, de uma forma de grau 4 em 18 variäveis que não admite raizes não triviais em $Q_{2}$, mostrando assim que $Q_{2}$ não $\vec{e} C_{2}(4)$. Nas pröximas seçöes deste capitulo introduziremos os rudimentos da Teoria dos Modelos necessários para o resultado de Ax e Kochen sobre a conjectura de Artin e concluiremos o capítu10 com este resultado; a saber: que para cada inteiro positivo d o conjunto $F(d)$ de todos os numeros primos p para os quais $Q_{p}$ não é $C_{2}(d)$ e finito. (Veja no prefácio deste tra- 
balho para maiores informações sobre a conjectura de Artin e o resultado de Ax e Kochen).

O contraexemplo de Terjanian: Seja

$$
\begin{aligned}
f\left(x_{1}, x_{2}, x_{3}\right)= & x_{1}^{4}+x_{2}^{4}+x_{3}^{4}-x_{1}^{2} x_{2}^{2}-x_{1}^{2} x_{3}^{2}-x_{2}^{2} x_{3}^{2}-x_{1}^{2} x_{2} x_{3}- \\
& -x_{1} x_{2}^{2} x_{3}-x_{1} x_{2} x_{3}^{2}, \text { e sejam } x_{1}, x_{2}, x_{3} \text { tres }
\end{aligned}
$$

nümeros inteiros. Se pelo menos um dos tres nümeros for impar, então $f\left(x_{1}, x_{2}, x_{3}\right)=1(\bmod 4) ; \quad$ e se todos forem pares, então $f\left(x_{1}, x_{2}, x_{3}\right)=0(\bmod 4)$.

Seja $g\left(x_{1}, \ldots, x_{9}\right)=f\left(x_{1}, x_{2}, x_{3}\right)+f\left(x_{4}, x_{5}, x_{6}\right)+$ $+f\left(x_{7}, x_{8}, x_{9}\right)$. Então $g\left(x_{1}, \ldots, x_{9}\right)=0(\bmod 4) \quad\left(\right.$ para $x_{1}, \ldots$ $x_{9}$ inteiros) se e só se todos os $x_{i}$ são pares.

Finalmente seja $h\left(x_{1}, \ldots, x_{18}\right)=g\left(x_{1}, \ldots, x_{9}\right)+$ $4 g\left(x_{10}, \ldots, x_{18}\right)$, e sejam $x_{1}, \ldots, x_{18}$ inteiros. Se $h\left(x_{1}, \ldots, x_{18}\right)=0(\bmod 16)$, então $g\left(x_{1}, \ldots, x_{9}\right)=0(\bmod 4)$, donde $x_{i}=2 y_{i} y_{i} \in \mathbb{Z}$ para $i=1, \ldots, 9$ e $g\left(x_{1}, \ldots, x_{9}\right)=$ $g\left(2 y_{1}, \ldots, 2 y_{9}\right)=2^{4} g\left(y_{1}, \ldots, y_{9}\right)=0(\bmod 16)$. Dai segue que $4 g\left(x_{10}, \ldots, x_{18}\right)=0(\bmod 16)$ e portanto $g\left(x_{10}, \ldots, x_{18}\right)=0(\bmod 4)$ donde $x_{i}$ é par para todo $i=1, \ldots, 18$.

Da proposição I4.1 sabemos que todo elemento reQ 2 se escreve de forma unica como $r={ }_{i}^{\infty} \sum_{\ell}^{\infty} c_{i}(r) \cdot 2^{i} \quad$ com $c_{i}(r)=0$ ou 1 e $c_{\ell}(r) \neq 0$, onde $\ell=v_{2}(r)$. Os elementos de $R_{<Q_{2}, v_{2}>}$ poderão então ser descritos como $a={ }_{i=0}^{\infty} c_{i}(a) \cdot 2^{i}$ (onde ago ra $c_{0}(a)$ pode $\operatorname{ser}=0$ ). 
A aplicação $\wedge$ que a cada $a \in R_{<Q_{2}}, v_{2}>$ associa $\hat{a}=$ $\left\{{ }_{i=0}^{n-1} c_{i}(a) \cdot 2^{i}\right\}_{\bmod } 2^{n}=$ a classe de equivalência de ${ }_{i=0}^{n} c_{i}(a) \cdot 2^{i}$ em $z_{2} n$ è um epimorfismo de anëis, pois dados $a, b \in R_{<Q_{2}}, v_{2}>$ temos: $a={ }_{i=0}^{n_{i}}{ }_{0}^{1} c_{i}(a) \cdot 2^{i}+2^{n} r_{1}$, $b={ }_{i=0}^{n} \sum_{0}^{1} c_{i}(b) \cdot 2^{i}+2^{n} r_{2}$ e $a+b={ }_{i}^{n} \sum_{0}^{1} c_{i}(a+b) \cdot 2^{i}+2^{n} r_{3}$, com $r_{1}, r_{2}, r_{3} \in R_{<Q_{2}}, v_{2}>$, donde $z={ }_{i=0}^{n} \sum_{0}^{1} c_{i}$ (a) $2^{i}+{ }_{i=0}^{n} \sum_{i}^{1} c_{i}$ (b) $2^{i}-{ }_{i=0}^{n} \sum_{i}^{1} c_{i}(a+b) 2^{i}=2^{n_{r}}, \quad$ com $r \in R_{<Q_{2}, v_{2}>}$ e portanto $v_{2}(z)=n$. Como $z \in \mathbb{Z}$ segue que

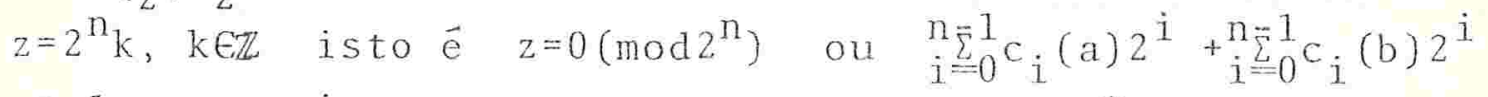
$={ }_{i}^{n} \sum_{0}^{1} c_{i}(a+b) 2^{i}\left(\bmod 2^{n}\right)$ e portanto $\hat{a}+\hat{b}=\widehat{a+b}$ em $\mathbb{Z}_{2} n$. Analogamente $\hat{a} \cdot \hat{b}=\widehat{a} \cdot \hat{b}$. $(0$ núcleo deste epimorfismo $\overrightarrow{\mathrm{c}}$ $\left(I_{<Q_{2}, v_{2}>}\right)^{n}$, de modo que temos: $R_{<Q_{2}, v_{2}>} /\left(I_{<Q_{2}, v_{2}>}\right)^{n} \simeq \mathbb{Z}_{2}{ }^{n}$ resultado este que generaliza $R_{\left\langle Q_{2}, v_{2}>\right.} / I_{\left.<Q_{2}, v_{2}\right\rangle} \simeq \mathbb{Z}_{2} \cdot$ )

Suponhamos agora que $\left(r_{1}, \ldots, r_{18}\right) \in_{2}$ ë uma raiz não trivial de $h$ em $Q_{2}$. Seja $j$ um indice tal que $v\left(r_{j}\right) \leq v\left(r_{i}\right)$ para todo $i=1, \ldots, 18$. E claro que $r_{j} \neq 0$ (senão a 18 -üpla seria trivial) e portanto $\left(a_{1}, \ldots, a_{18}\right)$, onde $a_{i}=r_{i} / r_{j}$ é uma raiz não trivial de $h$ que pertence a $\left(R_{<Q_{2}, v_{2}>}\right)^{18}$, uma vez que $v\left(a_{i}\right)=v\left(r_{i}\right)-v\left(r_{j}\right) \geq 0$ para todo $i=1, \ldots, 18$; com $a_{j}=1$.

Aplicando $\wedge$, com $n=3$, à igualdade $h\left(a_{1}, \ldots, a_{18}\right)=0$ teremos que: $h\left(\hat{a}_{1}, \ldots, a_{18}\right)=\{0\}_{\text {mod16 }}$ e portanto $a_{i}=\left\{x_{i}\right\} \bmod 16^{\circ}$, com $x_{i}$ par, contra o fato que $\hat{a}_{j}=\{1\} \bmod 16^{\circ}$ 


\section{$\S 1$. UMA INTRODUÇÃO $\bar{A}$ TEORIA DOS MODELOS}

A teoria dos modelos é oramo da lógica matemática que estuda as relaçóes entre teorias e seus modelos.

Uma teoria, neste contexto, é qualquer conjunto de sen tenças de uma linguagem formal dada; e um modelo de uma te oria é qualquer estrutura para a linguagem que satisfaz to das as sentenças da teoria.

Nesta seção apresentaremos definições formais para todos os conceitos grifados e daremos exemplos de descrição de teorias matemáticas familiares (grupos, corpos, etc.) neste formalismo.

DEFINICAO IIII.1 - Uma einguagem de primeira ordem L è um conjunto de tres espêcies de objetos: simbolos de predicado, simbolos de função e simbolos de constante, tais que:

a cada símbolo de predicado p de L estâ associado um nümero ineiro positivo $n$, e costumamos designar isto dizen do que $\mathrm{p}$ é um símbolo de predicado de $n$ lugares ou que $p$ un símbolo de predicârio n-ário.

a cada símbolo de função $f$ de $L$ estả associado um nüme ro inteiro positivo $\mathrm{m}$, e costumamos designar isto dizendo que $f$ é um símbolo de função de m lugares ou que $f$ è um símbolo de função m-äria.

EXEMPLOS : Conforme o assunto que se pretende estudar, escolhem-se os símbolos para a linguagem. Por exemplo:

1. uma linguagem adequada para o estudo de conjuntos 
ordenados è $L=\{\leq\}$, onde é um sïmbolo de predicado binärio.

2. uma linguagem adequada para o estudo de grupos ê $L=\{*, e\}$, onde * è um sîmbolo de função binäria e e é um símbolo de constante. Uma outra possibilidade seria:

$L=\{*, f, e\}$, onde $f \vec{e}$ um símbolo de função unäria (que cor responderá, num grupo, à função que a cada elemento associa o seu inverso) e uma outra possibilidade seria simplesmente $L=\left\{{ }^{*}\right\}$. Em todas elas nös poderemos enunciar axiomas para a teoria dos grupos mas conforme a linguagem utilizada poderemos escolher axiomas diferentes (veja em exemplos de te orias).

3. $\mathrm{L}=\left\{\leq,^{*}, e\right\}$ è uma linguagem para grupos ordenados.

4. $\mathrm{L}=\{+, \cdot, 0,1\}$ è uma 1 inguagem para anẻis com unidade ou para corpos.

DEFINICÁO IIII.2 - Seja $L=\left\{p_{i}: i \in I\right\} U\left\{f_{j}: j \in J\right\} U\left\{c_{k}: k \in K\right\} \quad$ uma linguagem de $1 \mathrm{a}$. ordem. Uma estrutura para L Cou uma L-estrutura) $\vec{e}$ um par ordenado $A=\langle|A|, \bar{A}\rangle$, onde $|A| \vec{e}$ um conjunto não vazio, chamado universo da estrutura $A$ e $\bar{A} \vec{e}$ uma função, chamada interpretação da estrutura $A$, definida em L e tal que:

Se p é símbolo de predicado n-ârio de $L$, então $\bar{A}(p)$ é um subconjunto de $|A|^{n}$ (isto $\vec{e} \bar{A}(p)$ è uma relação n-äria do universo da estrutura).

Se $f$ é um símbolo de função m-äria de $L$, então $\bar{A}(p)$ é 
uma função definida em $|A|^{m}$ com valores em $|A|$.

Se c è um símbolo de constante de $L$, então $\bar{A}(c)$ é um $\underline{e}$ lemento de $|A|$.

Costuma-se designar $\bar{A}(p), \bar{A}(f), \bar{A}(c)$ por $p^{A}, f^{A}, c^{A} \operatorname{respec}$ tivamente; dizer que $p^{A} \bar{e}$ a interpretação do símbolo de predicado $p$ na estrutura $A, f^{A} \vec{e}$ a interpretação de $f$ em $A$ e $c^{A} \vec{e}$ a interpretação de $c$ em $A$; e escrever, por abuso de notação, $A=\left\langle|A|,\left(p_{i}^{A}\right)_{i \in I},\left(f_{j}^{A}\right)_{j \in J},\left(C_{k}^{A}\right)_{k \in K}\right\rangle$ para uma $L-$ estrutura. Se $L$ é finita $L=\left\{_{0}, \ldots, p_{i}, f_{0}, \ldots, f_{j}, c_{0}, \ldots, c_{k}\right\}$ então escreve-se simplesmente $A=\left\langle|A|, p_{0}^{A}, \ldots, p_{i}^{A}, f_{0}^{A}, \ldots\right.$, $\mathrm{f}_{j}^{A}, c_{0}^{A}, \ldots, c_{k}^{A}>$ para uma L-estrutura.

\section{EXEMPLOS :}

1. para a linguagem $L=\{\leq\}$ (do 1 : exemplo de 1inguagens) uma L-estrutura $\vec{e}$ qualquer par $A=\langle A, R\rangle$, onde $A \neq \phi$ $\vec{e}$ o universo de $A$ e $\operatorname{RcA}^{2}$ é a relação binária $\leq^{A}$ do universo de $A$ que corresponde ao símbolo de predicado binärio s de L. Observe que por enquanto não hä necessidade de $R$ satisfazer qualquer outra condição, alêm de $R_{c} A^{2}$ (e $A \neq \varnothing$ ) para que $\langle A, R\rangle$ seja uma estrutura para a linguagem dos con juntos ordenados. Após as definições de satisfatibilidade, veremos que para que $\langle A, R\rangle$ seja um modelo da teoria dos conjuntos ordenados ề que $R$ terâ que ser uma relação de or dem de A.

2. uma estrutura para a linguagem $L=\{*$,e $\}$ da teoria 
dos grupos $\vec{e}$ uma trinca $G=\left\langle G,{ }^{G}, e^{G}\right\rangle$, onde $G \neq \phi$, ${ }^{*}: G \times G \rightarrow G$ e $e^{G} \in G$. Às vezes, quando não houver perigo de confusão, escreveremos simplesmente $\langle\mathrm{G}$, *, e $\rangle$ usando a mesma notação para os símbolos da linguagem e para suas inter pretações na estrutura.

3. as quadruplas $\left\langle\mathbb{N},\left\{<m, n>\mathbb{N}^{2}: m \mid n\right\},\left\{<<m, n>, k>\mathbb{N}^{2} \times \mathbb{N}\right.\right.$ : $m \cdot n=k\}, 1>$ e $\left\langle\mathbb{Z},\left\{\langle p, q\rangle \in \mathbb{Z}^{2}: p \leq q\right\},\left\{\langle\langle p, q\rangle, p+q\rangle \in \mathbb{Z}^{2} \times \mathbb{Z}\right\}, 0\right\rangle \quad$ são ambas exemplos de estruturas para a linguagem $L=\{\leq, *, e\}$. Com as definições adequadas a $2 \mathrm{a}$. estrutura serä um modelo da teoria de grupos ordenados, enquanto que a la. não o se rả por não satisfazer as sentenças daquela teoria.

4. $\left\langle\mathbb{Z},\left\{\langle\langle p, q\rangle, p+q\rangle \in \mathbb{Z}^{2} \times \mathbb{Z}\right\},\left\{\left\langle\left\langle p, q>, p \cdot q>\in \mathbb{Z}^{2} \times \mathbb{Z}\right\}, 0,1>\right.\right.\right.$ e $<\mathrm{Q},\left\{<\left\langle\mathrm{r}, \mathrm{s}>, \mathrm{r}+\mathrm{s}>\in \mathrm{Q}^{2} \times \mathrm{Q}\right\},\left\{\left\langle<\mathrm{r}, \mathrm{s}>, \mathrm{r} \cdot \mathrm{s}>\in \mathrm{Q}^{2} \times \mathrm{Q}\right\}, 0,1>\right.\right.$ são estrutu ras para a linguagem $\mathrm{L}=\{+, \cdot, 0,1\} \cdot \mathrm{A} 2 \mathrm{a} \cdot$ serä um modelo da teoria dos corpos, enquanto que a primeira será apenas um modelo da teoria de anêis com unidade.

Duas estruturas para uma mesma linguagem dizem-se estruturas similares ou de mesmo tipo. Para estruturas simila res definem-se os conceitos de homomorfismo, monomorfismo, etc. que unificam as definições correspondentes de várias teorias matemäticas. Veremos aqui, a título de exemplo des te procedimento, a definição de isomorfismo.

DEFINICRAO IIII.3 - Sejam A, B duas estruturas similares (e seja L a 1 inguagem comum a ambas). Uma aplicação $h:|A| \rightarrow|B|$ 
entre os universos das estruturas se diz um isomorfismo en tre A e B se h é bijetora e:

1. para todo símbolo de predicado n-ário p de $L$ e para todos $a_{1}, \ldots, a_{n} \in|A|,<_{1}, \ldots, a_{n}>\in p^{A}$ se e só se $\left\langle\mathrm{h}\left(\mathrm{a}_{1}\right), \ldots, \mathrm{h}\left(\mathrm{a}_{\mathrm{n}}\right)>\in \mathrm{p}^{\mathrm{B}}\right.$.

2. para todo símbolo de função m-äria $f$ de $L$ e para todos $a_{1}, \ldots, a_{m} \in|A|, h\left(f^{A}\left(a_{1}, \ldots, a_{m}\right)\right)=f^{B}\left(h\left(a_{1}\right), \ldots, h\left(a_{m}\right)\right)$. 3. para todo símbolo de constante $c$ de $L, h\left(c^{A}\right)=c^{B}$.

Os elementos de uma linguagem (símbolos de predicado, de função e de constante) são também chamados de símbolos näo lógicos ou parametros da linguagem. Eles desempenham o papel de letras do alfabeto de una lingua que se usam na construção das palavras e frases da língua (termos e fórmu las neste contexto). Além dêstes símbolos (que são particu lares para cada linguagem) precisaremos de mais alguns que são comuns a todas as linguagens e são chamados de símboIos lögicos:

1. parênteses: ), (;

2. variāveis: um conjunto infinito e enumerảvel $v=\left\{v_{0}, v_{1}, \ldots, v_{n}, \ldots\right\}$

3. conectivos proposicionais: ᄀ (não), $\wedge$ (e);

4. quantificador universa1: $\forall$ (para todo);

5. Simbolo de igualdade: $=$

Justapondo simbolos de uma linguagem constroem-se va- 
rias expressões, por exemplo: $\left.v_{\leq}\right)\left(v_{1}=+(\right.$ ou $\left(\left(0+v_{2}\right) \cdot\left(1+v_{1}\right)\right)+1$ ou $\quad v_{v_{1}}\left(0+v_{2}=1+v_{1}\right)$. E c1aro que nem to das as expressões nos interessam. Vamos então discriminar entre todas as expressões possĩveis de uma linguagem dois tipos importantes: os termos que são expressões, como a do 2: exemplo, que descrevem objetos construidos a partir das variäveis, dos sỉmbolos de constante e de aplicaçöes sucessivas dos símbolos funcionais, que são como os nomes e pronomes de uma língua; e as förmulas que são expressões, como a do 3: exemplo, que descrevem asserções entre termos.

DEFINICCAO IIII.4 - Seja L uma linguagem de la. ordem e seja C o conjunto dos símbolos de constante de L. Vamos construir indutivamente uma sequência de conjuntos $\mathrm{T}_{\mathrm{k}}, \mathrm{k} \in \mathbb{N}$, como segue:

$$
\begin{aligned}
& \mathrm{T}_{0}=\mathrm{V} u \mathrm{C}=\text { conjunto de todas as variâveis e de todos } \\
& \text { os símbolos de constante de } L \text {. } \\
& \mathrm{T}_{\mathrm{k}+1}=\mathrm{T}_{\mathrm{k}} \cup\left\{\mathrm{f}\left(\mathrm{t}_{1} \ldots \mathrm{t}_{\mathrm{m}}\right): \text { f } \vec{e}\right. \text { um símbolo de função } \\
& \left.\mathrm{m} \text {-äria de } \mathrm{L} \text { e } t_{1}, \ldots, \mathrm{t}_{\mathrm{m}} \in \mathrm{T}_{\mathrm{k}}\right\} \text {. }
\end{aligned}
$$

Entäo definimos o conjunto dos termos de L como sendo:

$$
\operatorname{Ter}(\mathrm{L})=\underset{k \in \mathbb{N}}{u} \mathrm{~T}_{k} .
$$

DEFINICAO III1.5 - Seja L uma Iinguagem de 1a. ordem e seja Ter(L) o conjunto dos termos de L. Vamos construir indutiva mente uma sequência de conjuntos $F_{k}$, $k \in \mathbb{N}$, como segue: 


$$
\mathrm{F}_{0}=\left\{\left(t_{1}=t_{2}\right): t_{1}, t_{2} \in \operatorname{Ter}(L)\right\} \cup\left\{p\left(t_{1} \ldots t_{n}\right): p e \vec{e}\right. \text { um }
$$
símbolo de predicado $n$-ärio de $L$ e $\left.t_{1}, \ldots, t_{n} \in \operatorname{Ter}(L)\right\}$.

$$
\begin{gathered}
F_{k+1}=F_{k} \cup\left\{\neg A: A \in F_{k}\right\} \cup\left\{(A \wedge B): A, B \in F_{k}\right\} \\
u\left\{\forall v A: \quad v \in V, A \in F_{k}\right\} .
\end{gathered}
$$

o conjunto $\mathrm{F}_{0}$ se diz o conjunto das fórmulas atômicas de $\mathrm{L}$ e o conjunto $\operatorname{For}(L)=\underset{k \in \mathbb{N}}{u} F_{k}$ se diz o conjunto das fórmulas de $L$.

Introduziremos algumas abreviações ususais, para tornar as expressões mais comunicativas, através dos símbolos:

$v(o u),+$ (implica), $\leftrightarrow$ (equivale) e $\exists$ (existe). Assim escreveremos:

$$
\begin{aligned}
& (A \vee B) \text { para } \neg(\neg A \wedge \neg B), \\
& (A \rightarrow B) \text { para } \neg(A \wedge \neg B), \\
& (A \leftrightarrow B) \text { para }((A \rightarrow B) \wedge(B \rightarrow A)), \\
& \exists \vee A \text { para } \neg \forall \vee \neg A .
\end{aligned}
$$

Omitiremos alguns dos parênteses quando isto não causar confusão. Para símbolos predicativos e símbolos funcionais binários u escreveremos frequentemente $\left(t_{1} u t_{2}\right)$ ao invés de $u\left(t_{1} t_{2}\right)$. Assim por exemplo na linguagem $L=\{\leq,+, \ldots, 0,1\}$ escreveremos $\left(v_{1}+1\right) \cdot v_{3} \leq v_{1}+\left(1 \cdot v_{3}\right)$ ao invés de $\leq\left(\cdot\left(+\left(v_{1} 1\right) v_{3}\right)+\left(v_{1}\left(1 \cdot v_{3}\right)\right)\right)$.

o proximo passo agora $\vec{e}$ o de definir quando que uma es- 
trutura para uma linguagem satisfaz uma förmula daquela lin guagem .

De imediato observamos (intuitivamente) que enquanto certas fórmulas, como por exemplo $0<1$ ou $\exists v_{1} v v_{2}\left(v_{1} \leq v_{2}\right)$, ou serão verdadeiras ou falsas numa dada estrutura; existem outras, como por exemplo $v_{2} \leq 1$ ou $\quad v_{2}\left(v_{1} \leq v_{2}\right)$, cuja satisfatibilidade numa dada estrutura dependerá, por assim dizer, do valor que for dado a algumas das variáveis que nelas ocorrem.

Começaremos então definindo quando que uma estrutura satisfaz uma förmula para uma dada substituição das variäveis por elementos do universo da estrutura.

DEFINICAO III1.6 - Sejam A uma L-estrutura e $\mathrm{s}: \mathrm{V} \rightarrow|\mathrm{A}|$ uma função definida no conjunto das variäveis com valores no universo da estrutura. Vamos definir o que significa dizer que uma fórmula $A$ da linguagem $L \vec{e}$ satisfeita pela substituiçãos na estrutura $A$ (ou que A satisfaz A com's), e denotar isto por $A \vDash A[s]$.

Inicialmente vamos extender $s$ para $\bar{s}: \operatorname{Ter}(L) \rightarrow|A| \operatorname{def} \underline{i}$ nida no conjunto dos termos da linguagem $L$. Informalmente isto corresponde a efetuar as operações que comparecem nos termos usando para as variáveis o valor dado pela s. Formalmente a definição $\vec{e}$ indutiva para seguir a definição in dutiva dos termos. 
1. para cada variäve1 $\mathrm{v}, \overline{\mathrm{s}}(\mathrm{v})=\mathrm{s}(\mathrm{v})$,

2. para cada símbolo de constante $c, \bar{s}(c)=c^{A}$,

3. se $t_{1}, \ldots, t_{m}$ são termos e $f$ um símbolo de função m-ária então $\bar{s}\left(f\left(t_{1} \ldots t_{m}\right)=f^{A}\left(\bar{s}_{\left(t_{1}\right)}, \ldots, \bar{s}\left(t_{m}\right)\right)\right.$.

Observe que os dois primeiros itens desta definição definem $\bar{s}$ em $\mathrm{T}_{0}$, enquanto que o terceiro define $\overline{\mathrm{s}} \mathrm{em} \mathrm{T}_{\mathrm{k}+1}$ a partir da definição de $\overline{\mathrm{s}}$ em $\mathrm{T}_{k}$; de modo que esta definição è de fato uma definição indutiva. No futuro procederemos como acima (inclusive para fórmulas) sem contudo chamarmos a atenção para o processo de indução envolvido em ca da caso.

Passemos agora à definição de $A F A[S]$ que será também indutiva:

1. Se $t_{1}$ e $t_{2}$ são termos, então $A F t_{1}=t_{2}[s]$ se e só se $\bar{s}\left(t_{1}\right)=\bar{s}\left(t_{2}\right)$,

2. Se $t_{1}, \ldots, t_{n}$ são termos e p um símbolo de predicado n-ärio, então $A \mid p\left(t_{1} \ldots t_{n}\right)[s]$ se e só se

$$
\left\langle\bar{s}\left(t_{1}\right), \ldots, \bar{s}\left(t_{n}\right)\right\rangle \in p^{A} \text {, }
$$

3. Se $A$ é $\neg B$, então $A F A[s]$ se e sö se $A$ não satis faz $B$ com $s$ (em símbolos $A \not B[s]$ ),

4. Se $A$ e $B \wedge C$, então $A F A[S]$ se e só se $A \mid B[s]$ e $A \vDash C[s]$,

5. Se $A$ é $\forall V B$, então $A \neq A[s]$ se e só se para todo $a \in|A|$, 
$A \vDash B[s(v \mid a)]$, onde estamos denotando por $s(v \mid a)$ a função definida em V com valores em $|A|$ que coincide com s para as variáveis distintas de $v$ e em v toma o valor a, isto é: $s(v \mid a)(x)=s(x)$ se $x \neq v$ e $s(v \mid a)(v)=a$. (Logo $A \mid \forall_{V} B[s]$ se e só se A satisfaz $B$ com todas as substitui ções que coincidem com $s$ fora de $v$ ).

OBSERVAÇÃO - O conjunto das variäveis $V$ jâ foi definido co mo sendo o conjunto de todos os simbolos $v_{i}$ para $i \in N$, de modo que quando dizemos que "v ë una variävel" estamos que rendo dizer que $v$ é $v_{i}$ para algum iEN (e não que v é algum simbolo novo).

Da definição anterior segue facilmente que:

1. $A \vDash B \vee C[s]$ se e sô se $A \vDash B[s]$ ou $A \vDash C[S]$

2. $A \models B \rightarrow C[s]$ se e sö se $A \not \neq B[s]$ ou $A \neq C[s]$

(isto é se $A \vDash B[S]$, então $A \vDash C[S]$ )

3. $A \vDash B \leftrightarrow C[s]$ se e só se A satisfaz ambas as förmulas com $s$ ou não satisfaz nenhuma das duas com s.

4. $A \models \exists v B[s]$ se e só se existe a $|A|$ tal que $A \models B[s(v \mid a)]$ (isto é A satisfaz B com alguma substituição que coinci de com $s$ fora de $v$ ).

Uma substituição s dả um valor a cada variảvel v de $V$, mas para uma förmula nunca precisaremos de todos estes va- 
lores, uma vez que só pode ocorrer um nümero finito de variảveis em cada fórmula. Além disto tambëm para as variáveis da fómula que estão quantificadas não interessara valor da substituição. Esta ideia dará origem a uma proposição assim que se defina formalmente o que são as variâveis livres de uma fórmula (isto é as variảveis que não es tão no escopo de algum quantificador $\forall$ e $\equiv$ ).

Para isto precisamos definir inicialmente quais as variảveis que ocorrem num termo, mas deixaremos esta definição (que se faz por indução de forma natural) para o leitor. Indicaremos por var(t) o conjunto das variäveis que ocorrem num termo $t$, e iremos agora definir o conjunto v1(A) das variáveis livres de uma förmula A.

DEFINIÇAO IIII. 7 -

1. Se $t_{1}$ e $t_{2}$ são termos, então $v 1\left(t_{1}=t_{2}\right)=\operatorname{var}\left(t_{1}\right) u$ $v \operatorname{var}\left(t_{2}\right)$,

2. Se $t_{1}, \ldots, t_{n}$ são termos e p um simbolo de predicado n-ärio, então $\operatorname{vi}\left(p\left(t_{1} \ldots t_{n}\right)\right)=\operatorname{var}\left(t_{1}\right) u \ldots \operatorname{vvar}\left(t_{n}\right)$,

3. $v 1(\neg A)=v 1(A)$,

4. $v 1(A \wedge B)=\operatorname{vi}(A) \cup v 1(B)$,

5. $v 1\left(\forall_{V A}\right)=v_{1}(A)-\{v\}$.

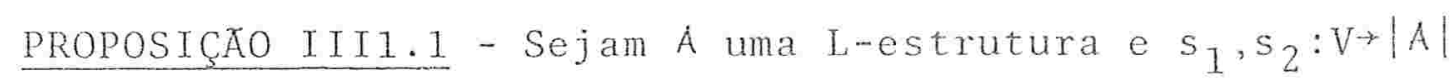

(i) Se $t$ é um termo de $L$ tal que $s_{1}$ e $s_{2}$ coincidem nas variảveis de $t$, então $\bar{s}_{1}(t)=\bar{s}_{2}(t)$ 
(ii) Se A é uma förmula de $L$ tal que $s_{1}$ e $s_{2}$ coincidem nas variảveis livres de $A$, então $A F A\left[S_{1}\right]$ se e sö se $A F A\left[s_{2}\right]$.

DEMONSTRACĀO

(i) por indução sobre termos:

1. Se $t$ é uma variävel $v$, então $v a r(t)=\{v\}$ e portanto $\bar{s}_{1}(t)=\bar{s}_{1}(v)=s_{1}(v)=s_{2}(v)=\bar{s}_{2}(v)=\bar{s}_{2}(t)$ pela hipötese sob re $s_{1}$ e $s_{2}$.

2. Se $t$ è uma constante $c$, entäo $\bar{s}_{1}(c)=c^{A}=\bar{s}_{2}(c)$.

3. Se $t$ é $f\left(t_{1} \ldots t_{m}\right)$, então $s_{1}$ e $s_{2}$ coincidem nas variáveis de cada um dos $t_{i} e$, por hipötese de indução $\bar{s}_{1}\left(t_{i}\right)=\bar{s}_{2}\left(t_{i}\right)$ para $i=1, \ldots, m$. Logo $\begin{aligned} \bar{s}_{1}(t) & =\bar{s}_{1}\left(f\left(t_{1} \ldots t_{m}\right)\right)=f^{A}\left(\bar{s}_{1}\left(t_{1}\right), \ldots, \bar{s}_{1}\left(t_{m}\right)\right)= \\ & =f^{A}\left(\bar{s}_{2}\left(t_{1}\right), \ldots, \bar{s}_{2}\left(t_{m}\right)\right)=\bar{s}_{2}\left(f\left(t_{1} \ldots t_{m}\right)\right)=\bar{s}_{2}(t) .\end{aligned}$

(ii) por indução sobre fôrmulas:

1. Se $A$ é $t_{1}=t_{2}$, então $s_{1}$ e $s_{2}$ coincidem em var $\left(t_{1}\right)$ uvar $\left(t_{2}\right)$, e portanto, pela parte $(i), \bar{s}_{1}\left(t_{1}\right)=\bar{s}_{2}\left(t_{1}\right)$ e $\bar{s}_{1}\left(t_{2}\right)=$ $\bar{s}_{2}\left(t_{2}\right)$, donde $\bar{s}_{1}\left(t_{1}\right)=\bar{s}_{1}\left(t_{2}\right)$ se e só se $\bar{s}_{2}\left(t_{1}\right)=\bar{s}_{2}\left(t_{2}\right)$ ou $A \vDash A\left[s_{1}\right]$ se e so se $A \vDash A\left[s_{2}\right]$.

2. Analogamente se A $\vec{e} p\left(t_{1} \ldots t_{n}\right)$.

3. Se $A \vec{e} \neg B$, então $s_{1}$ e $s_{2}$ coincidem em vI(B) e, por hipó tese de indução, $A \mid B\left[s_{1}\right]$ se e só se $A \neq B\left[s_{2}\right]$, e portanto $A \not F\left[s_{1}\right]$ se e só se $A \not B B\left[s_{2}\right]$, ou $A F A\left[s_{1}\right]$ se e só se $A=A\left[s_{2}\right]$.

4. Analogamente se $A \vec{e} B \wedge C$. 
5. Se $A$ é $\forall v B$, então $s_{1} \in s_{2}$ coincidem em $v 1(B)-\{v\}$, e portanto para todo aE $|A|, s_{1}(v \mid a)$ e $s_{2}(v \mid a)$ coincidem em v1(B). Logo, por hipótese de indução, para todo a $|A|$, $A \vDash B\left[s_{1}(v \mid a)\right]$ se e só se $A \vDash B\left[s_{2}(v \mid a)\right]$, ou $A \vDash A\left[s_{1}\right]$ se e só se $A \neq A\left[s_{2}\right]$.

Uma fórmula da linguagem L que não possui variäveis 1 i $\underline{\text { }}$ res serả dita uma sentença de L. Vamos indicar por Sent(L) - conjunto de todas as sentenças de L, assim $\operatorname{Sent}(L)=\{A \in \operatorname{For}(L): \operatorname{vl}(A)=\phi\}$. A proposição anterior aplicada à sentenças nos fornece imediatamente o seguinte: COROLARRIO - Sejam A uma L-estrutura e A una sentença de L. Então para quaisquer duas substituições $\mathrm{s}_{1}, \mathrm{~s}_{2}: \mathrm{V} \rightarrow|\mathrm{A}|$ tem-se: $A \vDash A\left[s_{1}\right]$ se e só se $A \neq A\left[s_{2}\right]$.

DEFINICAO IIII.8 - Sejam A uma L-estrutura e A uma förmula de L. Diremos que $A \vec{e}$ verdadeira ou vălida em $A$ ou que A satisfaz ou $\vec{e}$ modelo de $A$, e denotar este fato por $A \vDash A$, quando ocorrer que A satisfaz A com toda substituição S:V A. Se A for vâlida em todas as L-estruturas, então diremos que A é universalmente välida ou simplesmente valida e denotaremos isto pork $A$.

Observe que se A é uma sentença de L que não $\vec{e}$ vầida em A, então rA é vâlida em A ou seja: se A é sentença, então ou A é vảlida ou $\rightarrow$ A $\vec{e}$ vảilida em $A$ (e apenas uma das du 
as, pois $|A| \neq \phi)$; enquanto que se $A$ é uma förmula qualquer pode ocorrer que nem A seja välida em A nem $\neg$ A seja välida em A (por exemplo $v_{1}=v_{2}$ numa estrutura cujo universo tenha pe10 menos dois elementos).

Seja ScSent(L). Se todas as sentenças de $S$ forem välidas em A diremos que $A \vec{e}$ modelo de $S$ e denotaremos isto por $A \mid=S$. Se $S$ admite algum modelo dizemos que $S$ é satisfatîvel ou (semanticamente) consistente, caso conträrio que ele é contraditōrio ou (semanticamente) inconsistente. Se uma sentença A é válida em todos os modelos de S dizemos que A é conseqliencia (semantica) de $S$ ou que $S$ implica (semantica mente) A e denotamos isto por $S \vDash A$. No caso de $S=\{B\}$ escreveremos $\mathrm{B} F \mathrm{~A}$ ao inves de $\{\mathrm{B}\} \models \mathrm{A}$. Se $\phi \models \mathrm{A}$, então $\models \mathrm{A}$ (isto è A è universalmente vâlida).

Alëm destas definições "semânticas" existem outras "sin täticas", que thes são equivalentes. Nos livros de teoria de modelos, após as demonstrações de equivalencia, unificam -se as definições, suprimindo-se delas as palavras "semânt co" e "sintätico". Aqui não veremos este desenvolvimento, mas mesmo assim suprimiremos a palavra "semântico" das defi nições.

Se toda consequência de $S$ pertence a $S$, dizemos que $S$ é uma teoria. O conjunto de todas as sentenças välidas em A: $\operatorname{Th}(A)=\{A \in \operatorname{Sent}(L): A \vDash A\}$ e o conjunto de todas as con sequências de $S: \operatorname{Cnsq}(S)=\{A \in \operatorname{Sent}(L): S \vDash A\}$ são exem 
plos de teorias.

Se uma teoria $\Sigma$ é consistente (isto ê $\sum_{\ddagger} \operatorname{Sent}(L)$ ) e não existe nenhuma teoria consistente $\tau$ contendo propriamente (isto é não existe teoria $\tau$ tal que $\sum_{z} \tau \varsubsetneqq \operatorname{Sent}(L)$ ), então dizemos que $\sum$ é completa. Não é difícil de ver que as teorias Th( $A)$ são completas. E imediato também que se $A$ é modelo de $S$, então $A$ è modelo de Cnsq(S). Costuma-se dizer que $S$ é um conjunto de axiomas para a teoria $\Sigma$ quando Cnsq $(S)=\Sigma$, de modo que podemos dizer, como era de se espe-rar, que se uma estrutura satisfaz todos os axiomas de uma teoria, então ela satisfaz também todas as consequências deles.

PROPOSICAO III1.2 - Sejam A e B duas L-estruturas, $h:|A| \rightarrow|B|$ um isomorfismo entre $A$ e $B$ e $s: V \rightarrow|A|$. Então:

(i) $\mathrm{h}(\overline{\mathrm{s}}(\mathrm{t}))=\overline{\mathrm{h} o \mathrm{~s}}(\mathrm{t})$ para todo termo $t \in \operatorname{Ter}(\mathrm{L})$

(ii) $A \neq A[S]$ se e sô se $B \neq A[h \circ s]$, para toda $A \in$ For (L)

(iii) $A \vDash A$ se e sô se $B \vDash A$, paratoda $A \in \operatorname{For}(L)$. DEMONSTRAÇAO

(i) se faz por indução sobre os termos como na proposi ção IIII.I

(ii) será demonstrado por indução sobre fórmulas que para toda $s: V \rightarrow|A|$ e toda fôrmula $A$ For $(L), A \vDash A[s]$ se é só se $B=A[h \circ s]$. 
Quando A é atômica isto segue imediatamente do 1 i.tem, do fato de h ser um isomorfismo e da definição de satisfati bilidade .

Quando $A \vec{e} T B$ ou $A \vec{e}$ BA $C$ o resultado segue tambëm ime diatamente da definição de satisfatibilidade.

Se $A \vec{e} \forall v B$, então $A \vDash \forall v B[s]$ se e sö se para todo a $|A|$ $A \vDash B[s(v \mid a)]$ que por hipōtese de indução equivale a: para todo $a \in|A|, B \vDash B[h \circ(s(v \mid a))]$ que, como $h \circ(s(v \mid a))=$ $(h \circ s)(v \mid h(a))$, equivale a: para todo $a \in|A|$, $B \vDash B[(h \circ s)(v \mid h(a))]$ que, por ser h bijetora, equivale a: para todo $b \in|B|, \quad B F B[(h \circ s)(v \mid b)]$ ou seja $B \vDash \forall V B[h \circ s]$.

(iii) ê consequência imediata de (ii) e do fato de $h$ ser bijetora.

Duas estruturas similares que satisfazem as mesmas förmulas dizem-se elementarmente equivalentes. Isto significa que são indistinglíveis por sentenças de Ia. oxdem. Estrutu ras isomorfas são elementarmente equivalentes, mas é possível que estruturas não isomorfas também sejam elementarmente equivalentes. Mais adiante apresentaremos um exemplo.

\section{EXEMPLOS DE TEORIAS:}

1. Na linguagem $L=\{\leq\}$ podemos enunciar axiomas para a teoria de ordem parcial, que são: 
$\mathrm{O}_{1} \cdot \quad \forall v_{0}\left(\mathrm{v}_{0} \leq \mathrm{v}_{0}\right)$

$\mathrm{O}_{2} \cdot \quad v_{v_{0}} v_{1}\left(v_{0} \leq v_{1} \wedge v_{1} \leq v_{0}+v_{0}=v_{1}\right)$ (estamos escrevendo $\forall v_{0} v_{1}$ ao invës de $\forall v_{0} \forall v_{1}$ )

o. $\quad \forall v_{0} v_{1} v_{2}\left(v_{0} \leq v_{1} \wedge v_{1} \leq v_{2} \rightarrow v_{0} \leq v_{2}\right)$

De acordo com a definição de satisfatibilidade, uma es trutura $A=\left\langle|A|, R c|A|^{2}\right\rangle$ para esta 1 inguagem, será um modelo para a teoria de ordem parcial se e só se:

para todo $a \in|A|, \quad<a, a>E R$

para todos $a, b \in|A|$, se $\langle a, b>\in R$ e $\langle b, a>\in R$, então $\mathrm{a}=\mathrm{b}$.

para todos $a, b, c \in|A|$, se $<a, b>\in R$ e $<b, c>\in R$, então $<\mathrm{a}, \mathrm{c}>\in R$.

ou seja $R$ é de fato uma relação de ordem parcial de $|A|$.

Colocando mais um axioma:

$0_{4} \cdot \quad \forall x y\left(x \leq y^{\vee} y \leq x\right) \quad$ (onde $x$ e $y$ são duas variâveis quais. quer)

teremos a teoria de ordem total.

Com mais os axiomas:

$\mathrm{O}_{5} \cdot \quad \forall x y(x \leq y \wedge x \neq y \rightarrow \exists z(x \leq z \wedge x \neq z \wedge z \leq y \wedge y \neq z))$

$\mathrm{O}_{6} \cdot \quad \exists x y(x \neq y)$

teremos a teoria de ordem densa. Os modelos desta teoria são conjuntos totalmente ordenados com mais de um elemento e tais que entre dois elementos quaisquer sempre existe um terceiro.

Podemos tambëm enunciar axiomas, nesta linguagem, para 
a teoria de ordem densa sem extremos, acrescentando:

$0_{7} \cdot \forall x \exists y(y \leq x \wedge y \neq x)$

$0_{8} \cdot \forall x \exists y(x \leq y \wedge y \neq x)$

E um teorema de Cantor que dois modelos enumeráveis quais quer desta teoria são sempre isomorfos. Se todos os modelos de cardinalidade $\alpha$ de uma teoria são isomorfos, ela se diz a-categōrica. A teoria de ordem densa sem extremos $\vec{e}$ então $t_{0}$-categórica. E um teorema da teoria dos modelos (teste de Vaught) que se uma teoria de uma linguagem, que tem no máximo um número enumerável de sîmbolos não 1ỏgi-cos, só admite modelos infinitos e é a-categórica para al gum a infinito, então ela é completa. A teoria de ordem densa sem extremos é portanto completa (uma vez que é ime diato de ver que os modelos desta teoria são infinitos). Se uma teoria $\vec{e}$ completa, então dois modelos quaisquer da teoria são elementarmente equivalentes (ê suficiente olhar com cuidado as duas definiçōes). Em particular os racionais com a ordem usual e os reais com a ordem usual são modelos da teoria de ordem densa sem extremos e portanto são elementarmente equivalentes e obviamente eles não são isomorfos.

Não é possível porên enunciar nesta linguagem axiomas para uma teoria de boa ordem. Isto ê: (demonstra-se que) não existe um conjunto de sentenças (finito ou infinito) desta linguagem tal que uma estrutura $A=\left\langle|A|, R c|A|^{2}\right\rangle$ 
è modelo deste conjunto se e só se $R$ é uma relação de boa ordem de $|A|$. E isto (informalmente) se deve ao fato que na definição de boa ordem quantifica-se sobre subconjun tos de $|A|$, enquanto que os quantificadores de uma senten ça quando testada na estrutura percorrem apenas os elemen tos do universo $|A|$.

2. Na linguagem $L=\{*, e\}$ podem-se enunciar axiomas para a teoria dos grupos:

$G_{1} \cdot \quad \forall x y z((x * y) * z=x *(y * z))$

$\mathrm{G}_{2} \cdot \quad \forall \mathrm{x}\left(\mathrm{x} * e=\mathrm{x} \wedge e^{*} \mathrm{x}=\mathrm{x}\right)$

$\mathrm{G}_{3} \cdot \quad \forall x \exists y\left(x^{*} y=e \wedge y^{*} x=e\right)$

enquanto que na Iinguagem $L=\{*, f, e\}$ pode-se enunciar, ao invés de $\mathrm{G}_{3}$, :

$\mathrm{G}_{3}^{-} \cdot \quad \forall \mathrm{x}(\mathrm{x} * \mathrm{f}(\mathrm{x})=e \wedge \mathrm{f}(\mathrm{x}) * \mathrm{x}=e)$

Nesta formulação todos os axiomas estão escritos usan cdo-se apenas o conectivo $\wedge$ e o quantificador $\forall$, enquanto que na 1a. formulação foi necessārio usar-se o quantificador $\exists$ (substituindo $\exists x A$ por $\neg \forall x \neg A$ ocorreria então o conectivo ᄀ; e de fato não se pode naquela linguagem enunciar axiomas para a teoria dos grupos usando apenas $\wedge$ e $\forall$ ). Existem na teoria dos modelos alguns teoremas que relacionam o tipo de axiomas de uma teoria com a preservação dos modelos daquela teoria (por extensões, homomorfismos, etc., veja as seções 3.2 e 5.2 de $[\mathrm{C} . \mathrm{K}]$ ). Por exemplo, se 
$A=\left\langle|A|,{ }^{A}, f^{A}, e^{A}\right\rangle$ e um modelo da teoria dos grupos e $B=\left\langle|B|,{ }^{*} B, f^{B}, e^{B}\right\rangle$ é uma subestrura de $A$ (isto $\vec{e}|B| c|A|$, ${ }^{B} B$ é a restrição de $*^{A}$ a $|B|^{2}, f^{B}$ é a restrição de $C^{A}$ a $|B|$ e $e^{B}=e^{A}$ ), então $B$ tambëm è um modelo da teoria dos grupos.

3. E făcil agora enunciar-se axiomas na linguagem $L=\left\{\leq,{ }^{*}, e\right\}$ para a teoria dos grupos ordenados, não se pode contudo enunciar axiomas para a teoria dos grupos ordenados arquimedianos. (observação.: tanto neste exemplo quanto nos outros a impossibilidade de enunciar-se um con ceito em linguagem de $1 \mathrm{a}$. ordem è um fato que demonstrase na teoria dos modelos, porém a partir de resultados ma is fortes do que estes que estamos vendo nesta introdução Veja por exemplo em [C.K] o teorema da compacidade 1.3 .22 e seus corolários na seção 2.1).

4. Escrevendo, de acordo com o formalismo imposto nas linguagens de $1 \mathrm{a}$. ordem, os axiomas usuais de corpo, teremos axiomas na linguagem. $\mathrm{L}=\{+, \ldots, 0,1\}$ para a teoria dos corpos .

Dado um primo $\mathrm{p}$, acrescentando-se o axioma: $\mathrm{p} \cdot 1=$ $1+(1+(\ldots(1+1) \ldots))=0$, onde o termo 1 ocorre p vezes, tere mos axiomas para a teoria de corpos de característica p.

Se ao invés disto colocarmos, para cada primo $p$, a negação deste axioma, teremos a teoria de corpos de característica zero. Este $\vec{e}$ o primeiro exemplo que vemos de teo- 
ria infinitamente axiomatizäve1. A teoria dos corpos de ca racterística diferente de zero (porëm arbitrária) é outro exemplo de conceito que não pode ser enunciado em lingua gem de 1a. ordem (veja Corolärio 2.1.10 de [C.K]).

Um outro exemplo de teoria infinitamente axiomatizävel $\vec{e}$ dos corpos algebricamente fechados: Aos axiomas de corpo acrescenta-se para cada inteiro positivo n o seguinte axio ma: $\quad \forall x_{0} \ldots x_{n} y\left(x_{0} \cdot y^{n}+\ldots+x_{0}=0\right)$

(dado o número n explicitamente pode-se escrever esta fốrmula por extenso, sem o uso dos pontos, de acordo com as regras de formação de fórmulas).

Nesta seção apresentaremos um método de construção de estruturas a partir de uma familia de estruturas similares dada. Este método originou-se com os trabalhos de skolem em 1930, mas só foi largamente utilizado, após os traba 1hos de Los em 1955. Para um tratamento mais completo veja [C.K] Caps. 4, 5, 6 .

DEFINICAO III2.1 - Seja I um conjunto não vazio e seja D um conjunto cujos elementos são subconjuntos de I, is to é: $D \subset P(I)$. Diremos que:

1. D satisfaz a propriedade da interseção finita se: $x_{1}, \ldots, x_{n} \in D$ implica $x_{1} \cap \ldots \cap x_{n} \neq \phi$. 
2. D é um filtro sobre I se:

(i) $\quad I \in D$

(ii) $X, Y \in D$ implica $X \cap Y \in D$

(iii) $X \in D$ e $X \subset Y \quad I$ implicam $Y \in D$.

3. D è um ultrafiltro sobre I se:

(i) $\mathrm{D} \overrightarrow{\mathrm{e}}$ um filtro sobre I

(ii) para todo $X \subset I, X \in D$ se e söse $I-X \notin D$.

Um filtro sobre I serä dito proprio se não for igual a todo $P(I)$. E imediato de ver que se D è um filtro sobre I, são equivalentes:

(i) D é um filtro pröprio

(ii) $\varnothing \notin D$

(iii) D satisfaz a prorpiedade da interseção finita.

\section{EXEMPLOS:}

1. Dado $Y \quad I$, seja $\langle Y\rangle=\{X \subset I: Y \subset X\} .\langle Y\rangle \vec{e} u m$ filtro sobre I chamado filtro principal gerado por Y. $\langle Y\rangle$ será um ultrafiltro se e sö se $Y$ for unitärio. Neste caso escreveremos $\langle y\rangle$ para $\langle\{y\}\rangle$.

2. O conjunto $F=\{X \subset I: I-X \vec{e}$ finito $\} \vec{e}$ um filtro sobre I conhecido como o biltro de Fréchet de I, Ele serä pröprio se e só se I é infinito.

3. Se em I está definida uma topologia então o conjunto $V(x)$ das vizinhanças de um ponto $x \in I$ é um filtro sobre $I$, 
que será um ultrafiltro se e só se $\{x\}$ è um aberto de $I$ e neste caso $V(x)=\langle x\rangle$ : o ultrafiltro principal gerado por $\{x\}$.

4. E fácil de ver que interseção qualquer de filtros é flltro, de modo que dado $E \subset P(I)$ o conjunto $[E]=$ interseção de todos os filtros que contem E (e esta interseção é possí vel pois existe pelo menos um filtro que contem E: o filtro imprórpio P(I) )é um filtro sobre I chamado filtro gerado por E. O filtro $\langle Y\rangle \vec{e}$ um caso particular deste quando $E=\{Y\}$.

PROPOSICÃO III2.1 - Seja $E \subset P(I)$ e seja [E] ofiltro gerado por E: Então:

(i) $[E]=\left\{X \subset I\right.$ : $X=I$ ou existe $n \in N$ e $Z_{1}, \ldots, Z_{n} \in E$ tais que $\left.Z_{1} \cap \ldots n Z_{n} \in X\right\}$

(ii) [E] é un filtro pröprio se e sö se E satisfaz a propriedade da interseção finita.

DEMONSTRAÇAO

(i) Seja D o conjunto dos $X \subset I$ tais que $X=I$ ou existe $n \in N$ e $z_{1}, \ldots z_{n} \in E$ tais que $z_{1} \cap \ldots n z_{n} \in X$. Então:

1. $I \in D$

2. Se $x$, YGD, então $X \supset Z_{1} n \ldots n Z_{n}$ e $Y \supset Z_{1}^{\prime} n \ldots n Z_{m}^{\prime}$ de modo que $X \cap Y \supset Z_{1} \cap \ldots n Z_{n} \cap Z_{1}^{\prime} \cap \ldots n Z_{m}^{\prime}$ e portanto $X \cap Y \in D$ (o caso em que $X=I$ ou $Y=I$ é trivial).

3. Se $X \in D$ e $X \subset Y \subset I$, então $Z_{1} n \ldots n Z_{n} \subset X \subset Y$ de modo que $Y \in D$ (idem).

Logo D $\vec{e}$ um filtro sobre $I$ e como EcD segue que [E]CD. 
Por outro lado seja XED e sejam $z_{1}, \ldots, z_{n} \in E$ tais que $z_{1} \cap \ldots \cap z_{n} \subset X$. Como $[E] \vec{e}$ um filtro que contem $E$ segue que $Z_{1}, \ldots, Z_{n} \in[E]$ e pela condição (ii) sobre filtros segue que $Z_{1} \cap \ldots \cap Z_{n} E[E]$ e pela condição (iii) sobre filtros que $X \in[E]$. Logo $D \subset[E]$ e portanto $D=[E]$.

(ii) segue do item (i) desta proposição.

PROPOSICAOO III2.2 -

(i) D è um ultrafiltro sobre I se e sö se D ê um filtro próprio e maximal sobre I

(ii) Todo filtro pröprio sobre I pode ser extendido a um ultrafiltro sobre I.

DEMONSTRACÃO

(i) Seja D um ultrafiltro sobre I. Então $\phi \notin D$ pois $I-\phi=I \in D$. Logo D é próprio. Seja $D^{\prime} \geq D$ um filtro sobre I. En tão existe XED' tal que XÆD. Como D ë ultrafiltro I-XED e como DeD' segue que I-XED'. XED' e I-XED' implicam que $X(I-X)=\varnothing E D^{\prime}$ pois $D^{\prime} \vec{e}$ filtro e portanto $D^{\prime}=P(I), \quad$ o que mostrou que D $\vec{e}$ filtro pröprio maximal.

Reciprocamente seja D um filtro pröprio e maximal sobre $I$ e seja $X \subset I$. E claro que não se pode ter XED e I-XED senão $\phi=X \cap(I-X) \in D$ e $D$ não seria pröprio. Logo se XED, então I-X $\notin D$.

Por outro lado seja $I-X \notin D$, e seja $E=D u\{X\} . E$ satisfaz a propriedade da interseção finita, pois se $Y_{1}, Y_{2} \in E$, 
então $Y_{1}, Y_{2} \in D$ ou $Y_{1}^{n Y_{2}}=Y \cap X$ com YED. No primeiro ca so $\mathrm{Y}_{1} \cap \mathrm{Y}_{2} \neq \varnothing$ pois $\mathrm{D}$ é pröprio e no segundo caso $\mathrm{Y} \cap \mathrm{X} \neq \phi$ senão $Y \subset I-X$ e portanto $I-X \in D$ contra a suposição. Logo E é um filtro próprio e como d é maximal segue que $D=[E]$ e portanto $X \in D$. Isto ë se $I-X \notin D$, então $X \in D$; que junto com a primeira implicação nos dá: XeD se e só se I-X $\notin D$, e portanto que D è ultrafiltro.

(ii) Seja D um filtro próprio sobre I e seja C o conjun to de todos os filtros pröprios sobre I que contem D. Então $C \neq \varnothing$ pois $D \in C$, e se $\left\{\mathrm{F}_{\lambda}: \lambda \in L\right\}$ é uma cadeia não vazia de $C$ (isto é um subconjunto de C totalmente ordenado por inclu são), então $F=\left\{F_{\lambda}: \lambda \in L\right\}$ é um elemento de $C$ que majora $\left\{F_{\lambda}: \lambda \in L\right\}$, pois:

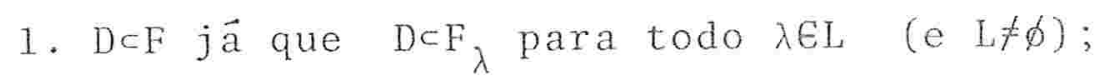

2. $\phi \notin F$ pois $\phi \notin F$ para todo $\lambda \in L$;

3. IEF já que $\operatorname{IEF}_{\lambda}$ para todo $\lambda \in L$ (e L $\left.\neq \phi \phi\right)$;

4. Se X,YEF, então XEF $\lambda_{1} \quad \mathrm{YEF}_{\lambda_{2}} \mathrm{e} \operatorname{como}\left\{\mathrm{F}_{\lambda}: \lambda \in L\right\}$ é uma ca deia $F_{\lambda_{1}} \subset F_{\lambda_{2}}$ ou $F_{\lambda_{2}} \subset F_{\lambda_{1}}$, donde $X, Y \in F_{\lambda}$ para algum $\lambda \in L$ e portanto $X \cap Y \in F_{\lambda}$ donde $X \cap Y \in F$;

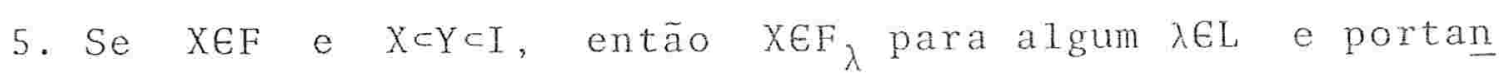
to $\mathrm{YEF}_{\lambda}$ donde $\mathrm{Y} \in \mathrm{F}$.

Pode-se aplicar então o Lema de Zorn ao conjunto $C$ para obter um elemento MEC maximal em C. M e um filtro pröprio e maximal sobre I, pois se F fosse um filtro próprio conten do $M$, então $F \supset M \supset D$ e portanto $F$ pertenceria a $C$ e pela ma- 
ximalidade de $M$ em $C$ seguiria que $F=M$. Pelo primeiro item desta proposição segue então que $M \vec{e}$ um ultrafiltro sobre I que contem D.

COROLARIO - Se I é infinito, então existem ultrafiltros não principais sobre I.

\section{DEMONSTRACAO}

Seja $F=\{X \in I: I-X$ ë finito $\}$ ofiltro de Frëchet sob re I. Como I $\vec{e}$ infinito, $F \vec{e}$ um filtro proprio e pela propo sição anterior existe D ultrafiltro sobre I que extende F. Se $X \in D$, então $X \vec{e}$ infinito senão $I-X$ tambëm pertenceria a D (por pertencer a F). Logo D é näo principal.

Seja D um filtro sobre um conjunto não vazio I e seja $\left\{A_{i}\right.$ : iEI $\}$ uma família de conjuntos indexados pelos elementos de $I$. O produto cartesiano $\prod_{i \in I}^{I} A_{i} \vec{e}$ o conjunto de todas as funções a definidas em $I$ e tais que a(i) $\in A_{i}$ para todo

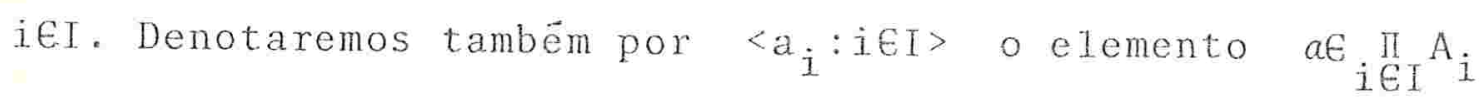
tal. que $a(i)=a_{i}$ para todo $i \in I$.

Vamos definir em $\prod_{i \in I} A_{i}$ uma relaçăo $a={ }_{D}^{b}$ (que se $1 e$ a é igual a b módulo D) por:

$$
\begin{aligned}
& a={ }_{D} b \quad \text { se e sö se }\{i \in I: a(i)=b(i)\} \in D . \\
& \text { E claro que } a={ }_{D} a \text { e que } a={ }_{D} b \text { implica } b={ }_{D} a \text {. }
\end{aligned}
$$

Tambëm se $a={ }_{D} b$ e $b={ }_{D} c$, então $\{i \in I: a(i)=b(i)\} \in D$ e $\{i \in I: b(i)=c(i)\} \in$ D. Como: 
$\{i \in I: a(i)=c(i)\} \supset\{i \in I: a(i)=b(i)\} n\{i \in I: b(i)=c(i)\} e$ D é um filtro segue que $\{i \in I: a(i)=c(i)\} \in D$ e portanto $a={ }_{D} c \cdot$ Logo ${ }_{D}$ é uma relação de equivalencia em $\underset{i \in I}{\operatorname{II}} A_{i}$ : Vamos denotar por $a_{D}$ ou por $\left\langle a_{i}: i \in I\right\rangle_{D}$ a classe de equivalencia de um elemento $a=<a_{i}: i \in I>\epsilon \underset{i \in I}{\Pi} A_{i}$.

Assim $a_{D}=\left\{b \in \underset{i \in I}{\prod_{i}} A_{i}: a{ }_{D} b\right\}$ e vamos denotar por $\prod_{i \in I} A_{i} / D$ o conjunto de todas as classes de equivalencia de $\prod_{i \in I} A_{i}$. As $\operatorname{sim} \prod_{i \in I} A_{i} / D=a_{D}: a \in \underset{i \in I}{\pi} A_{i}$. O conjunto $\prod_{i \in I} A_{i} / D$ será chamado o produto reduzido dos $A_{i}$ módulo $D$ e se D for um ultrafiI tro ele será chamado o ultraproduto dos $A_{i}$ módulo $D$.

DEFINIÇÃO III2.2 - Sejam L uma Iinguagem de la. ordem, $\left\{A_{i}: i \in I\right\}$ uma família de L-estruturas indexadas pelos ele mentos de um conjunto não vazio I e D un filtro sobre I. Va mos definir uma estrutura $A=\prod_{i \in I} A_{i} / D$ que será chamada - produto reduzido das estruturas $A_{i}$ módulo D:

1. 0 universo $|A|$ da estrutura $A$ serä o produto reduzido $\prod_{i \in I}^{\pi}\left|A_{i}\right| / D$ dos universos $\left|A_{i}\right|$ das estruturas $A_{i}$. Observe que como cada $\left|A_{i}\right| \neq \emptyset$ (por definição de estrutura) segue pelo Axioma da Escolha que $\prod_{i \in I}\left|A_{i}\right| \neq \phi$ e portanto $|A|=\underset{i \in I}{I}\left|A_{i}\right| / D \neq \phi$.

2. Se pé um símbolo predicativo n-ärio de L, então: $\mathrm{p}^{\mathrm{A}}=\left\{\left\langle a_{D}^{1}, \ldots, a_{D}^{\mathrm{n}}\right\rangle \in|A|:\left\{i \in I:\left\langle a^{1}(i), \ldots, a^{\mathrm{n}}(\mathrm{i})>\in \mathrm{p}^{\mathrm{A}}\right\} \in \mathrm{D}\right\}\right.$. 
Se $\quad a_{D}^{1}=b_{D}^{l}, \ldots, a_{D}^{n}=b_{D}^{n}$, entäo:

$\left\{i \in I:<b^{x}(i), \ldots, b^{n}(i)>\in p^{A} i\right\},\left\{i \in I:<a^{g}(i), \ldots, a^{n}(i)>\in p^{A} i\right\} n$

$n\left\{i \in I: a^{1}(i)=b^{1}(i)\right\} \cap \ldots \cap\left\{i \in I: a^{n}(i)=b^{n}(i)\right\}$,

e como todos os conjuntos do 2? membro pertencem a D segue, pelo fato de ser D um filtro, que:

$\left\{i \in I:\left\langle b^{1}(i), \ldots, b^{n}(i)>p_{i}\right\} \in D\right.$ o que mostra que a definição de $<a_{D}^{1}, \ldots, a_{D}^{n}>\in p^{A}$ independe dos representantes.

3. Se $f$ é un sĭmbolo funcional m-ärio de $L$ e $a_{D}^{1}, \ldots, a_{D}^{m}|A|$, então: $\quad f^{A}\left(a_{D}^{1}, \ldots, a_{D}^{m}\right)=\left\langle f_{i}^{A}\left(a^{\prime}(i), \ldots, a^{m}(i)\right): i \in I\right\rangle_{D} \in|A|$. Analogamente, se $a_{\mathrm{D}}^{1}=b_{\mathrm{D}}^{1}, \ldots, a_{\mathrm{D}}^{\mathrm{m}}=b_{\mathrm{D}}^{\mathrm{m}}$, então: $\left\langle f^{A} i\left(a^{1}(i), \ldots, a^{m}(i)\right): i \in I\right\rangle_{D}=\left\langle f^{A} i\left(b^{1}(i), \ldots, b^{m}(i)\right): i \in I\right\rangle D$ e portanto a definição de $f^{A}\left(a_{D}^{1}, \ldots, a_{D}^{m}\right)$ independe dos re presentantes.

4. Se c é um símbolo de constante de L, então: $c^{A}=\left\langle C A_{i}: i \in I\right\rangle_{D} \in|A|$.

Dada uma famîlia $\left\{s_{i}: V \rightarrow\left|A_{i}\right|:\right.$ ieI $\}$ de substituições va mos denotax por $\left\langle s_{i}: i \epsilon I\right\rangle_{D}$ a substituição $<s_{i}: i \in I>_{D}: V \rightarrow|A|$ tal que $\left\langle s_{i}: i \in I\right\rangle_{D}\left(v_{n}\right)=\left\langle s_{i}\left(v_{n}\right): i \in I\right\rangle_{D}$ para todo $n \in \mathbb{N}$.

Se $D$ e un uItrafiltro o produto reduzido $\underset{i \in I}{\prod_{i}} A_{i} / D$ se $d i z$ um Uetraproduto. Para ultraprodutos demonstraremos um resultado de Los conhecido como o "Teorema fundamental dos U1tra produtos" que fornece condições necessärias e suficientes para que uma fórmula seja välida no ultraproduto a partir 
da validade dela nas estruturas componentes:

TEOREMA III2.1 (LoS) - Sejam A o ultraproduto $\prod_{i \in I} A_{i} / D$ das L-estruturas $\left\{A_{i}: i \in I\right\}$ pelo ultrafiltro $D, \quad\left\{s_{i}: V \rightarrow\left|A_{i}\right|: i \in I\right\}$ uma famîlia de substituições e $s=\left\langle s_{i}: i \in I\right\rangle_{D}: V \rightarrow|A|$. Então:

(i) para todo termo $t \in \operatorname{Ter}(L), \bar{s}(t)=\left\langle\overline{s_{i}}(t): i \in I\right\rangle_{D}$ (isto

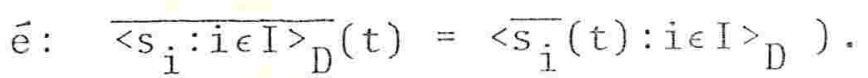

(ii) para toda förmula $A \in F o r(L), A \neq A[S]$ se e só se $\left\{i \in I: A_{i} \vDash A\left[s_{i}\right]\right\} \in D$.

DEMONSTRAÇAO

(i) por indução sobre os termos:

1. se o termo té uma variảvel v, vale a afirmaçäo por definição da $s=\left\langle s_{i}: i \in I\right\rangle_{D}$

2. se o termo $t$ é um símbolo de constante $c$, então

$$
\begin{aligned}
\bar{s}(t) & =\bar{s}(c)=c{ }^{A}=\left\langle c A_{i}: i \in I\right\rangle_{D}=\left\langle\overline{s_{i}}(c): i \in I\right\rangle_{D}= \\
& =\left\langle\overline{s_{i}}(t): i \in I\right\rangle_{D}
\end{aligned}
$$

3. se o termo $t \vec{e} f\left(t_{1} \ldots t_{m}\right)$ onde $f \vec{e}$ un símbolo funcional m-ärio e $t_{1}, \ldots, t_{m}$ termos, então:

$$
\bar{s}(t)=\bar{s}\left(f\left(t_{1} \ldots t_{m}\right)\right)=f^{A}\left(\bar{s}\left(t_{1}\right), \ldots, \bar{s}\left(t_{m}\right)\right)
$$

que por hipötese de indução $\vec{e}$ igual a:

$$
\left.f^{A}\left(\left\langle\overline{s_{i}}\left(t_{I}\right): i \in I\right\rangle_{D}, \ldots, \overline{s_{i}}\left(t_{m}\right): i \in I\right\rangle_{D}\right)
$$

que por definição de $f^{A} \vec{e}$ igual a:

$$
\begin{aligned}
& \left.\left\langle f^{A} \bar{s}_{i}\left(t_{1}\right), \ldots, \overline{s_{i}}\left(t_{m}\right)\right): i \in I\right\rangle_{D}= \\
& =\left\langle\overline{s_{i}}\left(f\left(t_{1} \ldots t_{m}\right)\right): i \in I\right\rangle_{D}=\left\langle\overline{s_{i}}(t): i \in I\right\rangle_{D} .
\end{aligned}
$$

(ii) será demonstrado, por indução sobre förmulas, que 
para toda famîlia $\left\{s_{i}: V \rightarrow\left|A_{i}\right|: i \in I\right\}$ de substituições vale a afirmação do item (ii).

1. $A \neq t_{1}=t_{2}[s]$ se e sö se $\vec{s}\left(t_{1}\right)=\bar{s}\left(t_{2}\right)$ se e sö se $\left\langle\overline{s_{i}}\left(t_{1}\right): i \in I\right\rangle_{D}=\left\langle\overline{s_{i}}\left(t_{2}\right): i \in I\right\rangle_{D}$ se e so se $\left\{i \in I: \overline{s_{i}}\left(t_{1}\right)=\overline{s_{i}}\left(t_{2}\right)\right\} \in D$ se e só se $\left\{i \in I: A_{i} \neq t_{1}=t_{2}\left[s_{i}\right]\right\} \in D$.

2. usando a definição de $p^{A}$ segue analogamente a 1 que: $A \mid=p\left(t_{1} \ldots t_{n}\right)[s]$ se e sö se $\left\{i \in I: A_{i} \neq p\left(t_{1} \ldots t_{n}\right)\left[s_{i}\right]\right\} \in D$.

3. $A \neq T A[s]$ se e só se, $A \not k A[s]$ e isto por hipötese de indução equivale a $\left\{i \in I: A_{i} \vDash A\left[s_{i}\right]\right\} \notin D$ que como $D e ́$ ultrafiltro equivale a $\left\{i \in I: A_{i} \not \neq A\left[s_{i}\right]\right\}=$ $=I-\left\{i \in I: A_{i} \vDash A\left[s_{i}\right]\right\} \in D$ ou seja equivale a $\left\{i \in I: A_{i} \vDash \neg A\left[s_{i}\right]\right\} \in D$.

4. No caso de $A \wedge B$, para proseguir na sequencia de equiva lencias, precisa-se usar que $X \cap Y \in D$ se e só se $X \in D$ e $Y \in D$; e esta $\widehat{e}$ uma consequência imediata das condições (ii) e (iii) de um filtro (nem $\vec{e}$ necessärio usar a condi ção de ultrafiltro). De resto a demonstração é anäloga à de 3 .

5. Se $\left\{i \in I: A_{i} \vDash V_{V_{k}} A\left[s_{i}\right]\right\} \in D$, então: Dado $a_{D}=\left\langle a_{i}: i \in I\right\rangle_{D} \in|A|$, tem-se que $s\left(v_{k} \mid a_{D}\right)$ é precisamente a substituição $\left.<s_{i}\left(v_{k} \mid a_{i}\right): i \in I\right\rangle_{D}$ e portanto, por hipótese de indução, $A \models A\left[s\left(v_{k} \mid a_{D}\right)\right]$ se e só se $\left\{i \in I: A_{i} \vDash A\left[s_{i}\left(v_{k} \mid a_{i}\right)\right]\right\} \subset D$. Mas qualquer que seja 
$a_{D} \in|A|, \quad\left\{i \in I: A_{i} \neq A\left[s_{i}\left(v_{k} \mid a_{i}\right)\right]\right\} \supset\left\{i \in I: A_{i} \vDash \forall v_{k} A\left[s_{i}\right]\right\}$ e portanto $\left\{i \in I: A_{i} \vDash A\left[s_{i}\left(v_{k} \mid a_{i}\right)\right]\right\} \in D$. Logo para todo. $a_{\mathrm{D}} \in|A|, \quad A \vDash A\left[s\left(v_{k} \mid a\right)\right]$, ou seja $A \neq \forall_{k} A[s]$.

Reciprocamente, se $\left.\left\{i \in I: A_{i} \vDash \forall v_{k} A_{\left[s_{i}\right.}\right]\right\} \& D$, então: $x=\left\{i \in I: A_{i} \not \forall \forall v_{k} A\left[s_{i}\right]\right\}=I-\left\{i \in I: A_{i} \vDash \forall v_{k} A\left[s_{i}\right]\right\} \in D$. Para cada $i \in X$, seja $a_{i} \in\left|A_{i}\right|$ tal que $A_{i} \not A_{i}\left[s_{i}\left(v_{k} \mid a_{i}\right)\right]$ e para cada $i \in I-X$ seja $a_{i} \in\left|A_{i}\right|$ um elemento qualquer de $\left|A_{i}\right|$ uma vez que $\left|A_{j}\right| \neq \phi ;$ e seja $a_{D}=\left\langle a_{i}: i \in I\right\rangle D$. Por hipótese de indução $A \models A\left[s\left(v_{k} \mid a_{D}\right)\right]$ se e sö se $\left\{i \in I: A_{i} \vDash A\left[s_{i}\left(v_{k} \mid a_{i}\right)\right]\right\} \in D$. Mas devido a escolha dos $a_{i} s \quad\left\{i \in I: A_{i} \not F A\left[s_{i}\left(v_{k} \mid a_{i}\right)\right]\right\} \supset x$ e portanto pertence a D. $\log 0 \mathrm{~A} \not \neq \mathrm{A}\left[\mathrm{s}\left(\mathrm{v}_{\mathrm{k}} \mid a_{\mathrm{d}}\right)\right]$ ou seja $\mathrm{A} \not \forall \forall \mathrm{v}_{\mathrm{k}} \mathrm{A}[\mathrm{s}]$.

COROLÁRIO - Com as notações do teorema:

$A \models A$ se e só se $\left\{i \in I: A_{i} \vDash A\right\} \in D$.

DEMONSTRACAO

Se $\left\{i \in I: A_{i} \mid F A\right\} \in D$, então dada $s: V \rightarrow|A|$ existem $s_{i}: V \rightarrow\left|A_{i}\right|$ tais que $s=\left\langle s_{i}: i \in I\right\rangle_{D}$ e pelo teorema $A \models A[s]$ pois $\left\{i \in I: A_{i} \vDash A\left[s_{i}\right]\right\} \supset\left\{i \in I: A_{i} \vDash A\right\}$ e portanto per tence a D. Como isto vale para toda $S: V \rightarrow|A|$, segue que $A \neq A$.

Reciprocamente se $\left\{i \in I: A_{i} \vDash A\right\} \& D$, então $X=\left\{i \in I: A_{i} \not F A\right\}=I-\left\{i \in I: A_{i} \neq A\right\} \in D$, para cada $i \in X$, seja $s_{i}: V \rightarrow\left|A_{i}\right|$ tal que $\left.A_{i} \mid \not A s_{i}\right]$ e para $i \in I-X$, seja $s_{i}: V \rightarrow\left|A_{i}\right|$ uma substituição qualquer; e seja $s=\left\langle s_{i}: i \in I\right\rangle_{D}{ }^{\circ}$ 
Pelo teorema $A \forall A[s]$ pois $\left\{i \in I: A_{i} \not \forall A\left[s_{i}\right]\right\} \supset X$ e por tanto pertence a D. $\log O A \not F A$.

Sejam $L=\{*, e, \leq\}$ uma linguagem para grupos ordenados e $L=\{+, \cdot, 0,1\}$ uma linguagem para corpos; e sejam $<\mathrm{G},{ }_{\mathrm{G}}, e_{\mathrm{G}}, \leq_{\mathrm{G}}>$ um grupo ordenado, $<\mathrm{K},{ }_{\mathrm{K}},{ }_{\mathrm{K}}, 0_{\mathrm{K}}, 1_{\mathrm{K}}>$ um corpo e $v: K^{*} \rightarrow G$ uma valoraçäo de $K$ com grupo de valores $G$. Estamos interessados em definir uma 1 inguagem de la. ordem e enunciar nesta linguagem axiomas, de modo que os modelos desta teoria sejam precisamente os corpos valorados -que nos $l^{\text {: }} \mathrm{s}$. capítulos denotävamos por- $\langle\mathrm{K}, \mathrm{V}, \mathrm{G}\rangle$.

Definiremos a linguagem para a teoria dos corpos valorados como sendo $L=\{g r, k, *, e, \leq,+, \cdot, 0,1, u\}$, onde $g r$ e $k$ são dois símbolos de predicados unärios e u $\vec{e}$ um símbolo de função unäria. Para uma L-estrutura tomaremos: $A=\left\langle G \| K, G, K,{ }_{G}, e_{G}, \leq_{G},{ }_{K},{ }_{K}, 0_{K}, 1_{K}, v\right\rangle$, onde $\left.G\right\rfloor K$-a reu nião disjunta de $G$ e $K-$ é o universo $|A|$ da estrutura, as relações unärias $G$ e $K$ são as interpretações $\mathrm{gr}^{A}$ e $\mathrm{k}^{\mathrm{A}}$ dos símbolos de predicado $\mathrm{gr}$ e $k$ na estrutura e a função $v$ ë a interpretação $u^{A}$ do simbolo funcional $u$ na estrutura. A rigor como $v$ não $\vec{e}$ definido em todo $G \perp K$ e ${ }^{*}{ }_{G},{ }_{K},{ }_{K}$ não são definidos em todo 
$\left(G\lfloor K)^{2}\right.$, A não está de acordo com a definição III1.2 de L-estrutura. Mas isto não causarä problemas, pois podemos supor ${ }_{G},{ }^{+}{ }_{K},{ }_{K}$ extendidas, arbiträria e artificialmente a $(G \| K)^{2}$ e $v$ a $G \perp K$.

AXIOMAS PARA A TEORIA DOS CORPOS VALORADOS:

1. $\forall x(g r(x) \leftrightarrow k(x))$ : caracteriza o fato que o universo da estrutura é uma reunião disjunta de $\operatorname{gr}^{A}$ e $k^{A}$.

2. Axiomas para o grupo ordenado $\left\langle\mathrm{G},{ }^{*}{ }_{\mathrm{G}}, e_{\mathrm{G}}, \leq_{\mathrm{G}}\right\rangle$ : Formalmente na estrutura $A, \quad{ }_{G}$ è uma função de $\left(G\lfloor K)^{2}\right.$ em $\mathrm{G}\left\lfloor\mathrm{K}, \quad e_{\mathrm{G}} \in \mathrm{G} \Perp \mathrm{K} \quad \mathrm{e} \quad \leq_{\mathrm{G}} \subset\left(\mathrm{G}\lfloor\mathrm{K})^{2}\right.\right.$. Por isto precisamos adicio nar aos axiomas de grupo ordenado os fatos adicionais sobre ${ }_{G}^{*}, e_{G}, \leq_{G}$; por exemplo:

$\forall x y z(\operatorname{gr}(x) \wedge g r(y) \wedge g x(z) \rightarrow(x * y) * z=x *(y * z)) \quad:$ para a associatividade.

$\forall x y(g r(x) \wedge g r(y) \rightarrow g r(x * y)):$ para garantir que $G$ é fechado em relação a *G.

$g r(e) \wedge \forall x(g r(x) \rightarrow x * e=x \wedge e * x=x)$ : para o elemento neutro $e_{G}$. $\forall_{x y}(\operatorname{gr}(x) \wedge g r(y) \rightarrow x \leq y \vee y \leq x):$ para garantir que a ordem em $G$ seja total.

e assim por diante para todos os axiomas de grupo ordenado.

3. Axiomas para o corpo $<\mathrm{K},{ }_{\mathrm{K}},{ }^{\circ}, 0_{\mathrm{K}}, 1_{\mathrm{K}}>$ :

Escolhidos de modo anälogo ao item 2., por exemplo, para a existência de inverso: $\quad \forall x(k(x) \wedge x \neq 0 \rightarrow \exists y(k(y) \wedge x \cdot y=1 \wedge y \cdot x=1))$ 
4. Axiomas para a valoração $v=u^{A}$ :

$\forall x(k(x) \wedge x \neq 0 \rightarrow g r(u(x)))$ : para garantir que $v$ è uma função de $K^{*}$ com valores em G.

$\forall x(g \wedge(x) \rightarrow \exists y(k(y) \wedge y \neq 0 \wedge x \sqsupset u(y))): v \vec{e}$ sobre G.

$\forall x y(k(x) \wedge k(y) \wedge x \neq 0 \wedge y \neq 0 \rightarrow u(x \cdot y)=u(x) * u(y)):$ propriedade $(i)$

das valorações.

$\forall x y z(k(x) \wedge k(y) \wedge x \neq 0 \wedge y \neq 0 \wedge x+y \neq 0 \rightarrow(u(x) \leq u(y) \rightarrow u(x) \leq u(x+y))) \quad:$

propriedade (ii) das valorações.

Definimos então uma linguagem de la. ordem e sentenças nesta linguagem de tal modo que uma estrutura $A=\langle G\rfloor K, G, K,{ }^{*}, e_{G}, \leq_{G},{ }^{+}{ }_{K},{ }^{\circ}, 0_{K}, I_{K}, v>$ para esta 1 inguagem satisfaz aquelas sentenças se e so se $\langle K, V, G\rangle$ (na notação antiga) $\vec{e}$ um corpo valorado. Vimos assim que a teoria dos corpos valorados pode ser enunciada numa linguagem de 1a. ordem. Para maior comodidade e sem prejuizos para a cla reza, voltaremos a escrever <K,,$G\rangle$ para a estrutura $A$, designar por + tambëm a operação do grupo e por 0 tambëm o elemento neutro do grupo.

Sejam $\left\{A_{i}=\left\langle K_{i}, v_{i}, G_{i}\right\rangle: i \in I\right\}$ uma familia de corpos valorados, D um ultrafiltro sobre $I$ e $A=\underset{i \in I}{\pi} A_{i} / D$ o ultraproduto dos $A_{i}$ pelo ultrafiltro $D$.

Como cada $A_{i}$ satisfaz os axiomas de corpo valorado segue pelo Teorema de Los que A também satisfaz tais axiomas 
e portanto é um corpo valorado. Em particular o universo $|A|$ de $A$ será a reunião disjunta do universo $G=g r{ }^{A}$ de um grupo ordenado com o universo $\mathrm{k}=\mathrm{k}^{\mathrm{A}}$ de um corpo e a função $v=u^{A}$ será uma valoração de $K$ com grupo de valores G. Podemos então dizer que $A \vec{e}$ o corpo valorado $\langle K, V, G\rangle$ de acordo com a convenção feita acima.

Sejam $a_{i} \in G_{i}$ para $i \in I$, o elemento $\left\langle a_{i}: i \in I>\right.$ pertence tanto a $\prod_{i \in I} G_{i}$ quanto a $\prod_{i \in I} G_{i} \| K_{i}$, e o elemento $<a_{i}: i \in I>D$ pode ser visto como um elemento de $\underset{i \in I}{\prod_{i}} G_{i} / D$ ou $\underset{i \in I}{I}\left(G_{i} \perp\left(K_{i}\right) / D\right.$ E apesar que em cada caso ê um objeto diferente (no $1^{\circ}$ caso $\left\langle a_{i}: i \in I\right\rangle_{D}$ é igual a $\left\{\left\langle b_{i}: i \in I>\in \Pi_{i \in I} G_{i}:\left\{i \in I: a_{i}=b_{i}\right\} \in D\right\}\right.$ e no 2 ? e igual a $\left.\left\{<b_{i}: i \in I>\in \underset{i \in I}{\Pi} G_{i} \perp K_{i}:\left\{i \in I: a_{i}=b_{i}\right\} \in D\right\}\right)$ podemos

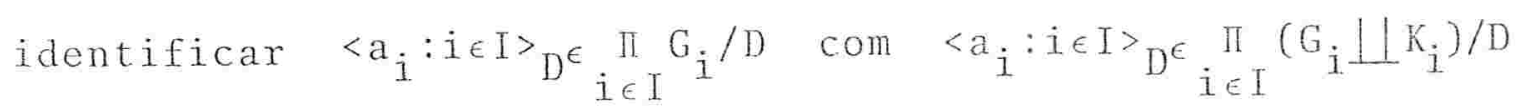
(tambëm pois para $<a_{i}: i \in I>e<a_{i}: i \in I>\epsilon \prod_{i} G_{i}$, vale que: $\left\langle a_{i}: i \in I\right\rangle_{D}=\left\langle a_{i}^{-}: i \in I\right\rangle_{D}$ no $I^{\circ}$ caso se e só $s e\left\langle a_{i}: i \in I\right\rangle_{D}=\left\langle a_{i}^{-}: i \in I\right\rangle_{D}$ no 2 caso) e encarar $\prod_{i \in I} G_{i} / D$ como um subconjunto de $\prod_{i \in I}\left(G_{i} \perp K_{i}\right) / D$. Desta forma $\prod_{i \in I} G_{i} / D$ será precisamente $G$ pe 10 item 2, da definição de ultraproduto de estruturas (defi ni.ção III2.2)

Da mesma forma "encarando" $\prod_{i \in I} K_{i} / D$ como subconjunto de $\prod_{i \in I}\left(G_{i} \perp K_{i}\right) / D$, temos que $K=\prod_{i \in I} K_{i} / D$, e as igualdades: $G=\underset{i \in I}{\prod_{i}} G_{i} / D$ e $K=\prod_{i \in I} K_{i} / D$ valem, não apenas entre os conjun- 
tos, mas também entre as estruturas de grupo ordenado e corpo de quem estes conjuntos são os universos.

Seja $\quad a_{D}=\left\langle a_{i}: i \in I>{ }_{D} \in R_{<K, v>} \subset K\right.$, isto significa que $a_{D} \in K$ e $v\left(a_{D}\right) \geq 0$ e portanto que $\left\{i \in I: v_{i}\left(a_{i}\right) \geq 0_{i}\right\} \in D$ que equivale a $\left\{i \in I: a_{i} \in R_{<K_{i}}, v_{i}>\in D\right.$. Temos então que $R_{<K}, v>=\prod_{i \in I} R_{\left\langle K_{i}\right.}, v_{i}>/ D$ onde estamos mais uma vez "encarando" $\underset{i \in I}{I I} \mathrm{R}_{<K_{i}}, v_{i}>/ D$ como un subconjunto de $K=\prod_{i \in I} K_{i} / D$.

Analogamente $I_{\langle K, v\rangle}=\underset{i \in I}{I I} I_{\left\langle K_{i}, v_{j}>\right.} / D$. Vale tambëm que:

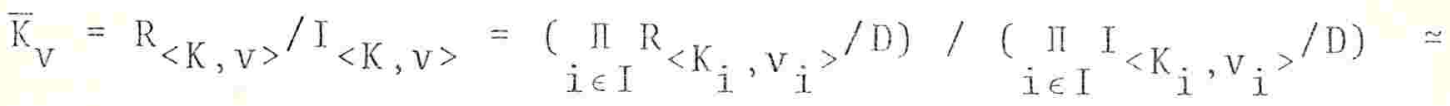

$$
\begin{aligned}
& \simeq \prod_{i \in I}\left(R_{<K_{i}}, v_{i}>/ I_{<K_{i}, v_{i}}\right) / D=\pi_{i \in I}\left(\overline{K_{i}}\right)_{v_{i}} / D \text {, pois não ë di- }
\end{aligned}
$$

fícil de ver que, a aplicaçäo que a $a_{\mathrm{D}}+\mathrm{I}_{<\mathrm{K}, \mathrm{v}\rangle} \in \overline{\mathrm{K}}_{\mathrm{v}} \quad$ com $a_{D} \in R_{\langle K, v>}, \quad$ associa $\left\langle a_{i}{ }^{+I}\left\langle K_{i}, v_{i}>i \in I>D^{\in} \underset{i \in I}{\pi}\left(\overline{K_{i}}\right)_{v_{i}} / D, \quad\right.\right.$ onde $a_{D}=<a_{i}: i \in I>D \quad$ com $a_{i} \in R_{<K_{i}, v_{i}}>$ para todo $i \in I$, está bem definida e é um isomorfismo de corpos.

Sejam agora, para cada primo positivo $p, A_{p}=\left\langle Q_{p}, v_{p}, \mathbb{Z}\right\rangle$ e $A_{p}^{\prime}\left\langle\mathbb{Z}_{p}((X))\right.$, ord, $\left.\mathbb{Z}\right\rangle$ os completados de $\left\langle Q, v_{p}, \mathbb{Z}\right\rangle$ e $<\mathbb{Z}_{\mathrm{p}}(X), v_{X}, \mathbb{Z}>$ (veja seçäo I4) e seja D um ultrafiltro não principal do conjunto dos nümeros primos positivos P. Os ultraprodutos $A=\underset{p \in P}{\operatorname{II}} A_{p} / D$ e $A^{\prime}=\underset{p \in P}{\operatorname{II}} A_{p}^{\prime} / D$ são dois corpos va lorados $A=\langle K, v, G\rangle$ e $A^{\prime}=\left\langle K^{\prime}, V^{\prime}, G^{\prime}\right\rangle$ com $G^{\prime}=G^{\prime}=\underset{p \in P}{\text { II }} \mathbb{Z} / D$ 
(pois $G_{p}=G_{p}^{\prime}=\mathbb{Z}$ para todo $p \in P$ ) e $\bar{K}_{V} \simeq \bar{K}_{V^{\prime}} \simeq \underset{p \in P}{\mathbb{Z}} \prod_{p} / D$ (nois $\left.\left(\overline{K_{p}}\right)_{v_{p}}=\left(\overline{Q_{p}}\right)_{v_{p}} \simeq \mathbb{Z}_{p} \simeq \overline{\mathbb{Z}_{p}((X))_{\text {ord }}}=\left(\overline{K_{p}^{\prime}}\right)_{v_{p}^{\prime}}\right)$

Além disso $\operatorname{car}\left(\bar{K}_{V}\right)=\operatorname{car}\left(\bar{K}_{V^{\prime}}^{\prime}\right)=0$, pois para todo $p^{\prime} \in P$ a sentença $\mathrm{p}^{\prime} \cdot 1=0 \quad$ é falsa no ultraproduto $\underset{\mathrm{p} \in \mathrm{P}}{\prod_{\mathrm{Z}}} \mathrm{p} / \mathrm{D}$ uma vez que $\left\{p \in P: \mathbb{Z}_{p} \mid p^{\prime} \cdot 1=0\right\}=\left\{p^{\prime}\right\} \notin D$ jä que $D$ é um ultrafiltro não principal. Portanto $\langle K, v, G\rangle$ e $\left\langle K^{\prime}, V^{\prime}, G^{\prime}\right\rangle$ são ambos não ramificados, com indice de ramificação zero.

Mostrando que $\langle K, v, G\rangle$ e $\left\langle K^{\prime}, v^{\prime}, G^{\prime}\right\rangle$ possuem $x$-seções, satisfazem o Lema de Hense1, são w-pseudocompletos e que a cardinalidade de $K$ e $K^{\prime} \quad \vec{e} t_{1}$, estarão satisfeitas to das as condições para o Teorema II2, e poderemos então concluir:

TEOREMA III3.1 - Existe um isomorfismo x-analitico entre os ultraprodutos $A=\underset{p \in P}{I}<Q_{p}, v_{p}, \mathbb{Z}>/ D$ e $A^{\prime}=\underset{p \in P}{\operatorname{II}}<\mathbb{Z} p((X))$, ord, $\mathbb{Z}>/ D, \quad$ onde $D$ è um ultrafiltro näo principal de P. DEMONSTRAÇÃO

As duas primeiras afirmações -que $A$ e $A^{\prime}$ possuem $x$-seções e satisfazem o Lema de Hense1- seräo consequências do Teorema de $Ł o s$, desde que se mostre que tais afirmaçoes podem ser enunciadas em linguagem de 1 a. ordem.

1. Seja $L^{\prime}=L u\{\pi\}$, onde $L$ é a 1 inguagem para corpos valorados e $\pi$ é um símbolo funcional unärio; e sejam $B_{p}$ 
e $B_{p}^{\prime}$ as L'-estruturas obtidas acrescentando-se a $A_{p}=\left\langle Q_{p}, v_{p}, \mathbb{Z}\right\rangle$ e $A_{p}^{\prime}=\left\langle\mathbb{Z}{ }_{p}((X))\right.$, ord, $\left.\mathbb{Z}\right\rangle$ as $x$-seções (que se sa be que estes dois corpos valorados possuem) $\pi^{B} p$ e $\pi^{B} p$ para a interpretação do simbolo funcional unärio $\pi$ em $B_{p}$ e $B_{p}^{\prime}$ (aqui tambëm supõe-se $\pi^{B} p$ e $\pi^{B} p_{p}^{\prime}$ extendidas arbi traria e artificialmente a todo $G_{p} \| K_{p}$ e $\left.G_{p}^{\prime} \Perp K_{p}^{\prime}\right)$. As es truturas $B_{p}$ e $B_{p}^{\prime}$ satisfarão então as seguintes senten ças de $L^{\prime}$ :

$\forall x(g r(x) \rightarrow k(\pi(x)))$ : para garantir que $\pi$ é uma função de $G$ em K.

$\forall x y(g r(x) \wedge g r(y) \rightarrow \pi(x * y)=\pi(x) \cdot \pi(y)): \pi$ e homomorfismo. $\forall x(g x(x) \rightarrow u(\pi(x))=x): \quad v \circ \pi=i d_{G}$.

e portanto (pelo teorema de Los) os ultraprodutos:

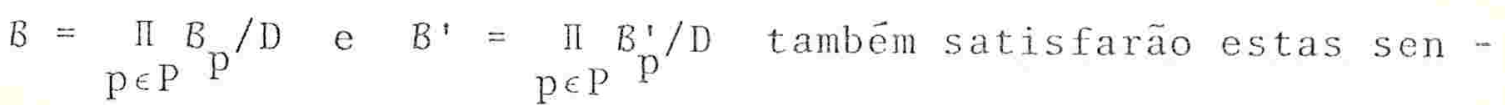
tenças. Mas isto quer dizer que $\pi^{B}$ e $\pi^{B^{\prime}}$-interpreta çöes do símbolo $\pi$ nas estruturas $B$ e $B^{\prime}-$ são $x$-seções para os corpos valorados $A$ e $A^{\prime}$. E como $\operatorname{car}\left(\bar{K}_{V}\right)=$ $=\operatorname{car}\left(\overline{K_{V}^{\prime}},\right)=0$ estas $x$-seções são automaticamente normalizadas.

2. Para cada $n>0$ e $0<m<n$, mostraremos como escre ver uma sentença na linguagem de 1 a. ordem para os corpos valorados (em notação simplificada) tal que um corpo valora do $\langle K, v, G\rangle$ satisfarả esta sentença se e sö se:

Qualquer que sejam $f, g, h \in R_{<K, v>}[X]$, se $\operatorname{gr}(f)=n$ e $g r(g)=g r(\bar{g})=m \quad$ e $g r(h) \leq n-m \quad$ e $\bar{f}=\bar{g} \cdot \bar{h} \quad$ e $\bar{g}, \bar{h} \quad$ são relativa 
mente primos, então existem $g^{*}, h^{*} \in R_{\langle K, v\rangle}[X]$ tais que $\operatorname{gr}\left(g^{*}\right)=\operatorname{gr}(g)$ e $\overline{g^{*}}=\bar{g}$ e $\overline{h^{*}}=\bar{h}$ e $\quad f=g \cdot h$.

E claro que $\langle K, v, G\rangle$ satisfarä todas estas sentenças quando variam $n$ e $m$ se e só se ele satisfizer o Lema de Hense1.

Começamos quantificando $\forall x_{0} \ldots x_{n} y_{0} \cdots y_{m} z_{0} \cdots z_{n-m}$ (onde $x_{0}, \ldots, z_{n-m}$ são $2 n+3$ variäveis distintas) para descre ver os polinômios $f=x_{0}+x_{1} x+\ldots+x_{n} x^{n}, g=y_{0}+y_{1} x+\ldots+y_{m} x^{m}$ e $h=z_{0}+\ldots+z_{n-m} x^{n-m}$. Depois colocamos $v\left(x_{0}\right) \geq 0 \wedge \ldots \wedge v\left(x_{n}\right) \geq 0$ $\wedge v\left(y_{0}\right) \geq 0 \wedge \ldots \wedge v\left(z_{n-m}\right) \geq 0$ (onde $v(a) \geq \alpha$ abrevia a fórmula: $(a \neq 0 \wedge v(a) \geq a) \vee a=0 ;$ e a mesma observação valendo para $v(a)>a)$ para descrever que $f, g, h \in R_{<K, v}[X], \quad$ e $x_{n} \neq 0 \wedge v\left(y_{m}\right)=0$

para garantir que $g r(f)=n$ e $g r(g)=g r(\bar{g})=m$. Enunciamos que $\bar{f}=\bar{g} \cdot \bar{h}$ atravês de: $\quad v\left(x_{0}-y_{0} z_{0}\right)>0 \wedge v\left(x_{1}-y_{0} z_{1}-y_{1} z_{0}\right)>0 \wedge$ $\wedge \ldots \wedge v\left(x_{n-1}-y_{m-1} z_{n-m^{-}} y_{m}^{z} n-m-1\right)>0 \wedge v\left(x_{n}-y_{m}^{z} n-m\right)>0 \quad(o b-$ serve que dados os inteiros $n$ e $m$ podemos escrever "expli citamente" -sem o uso dos pontos- esta expressão).

$\overline{\mathrm{g}}, \overline{\mathrm{h}}$ relativamente primos equivale a: existem $\overline{\mathrm{a}}, \overline{\mathrm{b}} \in \overline{\mathrm{K}}_{\mathrm{V}}[\mathrm{X}]$ tais que $\overline{\mathrm{a}} \cdot \overline{\mathrm{g}}+\overline{\mathrm{b}} \cdot \overline{\mathrm{h}}=1$ e $\operatorname{gr}(\overline{\mathrm{a}})<\mathrm{n}-\mathrm{m}$ e $\operatorname{gr}(\overline{\mathrm{b}})<\mathrm{m} \quad$ (pois, pe10 Teorema de Bezout, existem $\bar{a}_{1}, \bar{b}_{1} \in \bar{K}_{V}[X]$ tais que $\bar{a}_{1} \bar{g}+\bar{b}_{1} \bar{h}=1$ e portanto $\left(\bar{a}_{1}-\overline{\lambda h}\right) \bar{g}+\left(\bar{b}_{1}+\overline{\lambda g}\right) \bar{h}=1$ para todo $\bar{\lambda} \epsilon_{V}[X]$ Tomando para $\bar{\lambda}$ o quociente de $\bar{a}_{1}$ por $\bar{h}$ teremos que: $\operatorname{gr}\left(\bar{a}_{1}-\overline{\lambda h}\right)<\operatorname{gr}(\bar{h}) \leq n-m$ e $\left.\operatorname{gr}\left(\bar{b}_{1}+\overline{\lambda g}\right)<\operatorname{gr}(\bar{g})=m\right)$. E isto pode ser escrito em linguagem de 1 a. ordem usando o mesmo tipo de procedimento que anteriormente, uma vez que os graus 
de $\bar{a}$ e $\bar{b}$ estão limitados por números fixados.

E assim por diante pode-se escrever sem mais dificuldades o restante das condições para a sentença.

os corpos valorados $\left\langle Q_{p}, v_{p}, \mathbb{Z}>\right.$ e $\left\langle\mathbb{Z}_{p}((X))\right.$, ord, $\left.\mathbb{Z}\right\rangle$ são completos e portanto satisfazem o Lema de Hensel (teor. I4.1) Como o Lema de Hensel é enunciäve1 na linguagem de 1a. ordem para corpos valorados segue pelo Teorema de Los que os ultra produtos $\underset{\mathrm{p} \in \mathrm{P}}{\Pi}<\mathrm{Q}_{\mathrm{p}}, v_{\mathrm{p}}, \mathbb{Z}>/ \mathrm{D} \quad \mathrm{e} \underset{\mathrm{p} \in \mathrm{P}}{\prod_{\mathrm{P}}<\mathbb{Z}_{\mathrm{p}}}((\mathrm{X}))$, ord, $\mathrm{Z}>/ \mathrm{D} \quad$ tambëm satisfazem o Lema de Hense1.

3. Não se pode enunciar na 1inguagem de 1 a. ordem para corpos valorados que um corpo ê $\omega$-pseudocompleto. De modo que não se pode concluir, usando o Teorema de Los como nos casos precedentes, que um ultraproduto é $\omega$-pseudocompleto a partir de w-pseudocompletude dos corpos componentes.

Mas neste caso a w-pseudocompletude dos dois ultraprodutos será consequência apenas do fato de ser o ultraproduto não principal e sobre uma famîlia enumerável de corpos valorados cujos grupos de valores admitem um minimo positivo.

Vamos então demonstrar o seguinte:

LEMA III3.1 - Sejam $\left\{A_{i}=<K_{i}, v_{i}, G_{i}>: i \in \omega\right\}$ uma família enumerävel de corpos valorados com $G_{i}=\mathbb{Z}$ para todo $i \in \omega$, e seja D um ultrafiltro não principal sobre w. Então: 
$\langle K, v, G\rangle=A=\prod_{i \in \omega} A_{i} / D \quad \vec{e} \quad \omega$-pseudocompleto.

\section{DEMONSTRAÇÃO}

Seja $\left\{a_{D}^{n}\right\}_{n<\omega}$ uma sequência pseudoconvergente de $\langle K, v, G\rangle$. Isto significa que existe $n_{0}<\omega$ tal que $v\left(a_{D}^{S}-a_{D}^{r}\right)<v\left(a_{D}^{t}-a_{D}^{S}\right)$ para todos $n_{0} \leq r<s<t<\omega$ e que a sequëncia $\gamma_{D}^{n}=v\left(a_{D}^{n+1}-a_{D}^{n}\right)$ é estritamente crescente a partir de $n_{0}$ (veja definição I5.I e a demonstração do Lema I5.1).

Sejam $\gamma_{i}^{n}=v_{i}\left(a_{i}^{n+1}-a_{i}^{n}\right) \in G_{i}$ para todos $n, i \in \omega$. Por definição de ultraproduto, $\gamma_{D}^{n}=\left\langle\gamma_{i}^{n}: i \epsilon \omega\right\rangle_{D}$ e para todo $n \geq n_{D}$, $x_{n}=\left\{i \in \omega: \gamma_{i}^{n}<\gamma_{i}^{n+1}\right\} \in D$.

A partir destes fatos é possivel construir para cada $i \in \omega$ uma sequência $\left\{\delta_{i}^{n}\right\}_{n}$ de elementos de $G_{i}$ tais que:

$\delta_{D}^{n}=\left\langle\delta_{i}^{n}: i \in \omega\right\rangle_{D}=\left\langle\gamma_{i}^{n}: i \in \omega\right\rangle_{D}=\gamma_{D}^{n}$ para todo $n<\omega$ e $\delta_{i}^{n}<\delta_{i}^{n+1}$ para todo $n_{0} \leq n<\omega$ e para todo $i \in \omega$.

Isto será feito, fixado i $\in \omega$, indutivamente sobre $n<\omega$, da seguinte maneira:

$$
\begin{array}{ll}
\operatorname{para} & n \leq n_{0}, \quad \delta_{i}^{n}=\gamma_{i}^{n} \\
\text { para } & n \geq n_{0}, \quad \delta_{i}^{n+1}=\max \left\{\delta_{i}^{n}+1, \gamma_{i}^{n+1}\right\} .
\end{array}
$$

E öbvio então que $\delta_{i}^{n}<\delta_{i}^{n+1}$ para todo $n \geq n_{0}$ e $i \in \omega$ e que $\left\langle\delta_{i}^{n}: i \epsilon \omega\right\rangle_{D}=\left\langle\gamma_{i}^{n}: i \epsilon \omega\right\rangle_{D}$ para todo $n \leq n_{0}$. Seja agora $\left\langle\hat{o}_{i}^{n}: i \in \omega\right\rangle_{D}=\left\langle\gamma_{i}^{n}: i \epsilon \omega\right\rangle_{D}$ para algum $n \geq n_{0}$. Então: 
$Y_{n}=\left\{i \in \omega: \delta_{i}^{n}=\gamma_{i}^{n}\right\} \in D$ e se $i \in X_{n} \cap Y_{n}$, entäo

$\delta_{i}^{n}=\gamma_{i}^{n}<\gamma_{i}^{n+1}$ ou $\delta_{i}^{n}+1 \leq \gamma_{i}^{n+1}, \quad$ donde

$\delta_{i}^{n+1}=\max \left\{\delta_{i}^{n+1}, \gamma_{i}^{n+1}\right\}=r_{i}^{n+1}$ e $Y_{n+1}=\left\{i \in I: \delta_{i}^{n+1}=Y_{i}^{n+1}\right\}_{\supset}$

$\supset X_{n} \cap Y_{n}$. Como $X_{n}, Y_{n} \in D$ e $D$ è um filtro segue que

$X_{n} \cap Y_{n} \in D$ e portanto $Y_{n+1} \in D, \quad$ donde

$\left\langle\delta_{i}^{n+1}: i \in \omega\right\rangle_{D}=\left\langle\gamma_{i}^{n+1}: i \in \omega\right\rangle_{D}$. E assim foi demonstrado, por indução, que: $\quad \delta_{D}^{n}=\left\langle\delta_{i}^{n}: i \in \omega\right\rangle_{D}=\left\langle\gamma_{i}^{n}: i \in \omega\right\rangle_{D}=\gamma_{D}^{n}$ para todo $n \in w$.

Agora, para cada iew, serä construida uma sequência $\left\{b_{i}^{n}\right\}_{\in} \omega$ de elementos de $k_{i}$ tal que:

$b_{D}^{n}=\left\langle b_{i}^{n}: i \in \omega\right\rangle_{D}=\left\langle a_{i}^{n}: i \in \omega\right\rangle_{D}=a_{D}^{n}$ para todo $n \in \omega$ e

$v_{i}\left(b_{i}^{n+1}-b_{i}^{n}\right)=\delta_{i}^{n}$ para todos $n_{0} \leq n<\omega$ e $i \in \omega$.

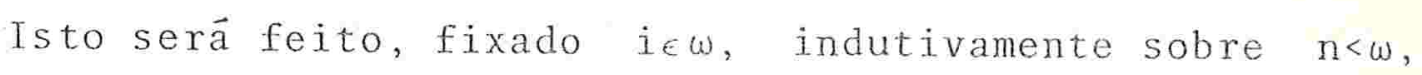
da seguinte forma:

$$
\begin{array}{ll}
\text { para } n \leq n_{0}, & b_{i}^{n}=a_{i}^{n} \\
\text { para } n \geq n_{0}, & b_{i}^{n+1}= \begin{cases}a_{i}^{n+1} \text { se } v_{i}\left(a_{i}^{n+1}-b_{i}^{n}\right)=\delta_{i}^{n} \\
b_{i}^{n}+c_{i}^{n} \text { se } v_{i}\left(a_{i}^{n+1}-b_{i}^{n}\right) \neq \delta_{i}^{n}\end{cases}
\end{array}
$$

onde $c_{i}^{n} \vec{e}$ un elemento de $k_{i}$ tal que $v_{i}\left(c_{i}^{n}\right)=\delta_{i}^{n}$.

E öbvio entäo que $v_{i}\left(b_{i}^{n+1}-b_{i}^{n}\right)=\delta_{i}^{n}$ para todo $n \geq n_{0}$ e $i \in \omega$ e que $\left\langle b_{i}^{n}: i \in \omega\right\rangle_{D}=\left\langle a_{i}^{n}: i \in \omega\right\rangle_{D}$ para todo $n \leq n_{0}$. 
Seja agora $\left\langle b_{i}^{n}: i \in \omega\right\rangle_{D}=\left\langle a_{i}^{n}: i \in \omega\right\rangle_{D}$ para algum $n \geq n_{0}$.

Então $z_{n}=\left\{i \in \omega: b_{i}^{n}=a_{i}^{n}\right\} \in D$ e se $i \in Y_{n} n Z_{n}$ então:

$v_{i}\left(a_{i}^{n+1}-b_{i}^{n}\right)=v_{i}\left(a_{i}^{n+1}-a_{i}^{n}\right)=\gamma_{i}^{n}=\delta_{i}^{n}$ e por construção

$b_{i}^{n+1}=a_{i}^{n+1}$. Isto $\vec{e} \quad z_{n+1}=\left\{i \in \omega: b_{i}^{n+1}=a_{i}^{n+1}\right\}>Y_{n} n z_{n}$

e portanto $Z_{n+1} \in D$ o que significa que:

$\left\langle b_{i}^{n+1}: i \in \omega\right\rangle_{D}=\left\langle a_{i}^{n+1}: i \in \omega\right\rangle_{D}$. E assim foi demonstrado, por inđução que $\quad b_{D}^{n}=\left\langle b_{i}^{n}: i \in \omega\right\rangle_{D}=\left\langle a_{i}^{n}: i \in \omega\right\rangle_{D}=a_{D}^{n}$ para todo $n<\omega$.

As sequências $\left\{b_{i}^{n}\right\}_{n \in u}$ são pseudoconvergentes (em $K_{i}$ ) e para todo $n \geq n_{0}$ e $i \in \omega$ vale que: $\delta_{i}^{n}=v_{i}\left(b_{i}^{n+1}-b_{i}^{n}\right)=$ $=v_{i}\left(b_{i}^{r}-b_{i}^{n}\right)$ para todo $r>n$ (veja Lema I5.1).

Sejam então $a_{i}=b_{i}^{i} \in K_{i}$ para cada $i \in \omega . \quad 0$ elemento $a_{D}=\left\langle a_{i}: i \in \omega\right\rangle_{D}$ é um pseudolimite de $\left\{a_{D}^{n}\right\}_{n \in \omega}$ pois:

se $n \geq n_{0}$, então $v\left(a_{D}-a_{D}^{n}\right)=v\left(a_{D}-b_{D}^{n}\right)=\left\langle v_{i}\left(a_{i}-b_{i}^{n}\right): i \epsilon \omega\right\rangle_{D}=$ $<v_{i}\left(b_{i}^{i}-b_{i}^{n}\right): i \in \omega>D$. Como para $r>n \quad v_{i}\left(b_{i}^{r}-b_{i}^{n}\right)=\delta_{i}^{n}$, segue que para $i>n \quad v_{i}\left(b_{i}^{i}-b_{i}^{n}\right)=\delta_{i}^{n} \quad$ ou que $w_{n}=\left\{i \in \omega: v_{i}\left(b_{i}^{i}-b_{i}^{n}\right)=\delta_{i}^{n}\right\} \supset\{i \in \omega: i>n\} . W_{n}$ e um subcon junto de $\omega$ de complementar finito, pertence portando a D, pois D é um ultrafiltro não principal sobre $\omega$. ( Se D é um ultrafiltro não principal sobre um conjunto I e $X=\left\{x_{0}, \ldots, x_{n}\right\} \quad I$, então $I-\left\{x_{0}\right\}, \ldots, I-\left\{x_{n}\right\} \in D$ pois sendo $D$ não principal $\left\{x_{0}\right\}, \ldots,\left\{x_{n}\right\} \& D$ e portanto $I-X=$ $=\left(I-\left\{x_{0}\right\}\right) \ldots\left(I-\left\{x_{n}\right\}\right) \in D \cdot O$ que mostra que os subconjuntos 
de I de complementar finito pertencem a D).

$\log 0\left\langle v_{i}\left(b_{i}^{i}-b_{i}^{n}\right): i \in \omega\right\rangle_{D}=\left\langle\delta_{i}^{n}: i \in \omega\right\rangle_{D}=\left\langle\gamma_{i}^{n}: i \in \omega\right\rangle_{D}=\gamma_{D}^{n}$,

ou $\quad v\left(a_{D}-\frac{n}{D}\right)=\gamma_{D}^{n}$ o que de acordo com o Lema 15.1 mostra que $a_{D}$ é um pseudolimite de $\left\{a_{D}^{n}\right\}$ new , e que $A=\langle K, v, G\rangle$ é $\omega$-pseudocompleto.

Este Lema è suficiente para demonstrar que os ultraprodutos $\underset{p \in P}{\Pi}<Q_{p}, v_{p}, \mathbb{Z}>/ D$ e $\underset{p \in P}{\Pi}<\mathbb{Z} \underset{p}{ }((X))$, ord, $\mathbb{Z}>/ D$ são w-pseudo completos pois ele vale tambëm se o conjunto de indices ë enu merävel (sỏ usamos, como conjunto de índices, $\omega$ no Lema pa ra simplificar a notação na escolha $a_{i}=b_{i}^{i}$, senão teríamos que tomar $a_{i}=b_{i}^{\phi}(i)$, onde $\phi$ seria a bijeção de I sobre $\omega$, e a demonstração seguiria igual).

4. Cada um dos $Q_{p}$ e $\mathbb{Z}_{p}((X))$ tem cardinalidade $2^{x_{0}}$ (veja os comentärios no final da seção I4. onde $Q_{p}$ e $\mathbb{Z}_{p}((X))$ são dados a partir de sêries como consequência da pro posição I4.1) e portanto os produtos cartesianos $\prod_{p \in P} Q_{p}$ e

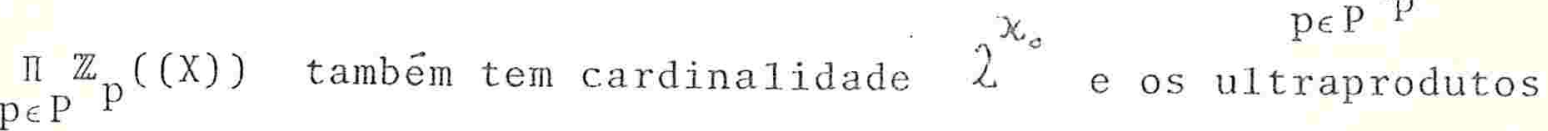
$\prod_{\mathrm{p} \in \mathrm{P}} \mathrm{Q}_{\mathrm{p}} / \mathrm{D} \quad \mathrm{e} \underset{\mathrm{p} \in \mathrm{P}}{\Pi_{\mathbb{Z}}}((\mathrm{X})) / \mathrm{D}$ terão então cardinalidade $\leq 2^{\lambda_{0}}$ Mostrando que eles também tem cardinalidade $\geqslant 2^{x_{0}}$ segui$2^{\lambda_{x_{0}}}$ e pela hipótese do continuo que ela e igual a $x_{1}$. 
LEMA III3.2 - Sejam I um conjunto enumerảvel, D um ultra filtro não principal sobre $I$ e $\left\{A_{i}: i \in I\right\}$ uma familia de con juntos infinitos. Então a cardinalidade de $\prod_{i \in I} A_{i} / D \vec{e} \geqslant 2^{x_{0}}$ DEMONSTRAÇÃO

Seja $I=\left\{x_{0}, x_{1}, \ldots, x_{n}, \ldots\right\}$ uma enumeração de $I$ e sejam $X=I, \quad X_{n+1}=X_{n}-\left\{x_{n}\right\}$ uma sequênica de subconjuntos de $I$ construida indutivamente.

Então $x_{n+1}=I-\left\{x_{0}, \ldots, x_{n}\right\} \in D$ uma vez que o comple mentar de $x_{n+1}$ é finito e $D$ e não principal (isto foi visto durante a demonstração do Lema anterior) e $x_{n} \supset x_{n+1}$ para todo $n \in \omega$ e $n_{n \in \omega} x_{n}=\phi$.

Dado $i \in I$, existe $n \in w$ tal que $i \notin X_{n}$ (uma vez que $n_{n \in \omega} x_{n}=\phi f$, como $i \in X_{0}=I$ segue que existe $k(i) \in \omega$ tal que i $\epsilon x_{k(i)}-x_{k(i)+1}$.

o conjunto $2^{k(i)}$ que é o conjunto de todas as fun ções de $\{0,1, \ldots, k(i)-1\}$ em $\{0,1\}$ - $\vec{e}$ finito (contem $2^{k(i)}$ elementos) e como $A_{i}$ é infinito, existe alguma função inje tora $F_{i}: 2^{k(i)}+A_{i}$.

$2^{\omega}$ é o conjunto de todas as funções de $\omega$ em $\{0,1\}$. Se for possivel definir-se uma função injetora $F: 2 \rightarrow \prod_{i \in I} A_{i} / D$, então estarả demonstrado que a cardinalidade de $2^{\omega}$-que $\vec{e}$ $2^{x_{3}}$ - é menor ou igual que a cardinalidade de $\prod_{i \in I} A_{i} / D$. 
De fato dada $s \in 2^{\omega}$, s restrita a $\{0,1, \ldots k(i)-1\}$ é um $\underline{e}$ lemento de $2^{k(i)}$ (vamos usar a notação $\{0,1, \ldots, k(i)-1\}=$ $\left.=k(i) ; \quad \operatorname{assim} s \mid k(i) \in 2^{k(i)}\right), \quad e \quad F_{i}(s \mid k(i)) \in A_{i} \cdot \operatorname{Seja}$ então: $\quad F(s)=\left\langle F_{i}(s \mid k(i)): i \in I\right\rangle_{D} \epsilon \prod_{i \in I} A_{i} / D$.

Sejam $s, t \in 2^{\omega}$, słt. Então existe $n \in \omega$ tal que $s(n) \neq t(n)$ e se $i \in I$ é tal que $k(i)>n$, então $s / k(i) \neq t / k(i)$ (pois $n \in k(i)$ e $s|k(i)(n) \neq t| k(i)(n) \quad$ ) portanto $F_{i}(s \mid k(i)) \neq F_{i}(t \mid k(i))$ pois $F_{j}$ e injetora. Logo $\left\{i \in I: F_{i}(s \mid k(i)) \neq F_{i}(t \mid k(i))\right\} \supset\{i \in I: k(i)>n\}$.

Mas, se $i \in X_{n+1}$, então $k(i) \geq n+1$ (por definição de $k(i)$ e pelo fato que a sequência $x_{n}$ é decrescente). Logo $x_{n+1} \subset\{i \in I: k(i)>n\}$ e portanto $\{i \in I: k(i)>n\} \in D \quad$ e $\left\{i \in I: F_{i}(s \mid k(i)) \neq F_{i}(t \mid k(i))\right\} \in D$. E isto significa que:

$$
F(s)=\left\langle F_{i}(s \mid k(i)): i \in I\right\rangle_{D} \not^{t}\left\langle F_{i}(t \mid k(i)): i \in I\right\rangle_{D}=F(t)
$$

o que mostra que F è injetora.

Com isto estä terminada a demonstração teorema o qual nos garante a existência de um isomorfismo analítico entre os cor pos valorados $A=\underset{p \in P}{I I}\left\langle Q_{p}, v_{p}, \mathbb{Z}>/ D\right.$ e $A^{\prime}=\underset{p \in P}{I}\left\langle\mathbb{Z}_{p}((X))\right.$, ord, $\left.\mathbb{Z}\right\rangle$ /D sempre que $D$ e um ultrafiltro não principal de $P$, que - de acordo com as definições I1.2 (de isomorfismo analítico) e III 1.3 (de isomorfismo entre estruturas)-- é tambëm um iso- 
morfismo entre as estruturas $A$ e $A^{\prime} ;$ e portanto $A$ e $A^{\prime}$ são elementarmente equivalentes (proposição III1.2), isto é: se A é uma sentença da linguagem de la. ordem para corpos valorados, então A satisfaz A se e sỏ se A' satisfaz A.

Voltando aos corpos $A_{p}=\left\langle Q_{p}, v_{p}, \mathbb{Z}\right\rangle$ e $A_{p}^{\prime}=\langle\mathbb{Z} p((X))$, ord, $\mathbb{Z}>$ podemos extrair o seguinte:

COROLARIO 1 - Seja A uma sentença da linguagem de 1a. ordem para corpos valorados e seja I(A) o conjunto de todos os primos positivos $\mathrm{p} \in \mathrm{P}$ para os quais $\mathrm{A}_{\mathrm{p}}=\left\langle\mathrm{Q}_{\mathrm{p}}, \mathrm{v}_{\mathrm{p}}, \mathbb{Z}\right\rangle$ satis faz $A$ se e só se $A_{p}^{\prime}=\left\langle\mathbb{Z}_{p}((X))\right.$, ord, $\left.\mathbb{Z}\right\rangle$ satisfaz A. Então o complementar P-I(A) de I(A) é finito.

DEMONSTRACAO

Seja $I(A)=\left\{p \in P: A_{p} \vDash A\right.$ se e söse $\left.A_{p}^{\prime} \vDash A\right\}$ e seja $X=P-I(A) \quad$ o complementar de $I(A)$.

Se $X$ fosse infinito, então $X$ pertenceria a algum ultrafiltro näo principal D de P.

Pelo teorema anterior $\underset{p \in P}{\prod_{p}} / D \vDash A$ se e só se

\& Seja $F=\{Y \subset P: P-Y$ e finito $\}$ o filtro de Frëchet de P. O conjunto $F u\{X\}$ satisfaz a propriedade da interseção finita e portan to pela prop.III2.I o filtro $[F u\{X\}]$, gerado por Fu $\{X\}$, é pró prio, donde pela prop.III2.2(ji) existe um ultxafiltro D contendo $[F u\{X\}]$. O ultrafiltro D contem o filtro de Frëchet $F$ e portanto, pela demonstração do corolärio da prop.III2.2, D é não principal 
$\operatorname{m~}_{\mathrm{P}} \mathrm{A}_{\mathrm{p}}^{\prime} / \mathrm{D}=\mathrm{A}$ e portanto pelo teorema de tos e pelas proprieda des dos ultrafiltros $I(A) \in D$.

O fato que $I(A) \in D$ e $X=P-I(A) \in D$ contradiz a defini ção de ultrafiltro, logo $x$ deve ser finito e está demonstado o corolärio.

Dado um inteiro positivo d pode-se enunciar na lingua gem de $1 \mathrm{a}$. ordem para corpos valorados uma sentença $\mathrm{A}_{\mathrm{d}}$ ta1 que um corpo $K$ satisfaz esta sentença se e sö se todo polinômio homogêneo de grau $\mathrm{d}$, com coeficientes em $\mathrm{K}$, em $\mathrm{d}^{2}+1$ variảveis admite uma raiz não trivial em $K$. E claro que um corpo satisfaz esta sentença se e sô se ele é $C_{2}(d)$ (pois pode-se anular as $\mathrm{n}-\left(\mathrm{d}^{2}+1\right)$ variáveis restantes de um polinô mio homogêneo de $\mathrm{gr}$ au $\mathrm{d}$ em $n>\mathrm{d}^{2}$ variäveis, para cair em um polinômio de grau d em $\mathrm{d}^{2}+1$ variäveis). Aplicando o coro lärio precedente à sentença $A_{d}$, e usando o fato que $<\mathbb{Z}_{\mathrm{p}}((\mathrm{X}))$, ord, $\mathbb{Z}>\quad \overrightarrow{\mathrm{e}} \mathrm{C}_{2}(\mathrm{~d})$ para todo inteiro positivo d, obtemos o seguinte

COROLARIO 2. - Seja d um inteiro positivo. Então o conjunto I (d) dos primos $p$ tais que $\left\langle Q_{p}, v_{p}, \mathbb{Z}\right\rangle$ não $\vec{e} C_{2}(d)$ éfinito. 


\section{BIBLIOGRAFIA}

[A.K] Ax, J. and Kochen, S., Diophantine problems over local fields I, II. Am. Journ. of Math., 87 (1965), pp.605648.

[C.K] Chang, C.C. and Keiseer, H.J., Mode1 Theory. North-Holland, 1973.

[End] Enderton, H. A Mathematical Introduction to Logic. Academic Press, New York, 1972.

[Gr] Greenberg, M., Lectures on forms in many variables. Benjamin, 1969.

[Kp] Kaplansky, I., Maximal fields with valuations. Duke Math. journ., vol.9, (1942), pg.303-321.

[Ko] Kochen, S., The model theory of local fields. Logic Conf. Kie1 1974.Lect.Notes in Math.499, Ber1in, Springer-Verlag 75.

[Kop] Kopperman, ., Model Theory and its Applications. Boston, A11yn and Bacon, 1972.

[Lg] Lang, S., Some theorems and conjectures in diophantine equations. Bul1. Am. Math. Soc., vol.66, (1960), pg. 240-249.

[Rb] Ribenboim,P., Thëorie des Valuations, Sëminaire de Mathëmatiques Supérieures, Montréa1, 1964

[Sch] Schilling,O.F.G., The theory of valuations, AMS Mathematical Survey, 1950, New York

[ZJ] Zimbarg,J., Introdução à Iógica matemätica, 9o colóquio Bras, de Matemática, Poços de Caldas, 1973. 